\title{
Signatures of Relativistic Neutrinos in CMB Anisotropy and Matter Clustering
}

\author{
Sergei Bashinsky* and Uroš Seljak ${ }^{\dagger}$ \\ Department of Physics, Princeton University, Princeton, NJ 08544
}

(Dated: January 15, 2004)

\begin{abstract}
We present a detailed analytical study of ultra-relativistic neutrinos in cosmological perturbation theory and of the observable signatures of inhomogeneities in the cosmic neutrino background. We note that a modification of perturbation variables that removes all the time derivatives of scalar gravitational potentials from the dynamical equations simplifies their solution notably. The used perturbations of particle number per coordinate, not proper, volume are generally constant on superhorizon scales. In real space an analytical analysis can be extended beyond fluids to neutrinos.

The faster cosmological expansion due to the neutrino background changes the acoustic and damping angular scales of the cosmic microwave background (CMB). But we find that equivalent changes can be produced by varying other standard parameters, including the primordial helium abundance. The low-l integrated Sachs-Wolfe effect is also not sensitive to neutrinos. However, the gravity of neutrino perturbations suppresses the CMB acoustic peaks for the multipoles with $l \gtrsim 200$ while it enhances the amplitude of matter fluctuations on these scales. In addition, the perturbations of relativistic neutrinos generate a unique phase shift of the CMB acoustic oscillations that for adiabatic initial conditions cannot be caused by any other standard physics. The origin of the shift is traced to neutrino free-streaming velocity exceeding the sound speed of the photonbaryon plasma. We find that from a high resolution, low noise instrument such as CMBPOL the effective number of light neutrino species can be determined with an accuracy of $\sigma\left(N_{\nu}\right) \simeq 0.05$ to 0.09, depending on the constraints on the helium abundance.
\end{abstract}

\section{INTRODUCTION}

Neutrinos play a significant role in the evolution of the early universe. They are expected to provide around $40 \%$, e.g. Ref. [1], of the total energy density during the radiation era. The gravitational potentials (metric perturbations) induced by inhomogeneities in the photon and neutrino backgrounds are comparable. Due to the internal photon and neutrino dynamics the potentials decay when the growing acoustic, for photons, or particle, for neutrinos, horizon of the universe becomes of the order of the perturbation scale, i.e. as the perturbation modes "enter the horizon". This decay, in contrast to the constancy of the potential generated during matter domination by freely collapsing cold dark matter (CDM), leads to substantial difference between the amplitude of the acoustic oscillations in the cosmic microwave background (CMB) on the scales that enter the horizon before and after the matter-radiation equality. For example, in the model without neutrinos the amplitude generated by equal primordial power on the smaller scales is 5 times larger, Ref. [2]. In addition, the gravity of both the photon and neutrino perturbations at the horizon entry boosts CDM peculiar velocities, contributing to matter clustering.

Neutrino contribution to the radiation energy density reduces the redshift of the transition from radiation to matter domination, bringing the transition closer to

\footnotetext{
*Electronic address: sergei@princeton.edu

$\dagger$ Electronic address: useljak@princeton.edu
}

CMB decoupling. This too leads to important consequences to both $\mathrm{CMB}$ anisotropies and matter clustering. The reasons are the larger amplitude of the acoustic oscillations entering the horizon in the radiation universe, larger early-ISW effect from the transition proximity, and suppressed growth of matter fluctuations in the radiation epoch. But these and other discussed later effects caused by neutrino background speeding up the cosmological expansion can generally be mimicked by variations of other standard cosmological parameters. For example, the redshift of the radiation-matter equality could be reduced not by neutrino background but by CDM density being smaller than derived from the fits assuming the standard neutrino content.

The internal dynamics of neutrino perturbations bears almost no resemblance to the more familiar acoustic physics of the photon-baryon fluid. All the three main distinctions below arise from neutrinos being fully decoupled and free-streaming since a very early redshift $z_{\nu \text { dec }} \sim 10^{10}$, long before the hydrogen recombination and $\mathrm{CMB}$ photon decoupling at $z_{\gamma \text { dec }} \simeq 1090$.

First, the tightly coupled photon-electron-baryon fluid supports compressional acoustic waves. These waves are little attenuated until the recombination. Neutrino perturbations propagate differently, by means of free streaming. Neutrinos escape overdense regions in every direction; the projection of their velocity on the density gradient spans the entire interval $[-1,1]$ (in units $c=1$.) The dispersion of the perturbation transfer velocity along the density gradient, called "directional dispersion" [3], damps subhorizon neutrino perturbations inversely proportional to time. This damping was noted three decades ago [4]. But it was quickly realized [5] that, regardless 
of their evolution, the subhorizon neutrino perturbations exert negligible gravitational effects on other species.

Second, neutrino stress is locally anisotropic. According to the Einstein's equations, the stress sources the perturbations of space-time metric. The anisotropic stress leads to richer structure of the metric perturbations than locally isotropic fluids can provide.

Third, neutrino perturbations propagate with the speed of light, exceeding the sound speed of acoustic perturbations in the photon-baryon fluid. As a result, the gravitational effect of neutrino perturbations on CMB, viewed in real space, extends beyond the acoustic horizon of primordial inhomogeneities. We find that this leads to a unique phase shift of the CMB mode oscillations in the presence of neutrino gravity.

What new physics can be revealed by the imprint of neutrino gravity on the more easily observable species, such as CMB photons or non-relativistic matter? The considered neutrino signatures probe the ratio of neutrino and photon energy densities, evaluated when the observed scales enter the horizon. Complimentary constraints on the universe composition in the radiation era are set by the predictions of Big Bang nucleosynthesis (BBN) for the primordial abundances of light elements. The baryon to photon ratios inferred from BBN and $\mathrm{CMB}$ are in good agreement with each other, but the presently low observational estimates of the primordial ${ }^{4} \mathrm{He}$ abundance [6-10] favor the effective number of neutrinos, $N_{\nu}$, at BBN below its standard value 3.04. Joint analyses $[11,12]$ of the current data on the primordial ${ }^{4} \mathrm{He}$ and $\mathrm{D} / \mathrm{H}$ abundances and of the cosmological constraints considered by WMAP team [13] (CMB + LSS + Lyman $\alpha$, fit by $\Lambda$ CDM model) give the $2 \sigma$ limits $1.7<N_{\nu}<3.0$. If a neutrino chemical potential, characterizing $\nu / \bar{\nu}$ asymmetries ${ }^{1}$, is treated as a free parameter to be marginalized over, the limits relax [18] to $-1.7<N_{\nu}<4.1$. However, the constraints from BBN and CMB should be combined with caution.

One of the reasons is that the probed redshifts are separated by many orders of magnitude. The processes that determine the BBN yield of light elements extend from the freeze-out of $\nu_{\mu}, \nu_{\tau}$, and (shortly after) $\nu_{e}$ interactions at $z_{\nu \text { dec }} \sim 10^{10}$ to the fusion of light nuclei at $z_{\mathrm{ns}} \sim 4 \times 10^{8}$. On the other hand, the CMB multipoles up to $l \sim 3000$ probe the neutrino density in the redshift range from $z_{l \text { entry }} \sim 6 \times 10^{4}$ to $z_{\text {eq }} \simeq 3.2 \times 10^{3}$ (assuming the "standard" cosmological parameters $[13,19]$.) Either the photon entropy or the number of uncoupled relativistic species per comoving volume may change ${ }^{2}$ in the considerable span of the universe history from BBN

\footnotetext{
1 Any initial differences among the individual $\nu / \bar{\nu}$ asymmetries for the 3 generations of active neutrinos are equilibrated by neutrino oscillations by the time BBN begins, Refs. [14-17].

2 The change of the photon entropy density is tightly constrained below $z_{\gamma \text { chem eq }} \simeq(2-5) \times 10^{6}$, Refs. [20-22], by the Planckian shape of the CMB spectrum from COBE [23]. Although energy
}

to the redshifts probed by CMB. The responsible physical mechanisms could be, though are not limited to, heating from decays of massive particles or fields, e.g. [27-30], such as expected in thermal inflation [31,32], or cooling by thermal contact with other species, Refs. [28, 33].

Another reason is that both $\mathrm{BBN}$ and $\mathrm{CMB}$ constraints depend on certain properties of the uncoupled relativistic species, in addition to their total energy density. For BBN, the relevant characteristics include the asymmetry between the active neutrinos and their antiparticles, their interaction and mixing with other species beyond the standard model, and the cosmological expansion rate, which may be affected by the density of other uncoupled relativistic particles - "dark radiation" (right-handed neutrinos, Goldstone bosons, moduli, etc.) and by more exotic phenomena such as early quintessence, non-minimally coupled fields, or extradimensional physics. On the other hand, the CMB anisotropies and matter clustering do not discriminate between active neutrinos, their antiparticles, and other relativistic degrees of freedom. But the dynamics of cosmological perturbations in the unseen relativistic background becomes important. In this paper we focus on the signatures caused by ultra-relativistic decoupled particles. Their energy spectrum need not be thermal. Given initial conditions, their gravitational impact on the "visible" species indeed depends only on their combined energy density, parameterized by the effective number of neutrino species $N_{\nu}$, eq. (45).

While the impact of neutrinos on the light element production at $\mathrm{BBN}$ has been studied in detail, the neutrino features in the CMB spectra are less well established. Their comprehensive analysis and the investigation of their potential for probing the primordial radiation of non-electromagnetic origin are presented in this paper. The motivations for exploring these features and the related constraints independently from the BBN physics include: verification of the "standard BBN" model (SBBN); guidance in resolving the tensions between SBBN predictions and observational estimates of the light element abundances (the tension presently exists for ${ }^{4} \mathrm{He}$ but in future is also conceivable for other elements as potentially more sensitive experiments with less studied systematics appear); probing the parameter space of extended BBN models in the directions

released into the photon gas at smaller redshifts can still be redistributed among the photons by Compton scattering, the photon production rates, from double Compton scattering $(e \gamma \rightarrow e \gamma \gamma)$ and bremsstrahlung $(e N \rightarrow e N \gamma$ ), become insufficient to change the photon number to its new equilibrium value. This would lead to a Bose-Einstein CMB frequency spectrum with a nonzero chemical potential; see reviews in Refs. [24, 25]. The present agreement between the BBN- and CMB-derived ratio of baryons to photons is an additional evidence against a large change of the comoving photon entropy density. Of course, the above considerations do not limit the change of the energy of the uncoupled relativistic species; see e.g. Ref. [26] for specific scenarios. 
of degeneracies (e.g. the degeneracy in the $\nu$ chemical potential $-N_{\nu}$ plane [18]); constraining the models of high energy physics, frequently leading to decoupled relics, non-standard $\mathrm{BBN}$, particle decays during or after $\mathrm{BBN}$, or modified cosmological expansion; finally, clarifying robustness of the constraints derived from $\mathrm{CMB}$ anisotropies and matter clustering.

The possibility of identifying the background of decoupled ultra-relativistic species with CMB, sometimes complemented by other cosmological probes, has been analyzed extensively $[12,34-39]$ in the past using numerical calculations with Boltzmann integrator codes, such as CMBFAST or CAMB/CosmoMC [40-42], or with simpler codes in that Boltzmann hierarchy is truncated at the quadrupole order in a way that mimics free steaming $[43,44]$. Some of this work forecasted future constraints on the density of relativistic species using likelihood (Fisher matrix) analysis of specific experiments.

The authors of Refs. [2, 45] noted that the CMB modes entering the horizon in the radiation era are perturbed less and the CDM modes more in a model with a larger neutrino to photon ratio. Later work [44] stressed the essential role of neutrino perturbations in breaking the degeneracies between $N_{\nu}$ and the density of non-relativistic matter, set by $\omega_{m} \equiv \Omega_{m} h^{2}$, either of which affects the redshift of radiation-matter equality $z_{\text {eq }}+1=\rho_{m, 0} /\left(\rho_{\gamma, 0}+\rho_{\nu, 0}\right)$. Degeneracies between the variation of neutrino density and of other cosmological parameters were studied numerically in Ref. [34]. This work pointed out that, with a fixed $z_{\text {eq }}$ and fixed angular size of the acoustic CMB horizon, the remaining CMB spectrum variation with $N_{\nu}$ to the third acoustic peak can be practically removed by a same sign change in the scalar spectral index $n_{s}$, and that the matter power spectrum breaks this degeneracy. However, because of the normalization of the calculational results by the height of the first acoustic peak, the neutrino-induced suppression of CMB anisotropy on small scales was explained as increased ISW contribution on large scales. This interpretation propagated into several later papers. We argue at the end of Sec. III and Sec. V A that this interpretation is incorrect.

The Fisher matrix likelihood analysis of Ref. [34] showed that, prior to WMAP, $N_{\nu}$ could not be constrained by $\mathrm{CMB}$ alone. With the WMAP data [46] new analyses $[12,35,38]$ set the upper limits $N_{\nu} \lesssim 7$ or somewhat better if matter clustering or HST data are included. Ref. [38] reported a lower limit $N_{\nu}>1.6$ with $95 \%$ confidence level from WMAP only and $N_{\nu}>1.9$ with HST data added. We find that these constraints can be improved dramatically with the future experiments and become comparable and tighter than those presently derived using the standard BBN model from the primordial element abundances.

Recently, Ref. [47] considered the interaction of neutrino perturbations with tensor gravitational waves. The problem was reduced to an integro-differential equation using the so-called line-of-sight solution for free streaming particles, derived previously in a context of photons [4850]. Numerical integration of this equation showed that neutrinos suppress the amplitude of the gravitational waves entering the horizon in the radiation era and of the related $B$-mode of CMB polarization by about $20 \%$. Even on the largest angular scales the neutrino damping of the tensor correlation functions is predicted to be close to $10 \%$.

In this work we focus on the more significant and, as of now, the only accessible to observations scalar ${ }^{3}$ perturbations. We use an analytic approach. It provides the physical insight into the cosmological role of neutrinos and helps find a quantitatively small but unique signature of neutrino perturbations, the phase shift, which turns out to play the primary role in measuring the neutrino background density with $\mathrm{CMB}$ experiments. The analytical methods developed in this paper are easily applicable to the tensor sector and give results consistent with Ref. [47].

A real-space view of cosmological perturbation dynamics will be essential for obtaining analytic results for neutrino perturbations, which can not be modeled by a fluid. Many equations governing perturbation dynamics in the radiation era are integrated trivially in real space. This permits analytic calculations that would seem hopeless in momentum space. A real space analysis of cosmological perturbations was attempted earlier in Ref. [51] and applied to CMB anisotropy in Refs. [52, 53]. We follow the plane wave formalism developed in Refs. [54-56].

The rest of the paper is organized as follows. In Sec. II we introduce a slight modification to the classical definition of cosmological perturbation variables. A consequence is substantial simplification of the evolution equations, both for their later solution and conceptual understanding. In Sec. III we set up the notations and evolution equations for the radiation-matter universe around the time of $\mathrm{CMB}$ decoupling. Then we study the impact of neutrinos on the evolution of superhorizon perturbations. In Sec. IV we review the Green's function formalism and apply it to find how neutrino perturbations influence the CMB and CDM modes that enter the horizon in the radiation era. A reader not interested in the specifics of the analytic calculations can look at their results in the figures of Secs. III and IV and proceed toward the discussion of Sec. V. In Sec. V we analyse the neutrino signatures in the $\mathrm{CMB}$ and matter perturbation spectra and either their robustness or degeneracy to the variation of other cosmological parameters. In Sec. VI we estimate the accuracy of constraining the effective number of neutrino species from some planned or proposed CMB experiments. We conclude in Sec. VII.

\footnotetext{
3 As customary in cosmology, the term "scalar perturbation" denotes the invariance of the perturbation Fourier modes with respect to the little rotational group: the axial rotations that do not change a mode wave vector $\mathbf{k}$.
} 
Appendix A reviews the linear cosmological perturbation theory and summarizes the properties of the used metric gauges. In Appendix B we prove that all the matter or metric Green's functions in the Newtonian or synchronous gauges vanish for growing adiabatic perturbations beyond the particle horizon. Appendix C contains technical calculations for Sec. IV.

All the following formulas imply the metric signature $(-1,1,1,1)$. Greek indices range from 0 to 3 ; Latin from 1 to 3 . Dots denote the derivatives with respect to conformal time $d \tau \equiv d t / a$, where $a$ is the cosmological scale factor. The universe expansion rate with respect to conformal time is denoted by $\mathcal{H} \equiv \dot{a} / a=a H$, where $H(z)$ is the proper Hubble expansion rate.

\section{DYNAMICAL PERTURBATION VARIABLES}

In this paper we use predominantly the conformal Newtonian, later "Newtonian", gauge [57, 58] and parameterize scalar metric perturbations as

$$
d s^{2}=a^{2}(\tau)\left[(-1-2 \Phi) d \tau^{2}+(1-2 \Psi) d \mathbf{r}^{2}\right]
$$

The potential $\Phi$ determines the gravitational acceleration of free-falling objects $\mathrm{g}=-\nabla \Phi$. $\Psi$ characterizes the perturbations of spatial curvature in this gauge. (This choice of potentials agrees with Ref. [58]. It is related to other frequently cited works as: $\Phi_{\text {there }}=\Psi_{\text {here }}, \Psi_{\text {there }}=\Phi_{\text {here }}$ for Refs. $[57,59]$, and $\Psi_{\text {there }}=\Phi_{\text {here }}, \Phi_{\text {there }}=-\Psi_{\text {here }}$ for Refs. $[2,45,60]$.) In the presence of anisotropic stress, provided by neutrino perturbations, the potentials $\Phi$ and $\Psi$ differ from each other.

Occasionally, we invoke the synchronous, spatially flat, comoving, and uniform density gauges. Their definitions and the relations between various metric gauges are summarized in part 3 of Appendix A.

\section{A. Coordinate particle number densities}

It appears very useful to describe perturbations of matter $^{4}$ species in terms of the variables that rate of change does not depend on the time derivatives of other perturbation variables. This is not so, for example, for the usually considered proper energy density enhancement $\delta \rho_{a} / \rho_{a}$ in the Newtonian gauge, nor the proper phase-space density perturbation $\delta f_{a}$, nor the local CMB temperature anisotropy $\delta T(\hat{\mathbf{n}}) / T$, nor the "effective temperature" perturbation $\delta T / T+\Phi$, for all of which the corresponding conservation equations involve $\dot{\Psi}$ or $\dot{\Phi}+\dot{\Psi}$ terms. Via the Poisson equation, eq. (A46) in Appendix A, these terms, which are dominant on horizon

\footnotetext{
4 In Sec. II "matter" refers to all the dynamical degrees of freedom, whether in relativistic or non-relativistic particles or fields, in contrast to non-dynamical scalar metric perturbations.
}

scales, bring the time derivatives of other matter perturbations. This complicates the description of perturbation evolution.

Instead, we characterize density perturbation of species $a$ in the Newtonian gauge by a variable

$$
d_{a} \equiv \frac{\delta n_{a, \mathrm{coo}}}{n_{a, \mathrm{coo}}}=\delta_{a}-3 \Psi
$$

where

$$
\delta_{a} \equiv \frac{\delta n_{a, \mathrm{prop}}}{n_{a, \mathrm{prop}}} \equiv \frac{\delta \rho_{a}}{\rho_{a}+p_{a}}
$$

is the proper particle number overdensity ${ }^{5}$. The latter is related to the energy momentum tensor $T_{a}^{\mu \nu}$ by eq. (A19) in Appendix A. Unless noted otherwise, we suppose that the matter species in any group $a$ do not interact nongravitationally with the species of the other groups; hence $T_{a}^{\mu \nu}$ is well defined and covariantly conserved. Examples of the species groups $a$ are: photon-baryon plasma, neutrinos, or cold dark matter (CDM).

The species mean velocity and anisotropic stress, defined with eqs. (A19), for scalar perturbations can be described by a velocity potential $u_{a}$ as $v_{i a}=-\nabla_{i} u_{a}$ and by an anisotropic stress potential $\pi_{a}$, eqs. (A19-A21). From the energy and momentum conservation equations (A41) and $d_{a}$ definition (2),

$$
\begin{aligned}
& \dot{d}_{a}=\nabla^{2} u_{a}, \\
& \dot{u}_{a}=c_{a}^{2} d_{a}-\chi_{a} u_{a}+\nabla^{2} \pi_{a}+\Phi+3 c_{a}^{2} \Psi,
\end{aligned}
$$

where $^{6} c_{a}^{2} \equiv d p_{a} / d \rho_{a}$ is the "sound speed" and

$$
\chi_{a} \equiv \mathcal{H}\left(1-3 c_{a}^{2}\right)
$$

is the Hubble drag rate for the species a. Eqs. (4) and (5) can be combined into a single second order equation

$$
\ddot{d}_{a}+\chi_{a} \dot{d}_{a}-c_{a}^{2} \nabla^{2} d_{a}-\nabla^{4} \pi_{a}=\nabla^{2}\left(\Phi+3 c_{a}^{2} \Psi\right) .
$$

For the special cases of CDM and tightly coupled photon fluid with negligible baryon density eq. (7) reduces to

$$
\begin{array}{lc}
\ddot{d}_{c}+\mathcal{H} \dot{d}_{c}=\nabla^{2} \Phi & (\mathrm{CDM}) ; \\
\ddot{d}_{\gamma}-\frac{1}{3} \nabla^{2} d_{\gamma}=\nabla^{2}(\Phi+\Psi) & \text { (photon fluid) } .
\end{array}
$$

The variables $d_{a}$ can be interpreted in the Newtonian gauge as the perturbations of the conserved particle number densities with respect to the coordinate differential volume, $d^{3} \mathbf{r}$, rather than the proper volume,

\footnotetext{
${ }^{5}$ If $n$ is the density of any conserved number, its change in a locally inertial frame for a closed volume $V$ equals $d n / n=-d V / V=$ $d \rho /(\rho+p)$, given the energy conservation $d(\rho V)+p d V=0$.

${ }^{6}$ Except for the generalized proof of superhorizon conservations in Sec. II B, we will assume that for all the species $a$ the local pressure $p_{a}$ is uniquely specified by the local energy density $\rho_{a}$. This assumption is general enough to apply to photon-baryon plasma, cold matter, massless or massive neutrinos, and constant vacuum energy. It is not valid for a classical field (quintessence) or modified Hubble expansion (Cardassian energy.) Without this assumption eq. (4) should be replaced by eq. (39).
} 
$a^{3}(1-3 \Psi) d^{3} \mathbf{r}$. The change of these densities with time is determined only by the particle flux into a unit coordinate volume, eq. (4). It does not explicitly depend on the metric evolution, affecting the proper number densities $\delta_{a}$. More formally, $d_{a}$ corresponds to the gauge invariant quantity

$$
d_{a}=\delta_{a}-3 H_{L}+\nabla^{2} \chi
$$

where $H_{L}$ and $\chi$ parameterize the general perturbation of spatial metric $g_{i j}$ as given by eqs. (A25-A26). Therefore, the variables $d_{a}$ coincide with the particle number overdensities $\delta_{a}$ in the gauge where the spatial metric is unperturbed - the "spatially flat" gauge of Sec. A 3. The density perturbations $d_{a}$ are simply related to the "conserved curvature" perturbations of Ref. [61] on the hypersurfaces of uniform energy density of species $a$, $\zeta_{a} \equiv-H_{L}^{(\text {uniform, } a)}$, as $d_{a}=3 \zeta_{a}$.

Likewise, we eliminate $\dot{\Psi}$ from the equations of perturbation dynamics in phase space. For this purpose we define a variable $d f_{a}(\tau, \mathbf{r}, q, \hat{\mathbf{n}})$ :

$$
d f_{a} \equiv \delta f_{a}+q \frac{\partial \bar{f}_{a}}{\partial q} \Psi
$$

where $\delta f_{a}(\tau, \mathbf{r}, q, \hat{\mathbf{n}})$ is the perturbation of the proper phase space distribution for species $a, \bar{f}_{a}(\tau, q)$ is their unperturbed density in phase space, and the particle comoving momentum $\boldsymbol{q}=q \hat{\mathbf{n}}$ is defined in Appendix A by eq. (A53). The linearized Boltzmann equation (A57) in terms of the variable (11) reads

$$
\left(d f_{a}\right)^{\cdot}+\frac{q}{\epsilon} n_{i} \nabla_{i}\left(d f_{a}\right)=q \frac{\partial \bar{f}_{a}}{\partial q} n_{i} \nabla_{i}\left(\frac{q}{\epsilon} \Psi+\frac{\epsilon}{q} \Phi\right) .
$$

Summation over the repeated index $i=1,2,3$ is implied. Having in mind the applications to collisionless particles, we dropped the collision term and the terms involving the time derivatives of $\bar{f}_{a}$ and of $\partial \bar{f}_{a} / \partial q$.

The number of phase-space coordinates in the Boltzmann equation can be reduced by one, Ref. [62], when the mass of the particles is negligible relatively to their average kinetic energy, so that $\epsilon \simeq p$ and the $f_{a}$ perturbations propagate with the same speed, the speed of light, regardless of the particle energy. Defining a function

$$
D_{a}(\tau, \mathbf{r}, \hat{\mathbf{n}}) \equiv \frac{3}{4} \frac{\int q^{2} d q q d f_{a}(\mathbf{r}, q, \hat{\mathbf{n}}, \tau)}{\int q^{2} d q q \bar{f}_{a}(q)},
$$

and integrating both sides of eq. (12) over $q^{3} d q$, we find

$$
\dot{D}_{a}+n_{i} \nabla_{i} D_{a}=-3 n_{i} \nabla_{i}(\Psi+\Phi) .
$$

The variable $D_{a}(\tau, \mathbf{r}, \hat{\mathbf{n}})$ is related to the energy-averaged phase space distribution perturbation of Refs. [48-50] as $F_{a}(\tau, \mathbf{r}, \hat{\mathbf{n}})=(4 / 3) D_{a}+4 \Psi$. If the free-streaming particles had the thermal velocity distribution at their decoupling then the temperature perturbation of the particles moving in a specified direction $\hat{\mathbf{n}}$ is $\delta T_{a}(\tau, \mathbf{r}, \hat{\mathbf{n}}) / T_{a}=$ $D_{a} / 3+\Psi$.
Eq. (14) is formally solved by

$$
\begin{aligned}
D_{a}(\tau, \mathbf{r}, \hat{\mathbf{n}})= & D_{a, \text { in }}(\mathbf{r}-\hat{\mathbf{n}} \tau, \hat{\mathbf{n}})- \\
& -\left.3 n_{i} \nabla_{i} \int_{0}^{\tau} d \tau^{\prime}(\Phi+\Psi)\right|_{\tau^{\prime}, \mathbf{r}-\hat{\mathbf{n}}\left(\tau-\tau^{\prime}\right)} .
\end{aligned}
$$

In the following subsection we will show that for adiabatic initial conditions $D_{a, \text { in }}(\mathbf{r}, \hat{\mathbf{n}})$ is independent of $\hat{\mathbf{n}}$. It will be related to the conserved superhorizon value of the spatial curvature perturbation $\zeta$ in the uniform density gauge (the "Bardeen's curvature" [63]) as

$$
D_{a, \text { in }}(\mathbf{r}, \hat{\mathbf{n}})=-3 \zeta_{\text {in }}(\mathbf{r})
$$

for all $a$.

Any scalar perturbation $D_{a}(\tau, \mathbf{r}, \hat{\mathbf{n}})$ can be described by scalar multipole potentials $\left\{d_{l, a}(\tau, \mathbf{r})\right\}_{l=0,1, \ldots}$ as

$$
D_{a}(\hat{\mathbf{n}})=\sum_{l=0}^{+\infty}(-1)^{l}(2 l+1) P_{l}\left(\frac{n_{i} \nabla_{i}}{\nabla}\right) \nabla^{l} d_{l, a}
$$

where $P_{l}$ are Legendre polynomials. Since $P_{l}(\mu)$ contains only the powers $\mu^{l-2 q}$ with $q=0,1, \ldots\lfloor l / 2\rfloor$, the gradient operator enters the right hand side of eq. (17) only through natural powers of $n_{i} \nabla_{i}$ or $\nabla^{2}$. The potentials $d_{l, a}$ are gauge invariant for $l \geq 2$.

As follows from eqs. (A55, 11, 13),

$$
\delta T_{a \nu}^{\mu}=4 \rho_{a}\left\langle n^{\mu} n_{\nu}\left(\frac{1}{3} D_{a}(\hat{\mathbf{n}})+\Psi\right)\right\rangle_{\hat{\mathbf{n}}}
$$

where $n^{0} \equiv 1, n_{0} \equiv-1, n^{i}=n_{i}$, and \langle\rangle$_{\hat{\mathbf{n}}}$ stands for $\int d^{2} \Omega_{\hat{\mathbf{n}}} / 4 \pi$. Substituting the multipole expansion (17), remembering the definition of the variables $d_{a}, u_{a}$, and $\pi_{a}$, eqs. (2, A19-A21), and using that for the ultrarelativistic particles $p_{a}=\rho_{a} / 3$, we find

$$
d_{0, a}=d_{a}, \quad d_{1, a}=u_{a}, \quad d_{2, a}=\frac{3}{2} \pi_{a} .
$$

$d_{l, a}$ dynamics follows from eqs. $(14,17)$ and the relation $\mu P_{l}(\mu)=\frac{l+1}{2 l+1} P_{l+1}+\frac{l}{2 l+1} P_{l-1}$ :

$\dot{d}_{l, a}=\frac{l}{2 l+1} d_{l-1, a}+\frac{l+1}{2 l+1} \nabla^{2} d_{l+1, a}+\delta_{l 1}(\Phi+\Psi)$.

(The Kronecker symbol $\delta_{l 1}$ in the last term should not to be confused with a density perturbation.) One can write a formal integral solution of these equations by expanding the line-of-sight solution (15) over the spherical harmonics. ${ }^{7}$

$$
\begin{aligned}
& 7 \text { Namely, } \\
& d_{l, a}(\tau, \mathbf{r})=3\left\{-\frac{j_{l}(k \tau)}{k^{l}} \zeta_{\text {in }}(\mathbf{r})+\right.
\end{aligned}
$$


The scalar metric perturbations $\Phi$ and $\Psi$ are determined from the linearized Einstein equations, eqs. (A42A45). In terms of the introduced dynamical variables,

$$
\begin{aligned}
\nabla^{2} \Psi-3 \gamma \Psi & =\gamma(d+3 \mathcal{H} u) \\
\Psi-\Phi & =3 \gamma \pi
\end{aligned}
$$

where $\gamma \equiv 4 \pi G a^{2}(\rho+p)$ and

$$
d=\sum_{a} x_{a} d_{a}, \quad u=\sum_{a} x_{a} u_{a}, \quad \pi=\sum_{a} x_{a} \pi_{a},
$$

where $x_{a} \equiv\left(\rho_{a}+p_{a}\right) /(\rho+p)$. Eqs. (23-24) for $\Psi$ and $\Phi$ are non-dynamical constraint equations. These constraints, however, do not limit one's freedom of setting the initial matter distribution potentials $d_{l, a}$.

The above equations of matter dynamics, e.g. eqs. $(8,9,14)$ for the model with dark matter, photon fluid, and neutrinos, that are complemented by the elliptic gravitational equations (23-24) form a well posed closed system. They are the basis for our subsequent analytical analysis of the perturbation dynamics.

\section{B. Superhorizon conservation of the coordinate number densities}

To illustrate the conservation laws prior to their general derivation, we start from the model where all the scalar dynamical (matter) degrees of freedom can be described by scalar potentials $d_{l, a}$ that satisfy the evolution equations of the form $(4-5,20)$. Below, $\lambda$ is a characteristic comoving scale of perturbation variation in space. For a harmonic mode it can be taken as $\lambda=1 / k$. Since in the subsequent sections we are interested in the universe evolution long after the inflation, we first assume that the comoving Hubble scale $\mathcal{H}^{-1}(\tau)$ grows throughout all the considered time. Then it is natural to choose the zero of time $\tau$ at the formal limit of the equations $\mathcal{H}^{-1} \rightarrow 0$. This choice will be implied whenever we refer to a specific $\tau$ value, in the context of post-inflationary

$$
\left.+\int_{0}^{\tau} d \tilde{\tau} \frac{j_{l}^{\prime}(k \tilde{\tau})}{k^{l-1}}[\Phi(\tilde{\tau}, \mathbf{r})+\Psi(\tilde{\tau}, \mathbf{r})]\right\}_{k^{2} \rightarrow-\nabla^{2}},
$$

where $j_{l}$ are spherical Bessel functions. This equation is obtained from eqs. (15-16) and (17) by noting that for any analytic function $f(\mathbf{r})$

$$
\begin{aligned}
f(\mathbf{r}-\hat{\mathbf{n}} \tau) & =e^{-\tau n_{i} \nabla_{i}} f(\mathbf{r})= \\
& =\sum_{l=0}^{\infty}(-1)^{l}(2 l+1) P_{l}\left(\frac{n_{i} \nabla_{i}}{\nabla}\right) i^{l} j_{l}(-i \nabla \tau) f(\mathbf{r})
\end{aligned}
$$

In eq. (21), the operators $j_{l}(k \tau) / k^{l}$ and $j_{l}^{\prime}(k \tau) / k^{l-1}$, with $k^{2} \rightarrow$ $-\nabla^{2}$, are well defined as their Taylor expansions involve only even powers of $k$, hence, only integer powers of the Laplace operator $\nabla^{2}$. If the perturbations in eq. (21) refer to a single spatial harmonic plane wave with a wave-vector $\mathbf{k}$ then $-\nabla^{2}$ does become $k^{2}$. expansion. All of the above assumptions will be lifted by the end of the section.

We set the initial conditions at a time $\tau_{\text {in }} \ll \lambda$ as $d_{l, a} \sim\left(\tau_{\text {in }}\right)^{l}$. Such initial conditions are natural for growing modes, where perturbations are finite for $\tau \rightarrow 0$. If the global intrinsic curvature $K$ does not dominate the Friedmann expansion and the universe does not inflate $(w>-1 / 3)$ then, by eqs. (A15, A13), $\mathcal{H} \sim 1 / \tau$ and $\gamma \sim 1 / \tau^{2}$. Then for our initial conditions and $\tau \ll \lambda$ we can drop all the $\nabla^{2}$ terms in the evolution equations up to an error $O\left(\tau^{2} / \lambda^{2}\right)$. We thus find

$$
\begin{aligned}
& \dot{d}_{a} \simeq 0 \\
& \dot{u}_{a} \simeq c_{a}^{2}\left(d_{a}+3 \mathcal{H} u_{a}+3 \Psi\right)-\mathcal{H} u_{a}+\Phi, \\
& \dot{d}_{l, a} \simeq \frac{l}{2 l+1} d_{l-1, a} \quad(\text { free stream. } l \geq 2), \\
& d+3 \mathcal{H} u+3 \Psi \simeq 0, \\
& \Psi-\Phi=3 \gamma \pi .
\end{aligned}
$$

The corresponding solutions scale for $\tau_{\text {in }} \ll \tau \ll \lambda$ as $d_{l, a} \sim \tau^{l}$ and $\Phi \sim \Psi \sim 1$. Specifically, the coordinate particle number density perturbations $d_{a}$ are constant, up to $O\left(\tau^{2} / \lambda^{2}\right)$ corrections. Adiabaticity of the initial perturbations has not been assumed for this result.

If the initial conditions are adiabatic $^{8}$, from eq. (A24), the density and velocity perturbations of all the species in the Newtonian gauge are related in the limit $\tau / \lambda \rightarrow 0$, $\lambda=$ const as

$$
\delta_{a}=-3 \mathcal{H} u_{a}=\delta \quad \text { (adiabatic, } \tau / \lambda \rightarrow 0 \text { ), }
$$

where $\delta$ is a same function of $\tau$ and $\mathbf{r}$ for all the species $a$. Also, by eq. (2),

$$
d_{a}=d \quad \text { (adiabatic, } \tau / \lambda \rightarrow 0 \text { ) }
$$

with $d=\delta-3 \Psi$ for all $a$. Then the terms in eq. (27) that are proportional to $c_{a}^{2}$ cancel by eq. (29). Hence, all

\footnotetext{
8 We define a perturbation as "adiabatic" (a curvature perturbation) if in some space-time coordinates all the proper matter distributions or fields and their proper rate of change, all smoothed over an arbitrary comoving scale $\lambda$, appear unperturbed in the limit $\tau / \lambda \rightarrow 0, \lambda=$ const. (Without the assumption $\mathcal{H} \sim 1 / \tau$, the relevant limit is [64] $\mathcal{H}^{-1}(\tau) / \lambda \rightarrow 0, \lambda=$ const.) In this, "superhorizon", limit the space-time metric in the considered coordinates remains perturbed. We prove in Appendix B that for any wave-vector $\mathbf{k}$ there exists a non-decaying perturbation mode with such properties. This mode satisfies the conditions of adiabaticity of Ref. [65] and Ref. [59]: As shown in this subsection, under very mild natural assumptions about the internal matter dynamics, the corresponding comoving curvature perturbation $\mathcal{R}$ is constant beyond the horizon, up to $O\left(\tau^{2} / \lambda^{2}\right)$ corrections. Since after a coordinate change (A23) the proper energy density and pressure of species $a$ appear perturbed as $\delta \rho_{a}=\dot{\rho}_{a} \delta \tau$ and $\delta p_{a}=\dot{p}_{a} \delta \tau$ in the linear order, all the ratios $\delta \rho_{a} / \dot{\rho}_{a}$ and $\delta p_{a} / \dot{p}_{a}$ in any other gauge in the above limit are equal.
} 
the velocity potentials evolve identically on superhorizon scales in the leading and next to the leading orders in $\tau / \lambda$ :

$$
\left.\dot{u}_{a} \simeq-\mathcal{H} u_{a}+\Phi \quad \text { (adiabatic, } \tau \ll \lambda\right) .
$$

The same is true for all $d_{a}=d$, which remain constant by eq. (26). The leading order evolution of the $l \geq 2$ multipoles depends on the internal dynamics of the species. For example, these multipoles vanish identically for perfect fluids but they grow for neutrinos. Nevertheless, the given definition of adiabaticity demands that $d_{l, a} \rightarrow 0$ in the $\tau \rightarrow 0$ limit. This is consistent with our previous observation that, for the "growing mode" initial conditions, eq. (28) yields $d_{l, a} \sim \tau^{l}$.

In addition to the coordinate particle number density perturbation $d$, two other perturbation variables are known to be constant for adiabatic perturbations on superhorizon scales. These are the spatial curvature perturbation in the comoving gauge

$$
\mathcal{R}=\Psi+\mathcal{H} u,
$$

eqs. (A36, A38), Refs. [59, 65-67], and the curvature perturbation in the uniform density gauge $\zeta$, eq. (A39) [63]. (The sign of $\mathcal{R}$ and $\zeta$ in this paper coincides with the sign of the Newtonian potentials. Most of the references use the opposite sign.) Since the scalar metric perturbations are not dynamical but are fixed by matter perturbations with constraint equations, one may expect a simple relation between the conserved matter perturbation $d$ and the metric perturbations $\mathcal{R}$ or $\zeta$. Indeed, the comparison of eq. (2) and eq. (A39) gives immediately that

$$
d=-3 \zeta .
$$

It also follows from eqs. (A40, A17) that

$$
\mathcal{R}=\zeta+O\left(\frac{\tau^{2}}{\lambda^{2}}\right)
$$

Thus, up to $O\left(\tau^{2} / \lambda^{2}\right)$ corrections, for the growing adiabatic perturbations and all the species $a$,

$$
d_{a}(\tau \ll \lambda, \mathbf{r})=-3 \zeta_{\text {in }}(\mathbf{r}) \quad \text { (adiabatic) }
$$

where $\zeta_{\text {in }}(\mathbf{r})$ is the time-independent superhorizon value of the curvature perturbation $\zeta$. Substituting this result in eq. (17) and remembering that for $l \geq 1 d_{l, a} \sim \tau^{l} \rightarrow 0$, we also find

$$
D_{a}(\tau \ll \lambda, \mathbf{r}, \hat{\mathbf{n}})=-3 \zeta_{\text {in }}(\mathbf{r}) \quad \text { (adiabatic) } .
$$

The conservation of $D_{a}(\hat{\mathbf{n}})$ or, for non-interacting particles with non-negligible mass, of $d f_{a}(q, \hat{\mathbf{n}})$ in eq. (11) is also evident from eq. (14) or (12), in which all the terms with gradients can be dropped for superhorizon growing perturbations by the same arguments as before.

Eq. (7) assumed that local pressure of the species is uniquely determined by their local energy density. Without this assumption, for all the mutually non-interacting groups $a$ of matter species or fields, the energy conservation $T_{a ; \mu}^{0 \mu}=0$ gives

$$
\dot{d}_{a}=\nabla^{2} u_{a}+\frac{\dot{\rho}_{a} \delta p_{a}-\dot{p}_{a} \delta \rho_{a}}{\left(\rho_{a}+p_{a}\right)^{2}} .
$$

(We emphasize that $T_{a}^{\mu \nu}$ and all the variables in eq. (39) are defined Appendix A independently of the nature of the species self-interaction.) As previously, $\nabla^{2} u_{a}$ can be dropped for growing superhorizon perturbations. Since energy density and pressure perturbations transform under a gauge transformation (A23) as $\delta \tilde{\rho}_{a}=\delta \rho_{a}+\dot{\rho}_{a} \delta \tau$ and $\delta \tilde{p}_{a}=\delta p_{a}+\dot{p}_{a} \delta \tau$, the additional term on the right hand side of eq. (39) is gauge invariant. If for a considered group of species $a$, which may interact among themselves but do not couple to the other species, the energy density and pressure become homogeneous as $\tau / \lambda \rightarrow 0$ in some coordinate frame, this term is initially zero in any frame. We call such initial conditions for the species $a$ "internally adiabatic". For them, all the right hand side of eq. (39) vanishes and $d_{a}$ is constant beyond the horizon. Generalization of the arguments that follow eq. (41) gives that $d_{a}$ starts changing only in the order $O\left(\tau^{2} / \lambda^{2}\right)$.

If all the species $a$ are perturbed internally adiabatically, which is automatic for single-component perfect fluids, then we showed that all the $d_{a}$ are constant for growing modes beyond the horizon. However, the variables $d_{a}$ need not be equal for different $a$ 's if the overall perturbation is not adiabatic. In this case

$$
\zeta=-\frac{1}{3} d=-\frac{1}{3} \sum_{a} x_{a} d_{a}
$$

in general changes outside of the horizon as the species enthalpy abundances $x_{a}$, eq. (A10), vary during the expansion. This is essentially the curvaton mechanism of Ref. [68], see Ref. [69] for a modern version, converting isocurvature into curvature perturbations.

Most generally, any system with locally interacting matter and Einstein gravity possesses a covariantly conserved energy-momentum tensor $T^{\mu \nu}$. Therefore, the scalar perturbation variables $d=\delta-3 \Psi$ and $u$ are always well defined with eqs. (A19-A20). From the covariant conservation $T^{0 \mu}{ }_{; \mu}=0$,

$$
\dot{d}=\nabla^{2} u+\frac{\dot{\rho} \delta p-\dot{p} \delta \rho}{(\rho+p)^{2}} .
$$

In Appendix B we show that if a growing adiabatic perturbation is initially localized in a spatial region then all the matter and gravitational Newtonian gauge potentials, including $u$, vanish beyond the particle horizon of this region. Then, by the Gauss's theorem, the velocity divergence term in eq. (41) has zero integral over any volume enclosing the particle horizon. For the initial conditions that are adiabatic as defined in footnote 8 , the gauge invariant quantity $\dot{d}_{\text {non-ad }} \equiv(\dot{\rho} \delta p-\dot{p} \delta \rho) /(\rho+p)^{2}$ tends to zero in the limit $\tau / \lambda \rightarrow 0, \lambda=$ const. If, motivated by either the equivalence principle or analyticity of the system dynamics, we accept that the metric perturbations 
generate $\dot{d}_{\text {non-ad }}$ only in $O\left(\tau / \lambda^{2}\right)$ order (in the frame where the matter is initially unperturbed but so in any other frame because of the gauge invariance of $\dot{d}_{\text {non-ad }}$ ) then in the leading and next to the leading orders in $\tau / \lambda$ the variables $d$ and $\zeta=-d / 3$ are constant. So is constant the comoving gauge curvature perturbation $\mathcal{R}$, eq. (36), up to $O\left(\tau^{2} / \lambda^{2}\right)$ deviations.

If a stage of inflation, defined as cosmic expansion with positive acceleration $d^{2} a / d t^{2}$, hence contracting comoving Hubble scale $\mathcal{H}^{-1}(\tau)$, is also considered, the condition $\tau / \lambda \rightarrow 0$ should be replaced by $\mathcal{H}^{-1}(\tau) / \lambda \rightarrow 0$, Ref. [64]. Then this limit and the related definition of adiabaticity in footnote 8 in the inflating universe apply to the future rather than the past.

Finally, what about the universe evolution that begins with an inflationary stage and proceeds to the canonical Big Bang? Ref. [65] defines adiabaticity and considers the conservation laws in the limit $\lambda \rightarrow \infty$. However, the initial conditions for modes with finite wavelengths can be different from those for the infinitely large scales. To accommodate such physically viable possibilities, we define the adiabatic initial conditions for any fixed finite spatial scale $\lambda=1 / k$ on the stage of increasing $\mathcal{H}^{-1}(\tau)$ by formally evolving the perturbation with the post-inflationary equations backward in time to $\mathcal{H}^{-1} \rightarrow 0$. (Similarly, by evolving forward in time to $\mathcal{H}^{-1} \rightarrow 0$ during the inflation.) This approach allows one to quantify the primordial non-adiabaticity (admixture of isocurvature modes), which can be probed by CMB or matter spectra, at any $k$. The lesser the primordial non-adiabaticity and the "tidal" $O\left(\mathcal{H}^{-2} / \lambda^{2}\right)$ dynamical deviations are, the better the proved conservation laws apply.

\section{RADIATION-MATTER UNIVERSE}

If the primordial perturbations are nearly adiabatic, ${ }^{9}$ the inhomogeneities in the neutrino background may affect only those CMB and CDM perturbations that entered the horizon while the radiation fraction of the universe energy was non-negligible. As for the perturbation modes with larger wavelengths, the number density perturbations $d_{a}=-3 \zeta_{\text {in }}$ remain frozen and the higher angular multipoles $d_{l \geq 1, a}$ of the photon and matter distributions negligible until the horizon entry. By the time these modes enter the horizon and the species distributions start evolving, the neutrino energy density perturbations are too small to have a gravitational impact on their evolution.

\footnotetext{
9 The following argument and its conclusion do not apply to isocurvature initial conditions, considered recently for neutrinos in Ref. [70].
}

Thus in much of this work we will be interested in the perturbation dynamics at the redshift $z \gtrsim 10^{3}$. Barring the possibilities of noticeable early quintessence [71] or the Cardassian modification of Friedmann expansion $[72,73]$, the background expansion at that time can be described by a flat radiation-matter model. The radiation energy density is provided by CMB photons and neutrinos, which mass becomes dynamically relevant only at $z \lesssim m_{\nu} /\left(3 k T_{\nu, 0}\right) \simeq 200 m_{\nu} /(0.1 \mathrm{eV})$ and is neglected here. (The published WMAP [13] 95\% CL limit on the neutrino masses is $m_{\nu}<0.23 \mathrm{eV}$.) The massive matter consists of cold dark matter, $c$, and baryons, $b$.

We begin from establishing notations convenient for the radiation-matter model. The linearized gravitational equations, e.g. (23-24) or (A42-A45), involve the reduced enthalpy background density $\gamma \equiv 4 \pi G a^{2}(\rho+p)$, eqs. (A11-A13). For baryons and $\mathrm{CDM}$, with $\rho_{a}=$ $\rho_{a, 0} / a^{3}$ and negligible pressure, it equals

$$
\gamma_{b(c)}=\frac{4 \pi G \rho_{b(c), 0}}{a}=\frac{3 H_{\mathrm{ref}}^{2} \omega_{b(c)}}{2 a},
$$

where $H_{\text {ref }} \equiv 100 \mathrm{~km} \mathrm{~s}^{-1} \mathrm{Mpc}^{-1}$ and $\omega_{b(c)} \equiv \Omega_{b(c)} h^{2}$. The present WMAP constraints [13] on $\omega_{b}$ and $\omega_{m} \equiv \omega_{b}+\omega_{c}$, assuming the standard neutrino content, are $\omega_{b}=0.024 \pm$ 0.001 and $\omega_{m}=0.14 \pm 0.02$.

The reduced enthalpy of photons, $\gamma_{\gamma}=(16 / 3) \pi G a^{2} \rho_{\gamma}$, at a given redshift $1 / a$ is fixed by today's CMB temperature $T_{\gamma, 0}=2.725 \pm 0.002 \mathrm{~K}$, Ref. [74], as

$$
\gamma_{\gamma}=\frac{16 \pi G \rho_{\gamma, 0}}{3 a^{2}}=\frac{2 H_{\mathrm{ref}}^{2} \omega_{\gamma}}{a^{2}}
$$

where $\omega_{\gamma} \equiv \Omega_{\gamma} h^{2} \approx 2.47 \times 10^{-5}\left(T_{\gamma, 0} / 2.725 K\right)^{4}$. For neutrinos,

$$
\gamma_{\nu}=\alpha_{\nu} \gamma_{\gamma}, \quad \alpha_{\nu} \equiv \frac{\rho_{\nu}}{\rho_{\gamma}} \equiv \frac{7}{8}\left(\frac{4}{11}\right)^{\frac{4}{3}} N_{\nu} \simeq 0.23 N_{\nu},
$$

where the standard Big Bang Nucleosynthesis predicts $N_{\nu} \approx 3.04$, assuming 3 Standard Model neutrino generations and zero neutrino chemical potential. The effective number of neutrino species $N_{\nu} \neq 3$, first, because neutrinos share some of the energy of $e^{+} e^{-}$, annihilating soon after the neutrino decoupling peak, Ref. [75]. Second, because this energy, most of which heats the photons after the annihilation, is somewhat reduced by finite temperature QED corrections $[76,77]$. The physics of both effects is concisely reviewed in Ref. [78].

Of course, here we allow $N_{\nu}$ to be a free parameter. It characterizes the energy density of all the decoupled ultra-relativistic species at the considered moment, implied being after $e^{+} e^{-}$annihilation but before CMB decoupling,

$$
N_{\nu} \equiv \rho_{\text {rel decoup }} /\left[\frac{7}{8}\left(\frac{4}{11}\right)^{\frac{4}{3}} \rho_{\gamma}\right] .
$$

This parameter may have a non-standard value due to either an unaccounted change of $\rho_{\nu}$ or $\rho_{\gamma}$, or due to the 
density of additional weakly interacting ultra-relativistic species $(X)$. The latter would presumably decouple at very high temperature when the universe contained more relativistic degrees of freedom than during the neutrino decoupling. As the particles such as heavy leptons, hadrons, $W$ and $Z$ bosons, Higgs fields, superparticles, etc. become non-relativistic and annihilate, the entropy shared by the coupled photons, electrons, and neutrinos increases. Since the comoving entropy density of the decoupled species $X$ remains unchanged, their contribution to the parameter (45) may become substantially below unity. A light field carrying $g_{X}$ effective degrees of freedom, with the fermionic ones multiplied by $7 / 8$, that decoupled when the remaining particles in thermal contact had $g\left(T_{<X \text { dec }}\right)$ degrees of freedom contributes to the ratio (45) as

$$
\Delta N_{\nu}=\frac{4}{7} g_{X}\left[\frac{g\left(T_{<X \mathrm{dec}}\right)}{g_{\gamma e \nu}}\right]^{\frac{4}{3}},
$$

were $g_{\gamma e \nu}=43 / 4$. So, for example, a hypothetical neutral Majorana fermion or a scalar Goldstone boson that decoupled when the remaining relativistic degrees of freedom were composed of all the fields of the minimal supersymmetric standard model $\left(g\left(T_{<X \text { dec }}\right)=915 / 4\right)$ would give $\Delta N_{\nu} \simeq 1.7 \times 10^{-2}$ and $\Delta N_{\nu} \simeq 9.7 \times 10^{-3}$ correspondingly.

It is sometimes convenient to use the ratio of comoving time $\tau$ to a characteristic time of the radiation-matter energy equality, and the ratio of scale factor $a$ to its value at the equality:

$$
\bar{\tau} \equiv \frac{\tau}{\tau_{e}}, \quad \bar{a} \equiv \frac{a}{a_{\mathrm{eq}}},
$$

where

$$
\begin{aligned}
a_{\mathrm{eq}} & =\frac{\left(1+\alpha_{\nu}\right) \omega_{\gamma}}{\omega_{m}} \approx \\
& \approx \frac{1}{3.5 \cdot 10^{3}}\left(\frac{1+\alpha_{\nu}}{1.69}\right)\left(\frac{0.3 \times 0.7^{2}}{\Omega_{m} h^{2}}\right),
\end{aligned}
$$

and

$$
\begin{aligned}
\tau_{e} & \equiv \frac{\tau_{\mathrm{eq}}}{2(\sqrt{2}-1)}=\frac{1}{H_{\mathrm{ref}}} \sqrt{\frac{a_{\mathrm{eq}}}{\omega_{m}}} \approx \\
& \approx 130 \mathrm{Mpc} \sqrt{\frac{1+\alpha_{\nu}}{1.69}}\left(\frac{0.3 \times 0.7^{2}}{\Omega_{m} h^{2}}\right) .
\end{aligned}
$$

The Friedmann equation for the radiation-matter universe in terms of the variables $(47)$ reads $(d \bar{a} / d \bar{\tau})^{2}=1+\bar{a}$, yielding

$$
\bar{a}=\bar{\tau}+\frac{1}{4} \bar{\tau}^{2} .
$$

We find it useful to introduce the variable

$$
r \equiv \frac{\bar{\tau}}{\bar{a}}=\frac{1}{1+\frac{1}{4} \bar{\tau}}=\frac{2}{1+\sqrt{1+\bar{a}}} .
$$

Note for reference that $\tau=4 \tau_{e}(1-r) / r, d \tau=-4 \tau_{e} d r / r^{2}$, and $a=4 a_{\mathrm{eq}}(1-r) / r^{2}$. In terms of $r$,

$$
\mathcal{H}=\frac{2-r}{\tau}, \quad \gamma_{\nu}=\frac{2 R_{\nu} r^{2}}{\tau^{2}},
$$

where

$$
R_{\nu} \equiv \frac{\rho_{\nu}}{\rho_{r}}=\frac{\alpha_{\nu}}{1+\alpha_{\nu}}
$$

is the neutrino fraction of the total radiation energy density $\rho_{r}=\rho_{\gamma}+\rho_{\nu} ; R_{\nu} \approx 0.408$ for $N_{\nu}=3.04$. The formulas describing the superhorizon perturbation modes, Sec. III C, become very compact if the mode evolution is parameterized by $r$. For example, see eq. (75) for the well known $k=0$ growing mode of the gravitational potential in the radiation-matter neutrinoless model, Ref. [60]. The radiation and matter domination limits of these formulas are easily read off by setting $r$ to 1 and 0 correspondingly.

\section{A. Perturbations in the radiation era}

When the universe energy density is dominated by photon gas and ultra-relativistic neutrinos, for all of which $w_{a}=c_{a}^{2}=\frac{1}{3}$, then

$$
\mathcal{H}^{(r)}=\frac{1}{\tau}, \quad \gamma^{(r)}=\frac{2}{\tau^{2}} .
$$

In the radiation era $x_{\gamma}^{(r)}=1-R_{\nu}, x_{\nu}^{(r)}=R_{\nu}$, and $x_{a}^{(r)}$ for any non-relativistic species is negligible. The evolution equations (9), (14), (23-24) in this regime become

$$
\begin{aligned}
\ddot{d}_{\gamma}-\frac{1}{3} \nabla^{2} d_{\gamma} & =\nabla^{2}(\Phi+\Psi), \\
\dot{D}_{\nu}+n_{i} \nabla_{i} D_{\nu} & =-3 n_{i} \nabla_{i}(\Psi+\Phi), \\
\tau^{2} \nabla^{2} \Psi-6 \Psi & =2 d+\frac{6}{\tau} u \\
\Phi & =\Psi-\frac{6 R_{\nu} \pi_{\nu}}{\tau^{2}} .
\end{aligned}
$$

On the scales well inside the acoustic horizon, $\lambda \ll$ $\tau / \sqrt{3}$, the gravitational terms on the the right hand side of eqs. $(55,56)$ are negligible. The oscillating photon acoustic modes and the free-streaming neutrinos decouple from each other. However, the phase and the amplitude of the acoustic oscillations is set by the perturbation dynamics during the horizon entry, when the gravity of neutrino perturbations played a significant role. Likewise, the neutrino perturbations affect the dark matter peculiar velocities, evolving according to eq. (8), as the matter received a gravitational boost from the radiation when the fluctuations entered the horizon in the radiation era.

If the neutrino density is negligible $\left(R_{\nu} \rightarrow 0\right)$ then the above equations have compact analytic solutions in 
either Fourier space, eqs. $(110,117,127)$, or real space, eqs. $(109,116,126)$. When the neutrino gravity is appreciable, it appears difficult to track the evolution of the Fourier modes analytically through the horizon entry. But in Sec. IV we succeed with an analytical approach in real space.

\section{B. Perturbations in the matter era}

When massive matter, with pressure and anisotropic stress being negligible comparatively with its energy density, becomes the dominating component then by eqs. (52) and (A13)

$$
\mathcal{H}^{(m)}=\frac{2}{\tau}, \quad \gamma^{(m)}=\frac{6}{\tau^{2}} .
$$

Provided the energy density perturbations are also dominated by massive matter, giving negligible $\pi$, by eq. (24) the gravitational potentials $\Phi$ and $\Psi$ are equal. After baryons decouple from CMB photons at $z_{d} \sim 1090$, we can also take $\delta p \approx 0$. Then by eq. (A44)

$$
\ddot{\Phi}+3 \mathcal{H} \dot{\Phi}=0 \text {. }
$$

The corresponding non-decaying solutions are time independent on all scales. The constant $\Phi$ and $\Psi$ modes that enter the particle horizon after $z_{d}$ are easily related to the epoch-independent primordial curvature perturbation $\zeta_{\text {in }}$. Indeed, by applying eq. (A38) to superhorizon scales, where $\mathcal{R}=\zeta_{\text {in }}$ by eq. (A40), one finds

$$
\Phi^{\left(m, \lambda \gg \mathcal{H}_{z_{d}}^{-1}\right)}=\Psi^{\left(m, \lambda \gg \mathcal{H}_{z_{d}}^{-1}\right)}=\frac{3}{5} \zeta_{\text {in }} .
$$

When $\dot{\Phi}=\dot{\Psi}=0$, we can rewrite eq. (14) for ultrarelativistic neutrinos or for photons after their decoupling as

$$
\dot{\Theta}_{a}^{\mathrm{eff}}+n_{i} \nabla_{i} \Theta_{a}^{\mathrm{eff}}=0
$$

where

$$
\Theta_{a}^{\mathrm{eff}}(\tau, \mathbf{r}, \hat{\mathbf{n}}) \equiv \frac{1}{3} D_{a}+\Phi+\Psi .
$$

(If the free-streaming particles were in local thermal equilibrium at their decoupling then $\Theta_{a}^{\text {eff }}(\tau, \mathbf{r}, \hat{\mathbf{n}})$ is the relative temperature perturbation of the particles propagating in the direction $\hat{\mathbf{n}}$ plus the gravitational redshift correction: $\Theta_{a}^{\mathrm{eff}}=\delta T_{a}(\tau, \mathbf{r}, \hat{\mathbf{n}}) / T_{a}+\Phi$.) For superhorizon adiabatic perturbations in the matter era $\Theta_{a}^{\text {eff }}(\tau \ll \lambda, \mathbf{r}, \hat{\mathbf{n}})=-\zeta_{\text {in }}+\Phi+\Psi=\frac{1}{3} \Phi(\mathbf{r})$, eqs. $(16,72)$. The corresponding, "Sachs-Wolfe" [79], solution of eq. (62) at later time is

$$
\Theta_{a}^{\mathrm{eff}}(\tau, \mathbf{r}, \hat{\mathbf{n}})=\frac{1}{3} \Phi(\mathbf{r}-\hat{\mathbf{n}} \tau) .
$$

The related multipole potentials $d_{l, a}$, eq. (17), for a single Fourier harmonic $\Phi_{\mathbf{k}}(\mathbf{r})=\operatorname{Re}\left(A e^{i \mathbf{k} \cdot \mathbf{r}}\right)$ follow from eqs. $(63,64)$ and eq. $(22)$ in footnote 7 on p. 5 as

$$
d_{a}=\left[\frac{\sin (k \tau)}{k \tau}-6\right] \Phi_{\mathbf{k}}, \quad d_{l \geq 1, a}=\frac{j_{l}(k \tau)}{k^{l}} \Phi_{\mathbf{k}} .
$$

The evolution equations for the perturbations of other species also have simple analytic solutions when the gravitational potentials are time independent. We do not write these solutions here because the linear perturbation dynamics in the matter era has been thoroughly studied in the past, and neutrinos, while relativistic, do not modify it. Of course, the power spectra of perturbations in the matter era are affected by neutrinos through the change of the effective initial conditions for the modes that entered the horizon prior to matter domination. We discuss this modification of CMB and matter power spectra in Sec. V.

\section{Superhorizon Scales}

The $l \geq 2$ multipoles $d_{l, a}$ of the phase space distributions for the non-relativistic CDM and baryons are negligible in the linear regime. They are also small for CMB photons, isotropized by scattering prior to hydrogen recombination. The integral solution for the multipoles of free-streaming particles, eq. (21) in footnote 7 , shows that for neutrinos on superhorizon scales $d_{l, \nu} \sim \tau^{l} \zeta_{\text {in }}$. Particularly, the neutrino anisotropic stress potential $\pi_{\nu}=\frac{2}{3} d_{2, \nu}$ is of the order of $\tau^{2} \zeta_{\text {in }}$. Then, by eqs. $(24,52)$, $\Psi-\Phi=3 \gamma_{\nu} \pi_{\nu} \sim R_{\nu} r^{2} \zeta_{\text {in }}$. Hence, in the radiation era, when $r \rightarrow 1$, the neutrino anisotropic stress leads to splitting the Newtonian gauge potentials $\Phi$ and $\Psi$ even on superhorizon scales, Ref. [57]. The effect disappears in the matter and later eras, after the energy-momentum tensor of neutrinos becomes negligible comparatively to that of non-relativistic species.

In Sec. II B we showed that for superhorizon growing adiabatic perturbations $d_{a}=-3 \zeta_{\text {in }}=$ const for all the matter and radiation species. The superhorizon evolution of the $l \geq 1$ multipoles $d_{l, a}$ and gravity is described by eqs. $(33,28-30)$ of Sec. II B. In that section we also observed that for adiabatic perturbations all the velocity potentials $u_{a}$ are equal, up to $O\left(\tau^{2} / \lambda^{2}\right)$ corrections, to the momentum-averaged velocity potential $u$. Then, by eqs. $(28,19)$,

$$
\dot{\pi}_{\nu}=\frac{4}{15} u \quad(\tau \ll \lambda) .
$$

Combining this with eqs. $(33,29-30,35)$ we find a closed equation

$$
\ddot{\pi}_{\nu}+2 \mathcal{H} \dot{\pi}_{\nu}+\frac{4}{5} \gamma_{\nu} \pi_{\nu}=\frac{4}{15} \zeta_{\text {in }} \quad(\tau \ll \lambda) .
$$

Its non-decaying numerical solution for $N_{\nu}=3$ is plotted in Fig. 1 a) with the solid line. Given the neutrino anisotropic stress potential $\pi_{\nu}$, the superhorizon value of the velocity potential $u$ can be calculated from eq. (66). 

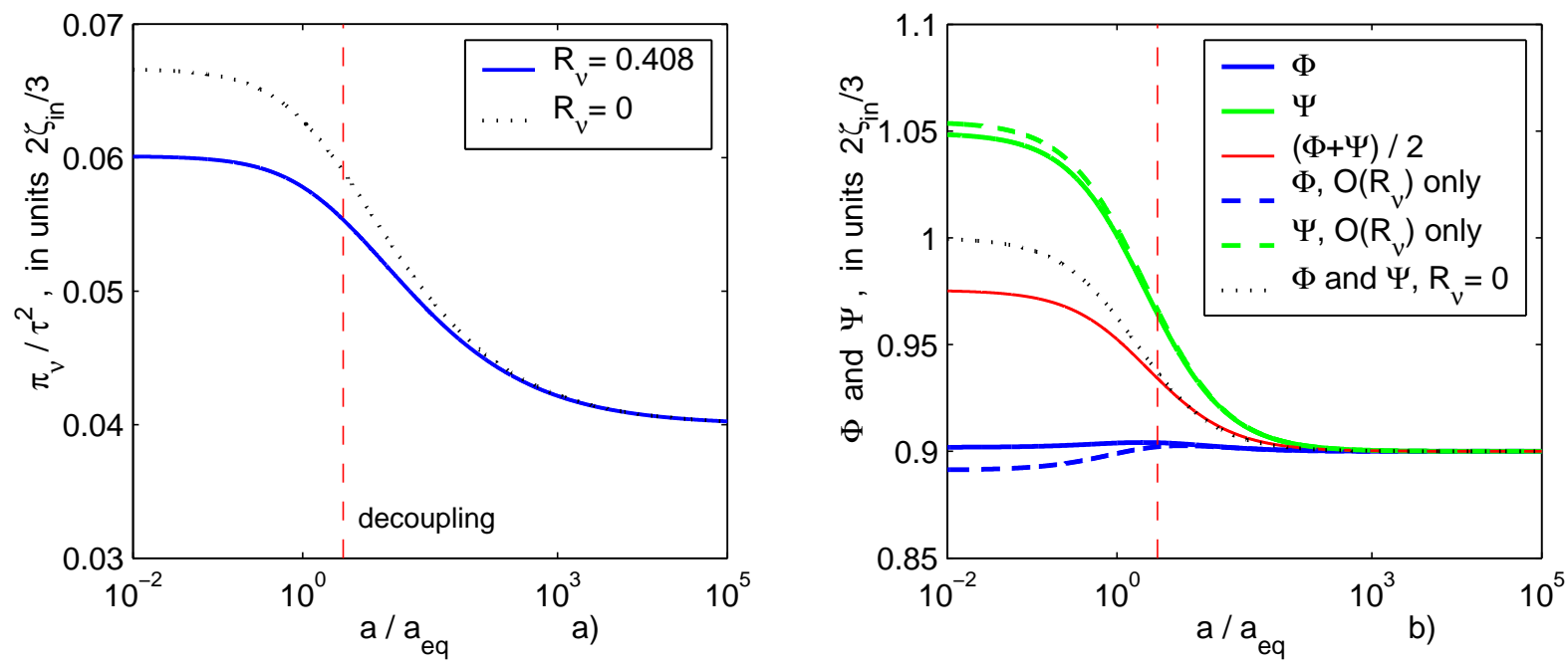

FIG. 1: The evolution of superhorizon adiabatic perturbations in the radiation-matter universe. a) Neutrino anisotropic stress potential. b) The Newtonian gauge gravitational potentials. On both plots, the solid curves show the full result for 3 neutrino species. The dotted curves correspond to 0 neutrino species. The dashed curves on plot b) are the sums of the leading and subleading terms in the expansion of the potentials in $R_{\nu}$, for 3 neutrino species. The dashed vertical lines show $a / a_{\mathrm{eq}}$ at $\mathrm{CMB}$ decoupling, given the cosmological parameters of Ref. [46].

The gravitational potentials $\Phi$ and $\Psi$ are then obtained from eqs. $(33,24)$. The potentials corresponding to the $N_{\nu}=3$ numerical solution of eq. (67) are plotted in Fig. 1 b) with the solid lines.

We observe that, first, coincidentally, the Newtonian potential $\Phi$ is almost unchanged during the radiationmatter transition if $N_{\nu} \approx 3$. Second, when radiation is dynamically significant, the sum $\Phi+\Psi$ is smaller in a universe with a larger effective number of neutrinos. This sum governs the propagation of CMB photons, as seen from eq. (7) with $c_{a}^{2}=\frac{1}{3}$. The following analytical analysis quantifies these observations.

In the radiation era $\mathcal{H}^{(r)}=1 / \tau, \gamma_{\nu}^{(r)}=2 R_{\nu} / \tau^{2}$, and the growing mode solution of eq. (67) is

$$
\pi_{\nu}^{(r, \tau \ll \lambda)}=\frac{\tau^{2}}{15+4 R_{\nu}} \frac{2 \zeta_{\text {in }}}{3} .
$$

Hence, from eqs. $(66,33,24)$,

$$
\begin{aligned}
\Phi^{(r, \tau \ll \lambda)} & =\frac{1}{1+\frac{4}{15} R_{\nu}}\left(\frac{2 \zeta_{\text {in }}}{3}\right), \\
\Psi^{(r, \tau \ll \lambda)} & =\left(1+\frac{2}{5} R_{\nu}\right) \Phi^{(r, \tau \ll \lambda)} .
\end{aligned}
$$

Relation (70) between the potentials in the radiation era was previously derived in Ref. [57].

In the matter era $\mathcal{H}^{(m)}=2 / \tau$ and the $\gamma_{\nu} \pi_{\nu}$ term in eq. (67) is negligible. Then

$$
\pi_{\nu}^{(m, \tau \ll \lambda)}=\frac{\tau^{2}}{25} \frac{2 \zeta_{\text {in }}}{3}
$$

and we obtain the conventional result:

$$
\Phi^{(m, \tau \ll \lambda)}=\Psi^{(m, \tau \ll \lambda)}=\frac{3}{5} \zeta_{\text {in }},
$$

c.f. eq. (61).

In the intermediate regime eq. (67) has no simple exact solution. But the physics of the superhorizon perturbation dynamics in the presence of neutrino anisotropic stress can we studied analytically by expanding the solution in the powers of $R_{\nu}$. The calculations in the zeroth and the first orders are straightforward and are given below.

In the zeroth order, i.e. when the neutrino fraction $R_{\nu}$ is negligible, the gravitational potentials $\Phi$ and $\Psi$ are equal. Then using eqs. $(33,29)$ and remembering that on the superhorizon scales $d=-3 \zeta_{\text {in }}$ we have

$$
\frac{1}{a^{2}}\left(a^{2} u^{\left(R_{\nu} \rightarrow 0\right)}\right)^{\cdot}=\zeta_{\text {in }} .
$$

This relation is easily integrated when $r$ of eq. (51) is taken for the evolution variable:

$$
u^{\left(R_{\nu} \rightarrow 0\right)}=\frac{\tau\left(6+3 r+r^{2}\right)}{30} \zeta_{\text {in }} .
$$

The gravitational potentials then follow from eq. (29) as

$$
\Phi^{\left(R_{\nu} \rightarrow 0\right)}=\Psi^{\left(R_{\nu} \rightarrow 0\right)}=\frac{1}{10}\left(9+\frac{r^{2}}{2}+\frac{r^{3}}{2}\right) \frac{2 \zeta_{\text {in }}}{3} .
$$

This fluid limit solution, known in more lengthy forms before from Ref. [60], is plotted in Fig. $1 \mathrm{~b}$ ) with the dotted line. The anisotropic stress potential $\pi_{\nu}$ of a trace amount of neutrinos can be found by the integration of eqs. $(66,74)$ as

$$
\pi_{\nu}^{\left(R_{\nu} \rightarrow 0\right)}=\frac{\tau^{2} f_{\pi}(r)}{15} \frac{2 \zeta_{\text {in }}}{3},
$$


where

$$
f_{\pi}(r) \equiv \frac{1}{5}\left[\frac{3-r^{2}}{1-r}+\frac{2 r^{2} \ln r}{(1-r)^{2}}\right]
$$

The function $f_{\pi}(r)$ is plotted in Fig. 2 a). Its radiation $(r \rightarrow 1)$ and matter $(r \rightarrow 0)$ era limits are 1 and $\frac{3}{5}$ correspondingly. In Fig. 1 a) we compare the leading order solution $\pi_{\nu}^{\left(R_{\nu} \rightarrow 0\right)} / \tau^{2}$, dotted curve, with the previously found numerical solution for $N_{\nu}=3$.

To determine the $O\left(R_{\nu}\right)$ terms in the gravitational potentials, we rewrite eqs. $(66,67)$ as

$$
\frac{1}{a^{2}}\left(a^{2} u\right)^{\cdot}=\zeta_{\text {in }}-\frac{6 R_{\nu} r^{2}}{\tau^{2}} \pi_{\nu}
$$

where we substituted the result (52) for $\gamma_{\nu}$. The $O\left(R_{\nu}\right)$ solution for the velocity potential is obtained by using the $O(1)$ solution (76) for $\pi_{\nu}$ on the right hand side of eq. (77). After its integration,

$$
u=u^{\left(R_{\nu} \rightarrow 0\right)}-\frac{4 \tau \zeta_{\text {in }}}{45} f_{u} R_{\nu}+O\left(R_{\nu}^{2}\right)
$$

with

$$
f_{u}(r) \equiv \frac{3 r^{2}}{10}\left[5 \frac{(2-r) f_{\pi}-1}{(1-r)}+1-r\right]
$$

Hence, by eqs. (29-30),

$$
\begin{aligned}
& \Phi=\Phi^{\left(R_{\nu} \rightarrow 0\right)}-\frac{8 \zeta_{\text {in }}}{45} f_{\Phi} R_{\nu}+O\left(R_{\nu}^{2}\right) \\
& \Psi=\Phi^{\left(R_{\nu} \rightarrow 0\right)}+\frac{4 \zeta_{\text {in }}}{45} f_{\Psi} R_{\nu}+O\left(R_{\nu}^{2}\right)
\end{aligned}
$$

where

$$
f_{\Psi}(r) \equiv(2-r) f_{u}, \quad f_{\Phi}(r) \equiv \frac{3 r^{2} f_{\pi}-f_{\Psi}}{2} .
$$

All the functions $f_{u}, f_{\Phi}$, and $f_{\Psi}$ tend to 1 in the radiation era limit $r \rightarrow 1$, and to 0 in the matter era limit $r \rightarrow 0$. $f_{\Phi}(r)$ and $f_{\Psi}(r)$ are plotted with solid curves in Fig. 2 b).

The dashed lines in Fig. 1 b) show the sums of the leading and subleading terms in the analytic solutions (79) with $R_{\nu}$ set to its standard value 0.408 , corresponding to $N_{\nu}=3.04$. As seen from the plots, the $O\left(R_{\nu}\right)$ approximations describe the main features of the numerical solutions rather well. The about $11 \%$ smaller than predicted splitting between the potentials $\Psi$ and $\Phi$ in the radiation era corresponds to $4 R_{\nu} / 15 \approx 11 \%$ smaller actual value of the anisotropic stress $\pi_{\nu}$ than it is given by the leading order formula (76), c.f. Fig. 1 a).

By eqs. $(9,14)$, both after the photon decoupling and when the baryon loading is negligible prior to the decoupling, the photon dynamics is affected only by the sum $\Phi+\Psi$. This sum depends on the neutrino abundance as

$$
\frac{\partial(\Phi+\Psi)}{\partial R_{\nu}}=-\frac{4 \zeta_{\text {in }}}{45}\left[2 f_{\Phi}(r)-f_{\Psi}(r)\right]+O\left(R_{\nu}\right) .
$$

The combination $2 f_{\Phi}-f_{\Psi}$ is plotted in Fig. 2 b) with the dash-dotted line. It vanishes at $r \simeq 0.55$, corresponding to $a / a_{\text {eq }} \simeq 6.0$, and has a small negative value at smaller redshifts.

The photon density perturbation $d_{\gamma}$ on superhorizon scales remains constant and independent of $\Phi$ and $\Psi$ evolution. Thus on the scales entering the particle horizon at the redshifts $z \lesssim z_{\text {eq }} / 6$ (for $\omega_{m}=0.14$ and 3 neutrinos, these scales exceed the acoustic horizon at recombination threefold or more) the potential variation and the induced by it ISW effect in the CMB temperature anisotropy are little affected by neutrino perturbations. Even more so, the background and the adiabatic perturbations of relativistic species play no role in the late ISW effect, caused by the global potential decay during the universe transition from matter to dark energy domination. By this time, their energy density is dynamically irrelevant.

\section{STUDYING RADIATION ERA WITH GREEN'S FUNCTIONS}

The evolution of cosmological perturbations in the linear regime may be studied by superposing perturbative solutions (Green's functions) that are localized in real space. It is convenient to consider the Green's functions that vary with only one spatial coordinate [56], say $x$. They are related to the Fourier space perturbation modes by one-dimensional Fourier transformation. For example, for the curvature perturbation $\zeta$,

$$
\zeta(\tau, x)=\int_{-\infty}^{+\infty} \frac{d k}{2 \pi} e^{i k x} \zeta(\tau, k)
$$

Normalizing the Fourier modes to

$$
\zeta(\tau \rightarrow 0, k)=\zeta_{\text {in }},
$$

where $\zeta_{\text {in }}$ is a $k$-independent constant, we see from eq. (81) that

$$
\zeta(\tau \rightarrow 0, x)=\zeta_{\text {in }} \delta_{\mathrm{D}}(x)
$$

$\left(\delta_{\mathrm{D}}(x)\right.$ denotes the Dirac delta function.) Thus the considered Green's function describes the linear evolution of a sheet-like curvature perturbation that was created on the whole plane $x=0$ and is independent of the $y$ and $z$ coordinates.

The initial ratios of the perturbations for different species should be specified as well. In our analysis the initial conditions are assumed adiabatic, but the Green's function method can be generalized to incorporate admixture of isocurvature perturbations. The right hand side of eq. (82) could also be chosen as $A(i k)^{n}$ where $A$ is constant and $n$ is assumed natural. The resulting Green's functions would also be initially localized, with $\lim _{\tau \rightarrow 0} \zeta(\tau, x)=A \delta_{\mathrm{D}}^{(n)}(x)$. These initial conditions are even or odd with respect to the parity transformation $x \rightarrow-x$ if $n$ is even or odd correspondingly. If the initial conditions for the relative species perturbations have 

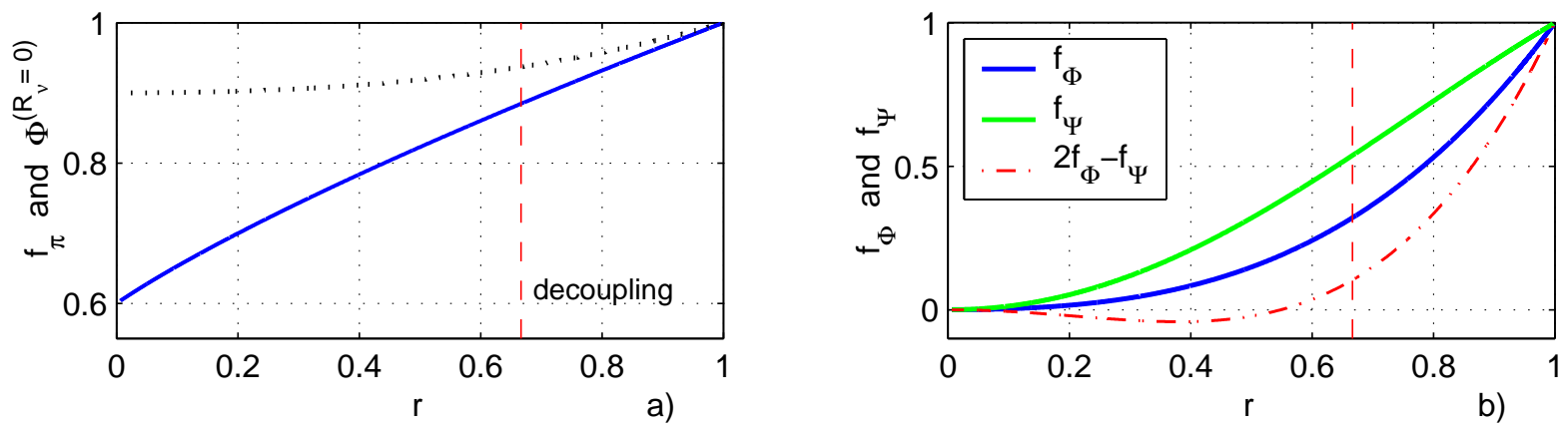

FIG. 2: The functions $f_{\pi}, f_{\Phi}, f_{\Psi}$, and the combination $2 f_{\Phi}-f_{\Psi}$ that appear in the $O\left(R_{\nu}\right)$ order of superhorizon perturbation evolution, as considered in the main text. The evolution variable $r$ is defined by eq. (51). The radiation density domination corresponds to $r \rightarrow 1$, the matter density domination to $r \rightarrow 0$.

the same parity, this parity will be preserved for all $\tau$. It is convenient to impose even initial conditions, as implied by eq. (83), for adiabatic perturbations and odd for isocurvature ones.

We discuss only the "growing" mode Green's functions, corresponding to growing Fourier modes in eq. (81). The decaying solutions of the evolution equations are irrelevant if the primordial perturbations were generated many e-folding before the scales of our interest entered the horizon.

The following two observations prove extremely handy in calculating the Green's function. First, applying the inverse Fourier transformation to eq. (81) and setting $k$ to 0 , one finds the following simple connection between the integral of a Green's function over all the space and the superhorizon Fourier modes of the same variable:

$$
\int_{-\infty}^{+\infty} d x \zeta(\tau, x)=\zeta(\tau, k \rightarrow 0)
$$

or an analogous relation for any other perturbation variable. As it was shown in Sec. II B, for adiabatic perturbations the right hand side of a sum rule such as eq. (84) is time independent for the curvature or density perturbations $\zeta, \mathcal{R}, d_{a}, D_{a}$, or $d f_{a}$. It vanishes for the growing adiabatic perturbations of all the $l>0$ multipole potentials. In the radiation era, the $k \rightarrow 0$ limit for any other perturbation, i.e. involving gravitational potentials, is trivially calculable for any neutrino density from the results of Sec. III C.

Second, the adiabatic Green's functions with even initial conditions (83) identically vanish beyond the particle horizon of the original perturbation, $|x|>\tau$, for all the considered perturbation variables in the Newtonian gauge, including the potentials $\Phi$ and $\Psi$. This result is proven in Appendix B. This is a non-trivial statement, taking into account that the $l \geq 1$ multipole and gravitational potentials are not locally measurable physical quantities and their dynamics is not necessarily causal. ${ }^{10}$

\section{A. Green's functions for phase space distributions}

Neutrinos and decoupled photons are described by their distributions $f_{a}(\tau, \mathbf{r}, q, \hat{\mathbf{n}})$ in the phase space $(\mathbf{r}, \boldsymbol{q} \equiv q \hat{\mathbf{n}}) . \quad$ A scalar perturbation of $f_{a}$ that does not depend on $y$ and $z$ coordinates must also be independent of $n_{y}$ and $n_{z}: \delta f_{a}=\delta f_{a}(\tau, x, q, \mu)$, where $\mu \equiv n_{x}$. For ultra-relativistic free streaming particles, the energyaveraged distribution $D_{a}(\tau, x, \mu)$, eqs. $(13,11)$, satisfies the transport equation

$$
\dot{D}_{a}+\mu \nabla D_{a}=-3 \mu \nabla(\Psi+\Phi),
$$

where $\nabla \equiv \partial / \partial x$, c.f. eq. (14). The corresponding multipole potentials $d_{l, a}(\tau, x)$, eq. (17), equal

$$
(-1)^{l} \nabla^{l} d_{l, a}=\int_{-1}^{1} \frac{d \mu}{2} P_{l}(\mu) D_{a}(\mu) .
$$

To illustrate the application of Green's functions to neutrino dynamics, we first consider the free streaming of massless particles in a time-independent gravitational potential, as it is the case in the matter era, Sec. III B. The transport equation satisfied by the effective temperature Green's function $\Theta_{a}^{\mathrm{eff}}(\tau, x, \mu) \equiv \frac{1}{3} D_{a}+\Phi+\Psi$, eq. (62),

${ }^{10}$ For gravitationally interacting perfect fluids the linear evolution of the Newtonian gravitational potentials turns out to be causal, as it can be shown by generalizing the formalism of Ref. [56] to arbitrary fluids. This is not true in general. As a simple counterexample, consider an absolutely inelastic collision at $x=0$ of two identical sheets of ultra-relativistic particles that are orthogonal to the $x$ axis and move toward each other with opposite velocities. At the moment of the collision the system anisotropic stress at $x=0$ disappears. This changes $\Psi-\Phi$ instantly throughout all the space. 
becomes

$$
\dot{\Theta}_{a}^{\mathrm{eff}}+\mu \nabla \Theta_{a}^{\mathrm{eff}}=0
$$

Given the initial conditions $\Theta_{a}^{\mathrm{eff}}(0, x, \mu)=\Theta_{\text {in }} \delta_{\mathrm{D}}(x)$, it is solved by

$$
\Theta_{a}^{\mathrm{eff}}(\tau, x, \mu)=\Theta_{\mathrm{in}} \delta_{\mathrm{D}}(x-\mu \tau)
$$

The multipole potentials $d_{l, a}$ corresponding to $D_{a}=$ $3\left(\Theta_{a}^{\mathrm{eff}}-\Phi-\Psi\right)$ follow immediately from eq. (86). Remembering the definition of the Legendre polynomials

$$
P_{l}(\mu) \equiv \frac{1}{2^{l} l !} \frac{d^{l}\left(\mu^{2}-1\right)^{l}}{d \mu^{l}},
$$

we find

$d_{l, a}=\frac{3 \Theta_{\mathrm{in}} \tau^{l-1}}{2^{l+1} l !}\left[1-\left(\frac{x}{\tau}\right)^{2}\right]^{l} \theta(\tau-|x|)-3 \delta_{l 0}(\Phi+\Psi)$,

where $\theta$ is the Heaviside step function. In the matter era $\Phi=\Psi=\Phi_{\text {in }} \delta_{\mathrm{D}}(x), \Theta_{\text {in }}=\Phi_{\text {in }} / 3$, and the Fourier transform of the above formula reproduces the modes of eq. (65).

\section{B. Neutrino distributions}

In the radiation era the evolution equations (55-58) can be converted from partial into ordinary differential equations with respect to a dimensionless variable

$$
\chi \equiv \frac{x}{\tau}
$$

Indeed, the growing modes of such perturbations as $d_{\gamma}$, $D_{\nu}, \Phi$, or $\Psi$ during radiation domination have the form $f(\tau, k)=f(k \tau)$. The corresponding Green's functions, c.f. eq. (81), scale as

$$
\begin{aligned}
& \Phi(\tau, x)=\frac{1}{\tau} \bar{\Phi}(\chi), \quad \Psi(\tau, x)=\frac{1}{\tau} \bar{\Psi}(\chi) \\
& d_{\gamma}(\tau, x)=\frac{1}{\tau} \bar{d}_{\gamma}(\chi), \quad D_{\nu}(\tau, x, \mu)=\frac{1}{\tau} \bar{D}_{\nu}(\chi, \mu) .
\end{aligned}
$$

All the first or second partial derivatives of a function $f(\tau, x)=\bar{f}(\chi) / \tau$ are complete $\chi$ derivatives, which we denote by primes, times some power of $\tau$ :

$$
\begin{array}{ll}
\dot{f}=-(\chi \bar{f})^{\prime} / \tau^{2}, & \nabla f=\bar{f}^{\prime} / \tau^{2}, \\
\ddot{f}=\left(\chi^{2} \bar{f}\right)^{\prime \prime} / \tau^{3}, & \nabla \dot{f}=-(\chi \bar{f})^{\prime \prime} / \tau^{3}, \\
\nabla^{2} f=\bar{f}^{\prime \prime} / \tau^{3} .
\end{array}
$$

The powers of $\tau$ can be canceled out of all the terms in the evolution equations (55-58). For future references, let us note that

$$
f(\tau, k)=\int_{-\infty}^{+\infty} d \chi e^{-i k \tau \chi} \bar{f}(\chi)
$$

We define

$$
\Phi_{+} \equiv \frac{\Phi+\Psi}{2}, \quad \Phi_{-} \equiv \frac{\Psi-\Phi}{2} .
$$

The gravitational potential $\Phi_{-}$is sourced directly by neutrino anisotropic stress as described by eq. (58). On the other hand, the motion of photons in the radiation era and of neutrinos, eqs. $(55,56)$, is affected by $\Phi_{+}$only.

Applying differentiation rules (92) to the neutrino transport formula (85), we obtain an easily integrable equation

$$
\left[(\chi-\mu) \bar{D}_{\nu}\right]^{\prime}=6 \mu \bar{\Phi}_{+}^{\prime}
$$

Since the Green's functions vanish for $|\chi|>1$,

$$
(\chi-\mu) \bar{D}_{\nu}=6 \mu \bar{\Phi}_{+} .
$$

Eq. (96) does not constrain $\bar{D}_{\nu}$ at $\chi=\mu$. It is satisfied by

$$
\bar{D}_{\nu}(\chi, \mu)=p_{\nu}(\mu) \delta_{\mathrm{D}}(\chi-\mu)+\frac{6 \mu}{\chi-\mu} \bar{\Phi}_{+}(\chi)
$$

with any function $p_{\nu}(\mu)$. (Even when $\Phi_{+}$and so the right hand side of eq. (85) are identically zero, there are nonzero $D_{\nu}$ solutions that describe free streaming neutrinos in Minkowski space.)

The function $p_{\nu}(\mu)$ in eq. (97) must be fixed by the initial conditions. Considering $\tau k \rightarrow 0$ limit of relation (93) and remembering eq. (38), for any $|\mu| \leq 1$ we find

$$
\int_{-1}^{1} d \chi \bar{D}_{\nu}(\chi, \mu)=D_{\nu}(\tau k \rightarrow 0, \mu)=-3 \zeta_{\text {in }} .
$$

Substituting solution (97) into the left hand side of the above identity,

$$
p_{\nu}(\mu)=-3 \zeta_{\text {in }}-\int_{-1}^{1} d \chi \frac{6 \mu}{\chi-\mu} \bar{\Phi}_{+}(\chi) .
$$

For the multipoles

$$
\bar{D}_{l, \nu}(\chi) \equiv \int_{-1}^{1} \frac{d \mu}{2} P_{l}(\mu) \bar{D}_{\nu}(\chi, \mu)
$$

eqs. (97, 99) give:

$$
\begin{aligned}
& \bar{D}_{l, \nu}(\chi)=-3\left[\frac{1}{2} \zeta_{\text {in }} P_{l}(\chi)+\right. \\
& \left.+\int_{-1}^{1} d \chi^{\prime} \frac{\bar{\Phi}_{+}\left(\chi^{\prime}\right) \chi P_{l}(\chi)+\bar{\Phi}_{+}(\chi) \chi^{\prime} P_{l}\left(\chi^{\prime}\right)}{\chi^{\prime}-\chi}\right] \theta(1-|\chi|) .
\end{aligned}
$$

In Fig. 3 a) the solid line shows the neutrino density perturbation $\bar{D}_{0, \nu}(\chi)=\bar{d}_{\nu}(\chi)$ that is obtained from this equation and the potential $\bar{\Phi}_{+}$in the limit $R_{\nu} \rightarrow 0$, eq. (109), when the integrals in eq. (100) are easily taken. 


\begin{tabular}{c|c}
$f(x)$ & $\int_{x_{1}}^{x_{2}} d x f(x)$ \\
\hline$\frac{1}{(x-a)^{n+1}}, \quad n=1,2, \ldots$ & $-\left.\frac{1}{n(x-a)^{n}}\right|_{x_{1}} ^{x_{2}}$ \\
$\frac{1}{x-a}$ & $\left.\ln |x-a|\right|_{x_{1}} ^{x_{2}}|x-a|$ \\
$\frac{\ln |x-a|}{x-a}$ & $-\left.\frac{\ln |x-a|+\frac{1}{n}}{n(x-a)^{n}}\right|_{x_{1}} ^{x_{2}}$ \\
& $\left.\frac{1}{2} \ln ^{2}|x-a|\right|_{x_{1}} ^{x_{2}}$
\end{tabular}

TABLE I: The integrals of singular generalized functions. Additional valid formulas are obtained by simultaneously multiplying the expressions on the left and on the right by $\operatorname{sign}(x-a)$.

\section{A note on generalized functions}

The expressions under the integrals in eq. (99) or (100) are singular at $\chi^{\prime}=\chi$. The value of the integrals depends on how the singularity is treated during the integration. Physically, this ambiguity corresponds to resolving the last, divergent, term in eq. (97) outside of an interval $\chi \in\left[\mu-\epsilon_{1}, \mu+\epsilon_{2}\right]$ and approximating the $\bar{D}_{l, \nu}$ structure inside the interval by the first, $\delta$-function term in eq. (97). The most direct approach is to take the integral Cauchy's principal values, implying $\epsilon_{1}=\epsilon_{2} \rightarrow 0$.

Soon we will encounter the integrals of even more divergent expressions, such as $x^{-2}$ or $x^{-2} \ln x$, for that even the Cauchy's principal value does not exist. Nevertheless, we can proceed with their meaningful calculation if all the singular expressions are understood as generalized functions. A detailed mathematical treatment of the latter can be found in Ref. [80]. The physical meaning of these calculations is clarified by the following two theorems. The first one states that any generalized function is a finite order generalized derivative of a continuous function. ${ }^{11}$ The second that differentiation of a generalized function $f(x)$ multiplies its Fourier components $f(k)$ by $i k$. Green's functions can be formally defined as Fourier integrals of perturbation modes, eq. (81). Generalized functions provide a consistent, elegant formalism for their manipulation even when the integrals diverge in the Riemann's sense. One could avoid the divergences by working only with sufficiently smooth potentials of singular real space perturbations, e.g., considering $\Phi$ instead of $\delta \rho \propto \nabla^{2} \Phi$, on small scales. But the use of generalized Green's functions simplifies and streamlines the calculations.

11 To be precise, any finite order derivative of a continuous function $f(x)$ with $|f|$ bounded as $|x| \rightarrow \infty$ by a finite power of $|x|$ defines a generalized function and, conversely, any generalized function can be presented as such a derivative.
Table I gives the definite integrals of several singular functions, interpreted as generalized functions. This table is easily understood by noting that the generalized functions corresponding to the expressions on the left are defined as the derivatives of the less singular expressions on the right. Of course, the generalized integration agrees with the conventional one on any interval on that the Riemann integral exists.

Table II lists the Fourier transforms of singular generalized functions. It is useful to remember that the Fourier image of an even real function is even and real and of an odd real function is odd and imaginary.

\section{Gravitational potentials}

Now we turn to the linearized Einstein equations and solve them consistently with the dynamical equations for the relativistic matter. First, we differentiate eq. (58) twice to obtain

$$
\bar{\Phi}_{-}^{\prime \prime}=2 R_{\nu} \bar{D}_{2, \nu},
$$

where we applied the last of eqs. (19). The perturbation $\bar{D}_{2, \nu}(\chi)$ on the right hand side is given in terms of the potential $\bar{\Phi}_{+}$by eq. (100) with $l=2$.

Second, we note that for adiabatic perturbations in the radiation era $\delta p / \delta \rho=1 / 3$. Then we can easily eliminate all the matter perturbations from Einstein eqs. (A42, A44-A45) to find

$$
\ddot{\Psi}-\frac{2}{3} \nabla^{2} \Psi+\frac{1}{3} \nabla^{2} \Phi+\frac{1}{\tau}(3 \dot{\Psi}+\dot{\Phi})=0 .
$$

Using eqs. (92), we obtain a relation that can be integrated once trivially, giving

$$
\left(\chi^{2}-\frac{1}{3}\right) \bar{\Phi}_{+}^{\prime}+\left(\chi^{2}-1\right) \bar{\Phi}_{-}^{\prime}-2 \chi \bar{\Phi}_{+}=0
$$

The general solution of the above equation is

$$
\begin{gathered}
\frac{\bar{\Phi}_{+}(\chi)}{\chi^{2}-\frac{1}{3}}=\mathrm{const}-F_{-}(\chi) \\
F_{-}(\chi) \equiv \int_{-1}^{\chi} d \chi^{\prime} \frac{\chi^{\prime 2}-1}{\left(\chi^{\prime 2}-\frac{1}{3}\right)^{2}} \bar{\Phi}_{-}^{\prime}\left(\chi^{\prime}\right) .
\end{gathered}
$$

The integration constant on the right hand side of eq. (104) is unambiguously defined for all $\chi$ if only we specify how the integral in the second equation is understood for $\chi>-\frac{1}{\sqrt{3}}$, when the integration path encounters singularities at $\chi^{\prime}= \pm \frac{1}{\sqrt{3}}$. As discussed in the preceding subsection, we treat the singular expression under the integral as a generalized function. Then, given a certain $\bar{\Phi}_{-}^{\prime}$, one can integrate the singular terms using Table I.

The constant in eq. (104) may differ among the $\chi$ intervals $\left(-\infty,-\frac{1}{\sqrt{3}}\right),\left(-\frac{1}{\sqrt{3}}, \frac{1}{\sqrt{3}}\right)$, and $\left(\frac{1}{\sqrt{3}}, \infty\right)$. For example, if $R_{\nu}=0$ then $\bar{\Phi}_{-}=0, F_{-}(\chi)=0$ and $\bar{\Phi}_{+}(\chi)$ vanishes 


$$
\begin{array}{c|c}
\bar{f}(\chi)=\int_{-\infty}^{+\infty} \frac{d \varphi}{2 \pi} e^{i \varphi \chi} f(\varphi) & f(\varphi)=\int_{-\infty}^{+\infty} d \chi e^{-i \varphi \chi} \bar{f}(\chi) \\
\hline \delta^{(n)}(\chi-a), n=0,1,2, \ldots & (i \varphi)^{n} e^{-i a \varphi} \\
\operatorname{sign} \chi & \frac{2}{i \varphi} \\
(\chi-a)^{-n}, n=1,2, \ldots & \frac{(-i)^{n} \pi}{(n-1) !} \varphi^{n-1} \operatorname{sign} \varphi e^{-i a \varphi} \\
\chi^{-n} \operatorname{sign} \chi, n=1,2, \ldots & -\frac{2(-i)^{n-1}}{(n-1) !} \varphi^{n-1}[\ln |\varphi|-\psi(n)] \\
\ln |\chi| & -\pi\left[\frac{1}{|\varphi|}+2 \gamma \delta(\varphi)\right] \\
\chi^{-n} \ln |\chi|, n=1,2, \ldots & -\frac{(-i)^{n} \pi}{(n-1) !} \varphi^{n-1} \operatorname{sign} \varphi[\ln |\varphi|-\psi(n)] \\
\chi^{-n} \operatorname{sign} \chi \ln |\chi|, n=1,2, \ldots & \frac{(-i)^{n-1}}{(n-1) !} \varphi^{n-1}\left\{[\ln |\varphi|-\psi(n)]^{2}-\frac{1}{6} \pi^{2}-\psi^{\prime}(n)\right\}
\end{array}
$$

TABLE II: The Fourier transforms of singular generalized functions. The derivation of these results can be found in Ref. [80]. The values of $\psi(n)$, the logarithmic derivative of the gamma function, for a natural argument follow recursively from $\psi(n+1)=$ $\frac{1}{n}+\psi(n)$ and $\psi(1)=-\gamma$, where $\gamma \simeq 0.5772$ is the Euler's constant. In every case, shifting the transformed function argument by a constant $a$, as displayed in the first and third lines, multiplies the Fourier image by $e^{-i a \varphi}$.

for $|\chi|>\frac{1}{\sqrt{3}}$ but not for $|\chi|<\frac{1}{\sqrt{3}}$, c.f. eq. (109). As shown in Appendix B, with the initial conditions that are adiabatic and satisfy eq. (83), the metric must remain unperturbed beyond the particle horizon $|\chi|=1$. From this we immediately conclude that that const $=0$ in the interval $\left(-\infty,-\frac{1}{\sqrt{3}}\right)$. Taking into account that $F_{-}(\chi \geq 1)=0$ because $\bar{\Phi}_{-}^{\prime}(\chi)$ is odd, we see that the constant also vanishes for $\chi \geq \frac{1}{\sqrt{3}}$. Denoting the value of the constant in the interval $\left(-\frac{1}{\sqrt{3}}, \frac{1}{\sqrt{3}}\right)$ by $p_{\Phi}$, we thus have

$$
\bar{\Phi}_{+}(\chi)=\left(\chi^{2}-\frac{1}{3}\right)\left[p_{\Phi} \theta\left(\frac{1}{\sqrt{3}}-|\chi|\right)-F_{-}(\chi)\right] .
$$

$p_{\Phi}$ may be calculated from the results (69-70) for superhorizon Fourier modes in the radiation era that give

$$
\int_{-1}^{1} d \chi \bar{\Phi}_{+}(\chi)=\frac{1+\frac{1}{5} R_{\nu}}{1+\frac{4}{15} R_{\nu}} \frac{2 \zeta_{\text {in }}}{3}
$$

In the following subsection we obtain another, equivalent but easier to apply, condition fixing $p_{\Phi}$.

The system of the integro-differential equations (101) and (105-106) for the pair $\left(\bar{\Phi}_{+}, \bar{\Phi}_{-}^{\prime}\right)$ can be solved by iterations starting from the solution in the limit $R_{\nu} \rightarrow 0$. The latter is

$$
\begin{aligned}
& \bar{\Phi}_{-}^{\left(R_{\nu} \rightarrow 0\right)}=0, \\
& \bar{\Phi}_{+}^{\left(R_{\nu} \rightarrow 0\right)}=\frac{3 \sqrt{3} \zeta_{\text {in }}}{2}\left(\frac{1}{3}-\chi^{2}\right) \theta\left(\frac{1}{\sqrt{3}}-|\chi|\right),
\end{aligned}
$$

as immediately follows from eq. (101) and eqs. (106107). Note that the Fourier transform (93) of the last Green's function matches the well known potential modes in tightly coupled radiation fluid:

$$
\Phi_{+}^{\left(R_{\nu} \rightarrow 0\right)}(\tau, k)=2 \zeta_{\text {in }}\left(\frac{\sin \varphi_{s}}{\varphi_{s}^{3}}-\frac{\cos \varphi_{s}}{\varphi_{s}^{2}}\right),
$$

with $\varphi_{s}=k \tau / \sqrt{3}$.

The next iteration step, giving the potentials in $O\left(R_{\nu}\right)$ order, is performed in Appendix C. Fig. $3 \mathrm{~b}$ ) shows the zeroth order $\bar{\Phi}_{+}$(dotted) and the $O\left(R_{\nu}\right)$ corrected $\bar{\Phi}_{+}$ (solid) and $\bar{\Phi}_{-}$(dashed) potentials for $N_{\nu}=3.04$.

\section{E. Neutrino effect on CMB perturbations}

The Green's function for the photon density perturbation can be easily found in terms of the gravitational potential $\Phi_{+}$. From eqs. $(55,92)$ we obtain

$$
\left(\chi^{2}-\frac{1}{3}\right) \bar{d}_{\gamma}=2 \bar{\Phi}_{+} .
$$

The general solution of this equation is

$$
\bar{d}_{\gamma}=p_{\gamma} \delta_{\mathrm{D}}\left(|\chi|-\frac{1}{\sqrt{3}}\right)+\frac{2 \bar{\Phi}_{+}}{\chi^{2}-\frac{1}{3}} .
$$

The delta function pre-factor $p_{\gamma}$ is fixed by the condition $\int_{-1}^{1} d \chi \bar{d}_{\gamma}(\chi)=d_{\gamma}(k \tau \rightarrow 0)=-3 \zeta_{\text {in }}$, giving

$$
p_{\gamma}=-\frac{3}{2} \zeta_{\text {in }}-\int_{-1}^{1} d \chi \frac{\bar{\Phi}_{+}(\chi)}{\chi^{2}-\frac{1}{3}} .
$$

The pre-factor $p_{\gamma}$ can be related to the constant $p_{\Phi}$, appearing in eq. (106), by applying the "Poisson law" (57). When eq. (57) is considered as a relation among the Green's functions, the only delta-function singularity 

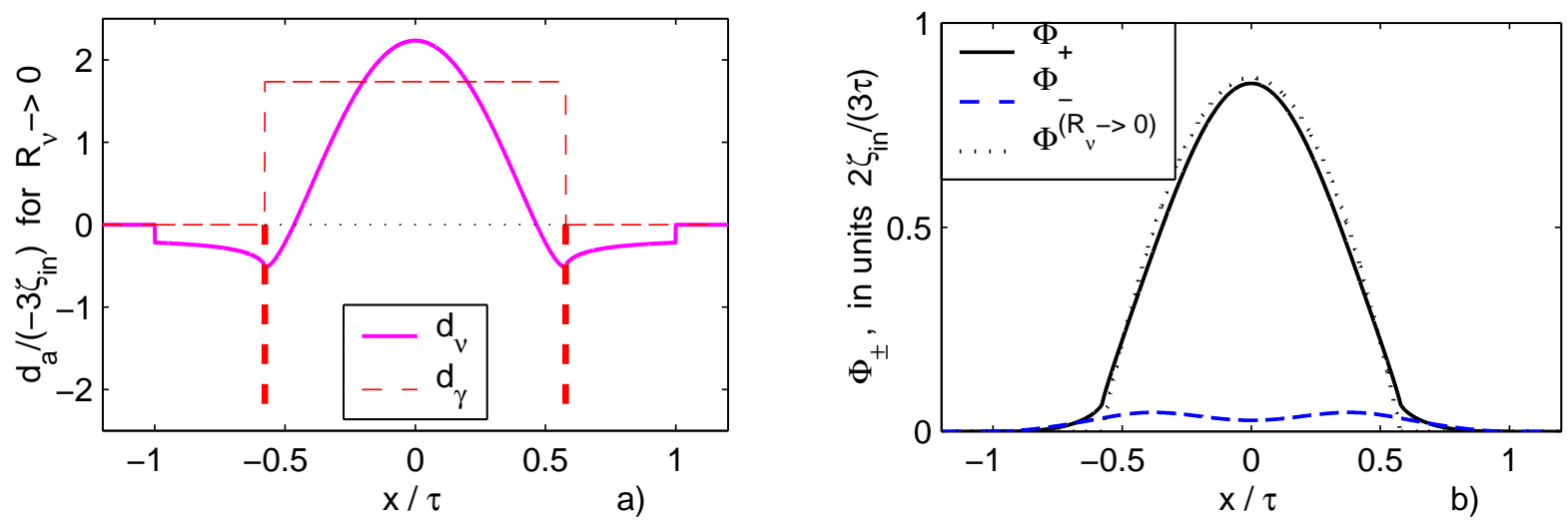

FIG. 3: a) Adiabatic Green's functions for neutrino (solid) and photon (dashed) number density perturbations in the radiation era. The neutrino fraction, $R_{\nu}$, of the radiation density is assumed infinitesimal. b) Adiabatic Green's functions for the gravitational potentials $\Phi_{ \pm} \equiv(\Psi \pm \Phi) / 2$ in the radiation era. The solid and dashed curves are the sums of the $O\left(R_{\nu}^{0}\right)$ and $O\left(R_{\nu}\right)$ terms for three neutrino species. The dotted line is $\Phi_{+}=\Phi$ for $R_{\nu} \rightarrow 0$.

appearing on its right hand side is the one provided by the photon density perturbation (112). As for the left hand side, where $\Psi=\Phi_{+}+\Phi_{-}$, the only deltafunction comes from the double derivative of the term $\left(\chi^{2}-\frac{1}{3}\right) p_{\Phi} \theta\left(\frac{1}{\sqrt{3}}-|\chi|\right)$ in eq. (106). The equality of these contributions requires

$$
p_{\Phi}=-\sqrt{3}\left(1-R_{\nu}\right) p_{\gamma} .
$$

Substituting eq. (106) in (113) and eliminating $p_{\Phi}$ with the relation above, we obtain

$$
p_{\gamma}=\frac{1}{1-2 R_{\nu}}\left[\frac{3}{2} \zeta_{\text {in }}-\int_{-1}^{1} d \chi F_{-}(\chi)\right] .
$$

Calculating $p_{\Phi}$ from the last two equations is somewhat easier than from eq. (107).

Now we have all the analytic tools to analyze how neutrinos affect CMB perturbations. The evolution of metric perturbations without neutrinos is given by eqs. (108109). Then the photon density Green's function follows from eqs. $(112,115)$ as

$$
\begin{aligned}
\bar{d}_{\gamma}^{\left(R_{\nu} \rightarrow 0\right)}=-3 \zeta_{\text {in }} & {\left[\sqrt{3} \theta\left(\frac{1}{\sqrt{3}}-|\chi|\right)-\right.} \\
& \left.-\frac{1}{2} \delta_{\mathrm{D}}\left(|\chi|-\frac{1}{\sqrt{3}}\right)\right] .
\end{aligned}
$$

Its Fourier transform (93) leads to the photon density Fourier modes in the radiation era:

$$
d_{\gamma}^{\left(R_{\nu} \rightarrow 0\right)}(\tau, k)=-3 \zeta_{\text {in }}\left(\frac{2 \sin \varphi_{s}}{\varphi_{s}}-\cos \varphi_{s}\right),
$$

with $\varphi_{s}=k \tau / \sqrt{3}$. In particular, without neutrinos the photon density modes oscillate under the acoustic horizon $\left(\varphi_{s} \gg 1\right)$ as a pure $\varphi_{s}$ cosine.

The predictions for both the phase and the amplitude of the photon mode oscillations differ when the gravity of neutrino perturbations is taken into account. The oscillations of the Fourier modes on subhorizon scales are described by the singular terms in the real space Green's functions. For the photon density (112) these are the $\delta$-function and $\left(\chi \pm \frac{1}{\sqrt{3}}\right)^{-1}$ singularities at $\chi= \pm \frac{1}{\sqrt{3}}$ :

$$
\bar{d}_{\gamma}(\chi)=p_{\gamma} \delta_{\mathrm{D}}\left(|\chi|-\frac{1}{\sqrt{3}}\right)+\frac{2 r_{\gamma}}{\chi^{2}-\frac{1}{3}}+\ldots,
$$

where

$$
r_{\gamma}=\bar{\Phi}_{+}(1 / \sqrt{3})
$$

and the dots stand for more regular terms. The Fourier transform of eq. (118) follows from the first and third lines of Table II, where $n$ is set to 0 and 1 , as

$$
d_{\gamma}(\tau, k)=2\left(p_{\gamma} \cos \varphi_{s}-r_{\gamma} \pi \sqrt{3} \sin \varphi_{s}\right)+O\left(\varphi_{s}^{-1}\right) .
$$

A non-zero phase shift with respect to the $\cos \varphi_{s}$ oscillations is generated whenever $r_{\gamma} \neq 0$. By eq. (119) this can happen for adiabatic perturbations if only some perturbations propagate faster than the sound speed in the photon fluid, and thus are able to generate metric perturbations beyond the acoustic horizon. This is the case for the neutrino perturbations, propagating with the speed of light, Fig. 3 a).

The values of $p_{\gamma}$ and $r_{\gamma}$ in eq. (118) are calculated in $O\left(R_{\nu}\right)$ order in Appendix C. With its results (C6) and (C7), the mode (120) can be presented as

$$
d_{\gamma}(\tau, k)=3 \zeta_{\text {in }}\left(1+\Delta_{\gamma}\right) \cos \left(\varphi_{s}+\delta \varphi\right)+O\left(\varphi_{s}^{-1}\right),
$$

where

$$
\begin{aligned}
\Delta_{\gamma} & \simeq-0.2683 R_{\nu}+O\left(R_{\nu}^{2}\right), \\
\delta \varphi & \simeq 0.1912 \pi R_{\nu}+O\left(R_{\nu}^{2}\right) .
\end{aligned}
$$

As demonstrated in Fig. 4 a), our theoretical predictions are in excellent agreement with numerical calculations 
for the radiation era, at the redshift $z=10^{7}$, obtained with CMBFAST [42]. The numerical calculations show that the $O\left(R_{\nu}^{2}\right)$ corrections contribute to $\Delta_{\gamma}$ and $\delta \varphi$ less than $10 \%$ when $N_{\nu} \sim 3$.

Our analytical results (121-122) can be compared with numerical fit (B7) of Ref. [2], giving for the photon overdensity in the radiation era

$$
d_{\gamma}^{(\text {HS fit })}(\tau, k) \approx \frac{9 \Psi(\tau k \rightarrow 0) \cos \varphi_{s}}{2\left(1+\frac{2}{5} R_{\nu}\right)}=\frac{3 \zeta_{\text {in }} \cos \varphi_{s}}{\left(1+\frac{4}{15} R_{\nu}\right)}
$$

This formula misses the phase shift due to neutrino perturbations but describes the decrease of the oscillation amplitude with the increase of neutrino fraction remarkably well. In the $O\left(R_{\nu}\right)$ order it coincides with the result (122) by better that $1 \%$.

Although the primordial magnitude of the cosmological perturbations is unmeasurable directly, one can detect the amplitude change of the CMB acoustic oscillations, predicted by the first of eqs. (122), by comparing CMB and dark matter density fluctuations. The latter, however, are themselves affected by neutrinos. In the next section we find the leading, $O\left(R_{\nu}\right)$, corrections to the CDM density perturbation modes entering the horizon in the radiation era.

\section{F. Neutrino effect on CDM perturbations}

The evolution of the CDM coordinate density perturbation $d_{c}$ is given by eq. (8). In the radiation era it becomes

$$
\ddot{d}_{c}+\frac{1}{\tau} \dot{d}_{c}=\nabla^{2} \Phi
$$

Using the CDM Green's function ansatz $d_{c}(\tau, x)=$ $\bar{d}_{c}(\chi) / \tau$ and the differentiation rules (92), canceling the common factors $1 / \tau^{3}$, and integrating the resulting equation once trivially, we find

$$
\left(\chi^{2} \bar{d}_{c}\right)^{\prime}-\chi \bar{d}_{c}=\bar{\Phi}^{\prime}
$$

In Appendix $\mathrm{C}$ we show that in the $R_{\nu} \rightarrow 0$ limit and with the adiabatic initial conditions this equation gives for $\chi \neq 0$

$$
\bar{d}_{c}^{\left(R_{\nu} \rightarrow 0\right)}=-3 \zeta_{\text {in }}\left(\sqrt{3}-\frac{1}{|\chi|}\right) \theta\left(\frac{1}{\sqrt{3}}-|\chi|\right) .
$$

In Fourier space

$d_{c}^{\left(R_{\nu} \rightarrow 0\right)}(\tau, k)=-6 \zeta_{\text {in }}\left(\ln \varphi_{s}+\gamma-\frac{1}{2}-\operatorname{ci} \varphi_{s}+\frac{\sin \varphi_{s}}{\varphi_{s}}\right)$,

with $\varphi_{s}=k \tau / \sqrt{3}$.

A finite neutrino fraction of the radiation energy density $R_{\nu}$ affects the gravitational potential on the right hand side of eq. (124) and so the matter density perturbation $d_{c}$. On the scales well inside the acoustic horizon $\left(\varphi_{s} \gg 1\right)$, when the potential term in eq. (124) can be dropped, the general solution for CDM Fourier modes should be of the form

$$
\begin{array}{r}
d_{c}(\tau, k)=-6 \zeta_{\text {in }}\left(1+\Delta_{c}\right)\left(\ln \varphi_{s}+\gamma-\frac{1}{2}+\delta c\right)+ \\
+O\left(\varphi_{s}^{-1}\right)
\end{array}
$$

The values of the integration constants $\Delta_{c}$ and $\delta c$ are determined by the mode dynamics during the horizon entry and are sensitive to the gravity of neutrino perturbations. The real space calculations in Appendix $\mathrm{C}$ give that

$$
\begin{aligned}
\Delta_{c} & \simeq 0.2297 R_{\nu}+O\left(R_{\nu}^{2}\right) \\
\delta c & =-0.6323 R_{\nu}+O\left(R_{\nu}^{2}\right) .
\end{aligned}
$$

The density perturbation $(128-129)$ in $O\left(R_{\nu}\right)$ order is compared with the radiation era $\left(z=10^{7}\right)$ CMBFAST calculations in Fig. $4 \mathrm{~b})$. When $k \tau \gg 1$, the $O\left(R_{\nu}\right)$ analytical results underpredict $d_{c}$ variation for $N_{\nu}$ change from 0 to 1 by $11.6 \%$ and from 0 to 3 by $23 \%$. Since in the second case $R_{\nu}$ changes by 2.1 times more than in the first one, the twofold increase in the relative error is consistent with the origin of this deviation from the $O\left(R_{\nu}^{2}\right)$ corrections.

\section{NEUTRINO SIGNATURES IN CMB AND MATTER SPECTRA}

Decoupled neutrinos affect observable cosmological probes both by the gravity of their perturbations and by the change of the cosmological expansion rate due to the contribution of the neutrino background to the universe energy density. The first effect is prominent when cosmological perturbation modes enter the horizon in the radiation era. The corresponding modifications of the photon and CDM perturbations were found in the previous section. The perturbations remain to be propagated to the later epochs and related to observable statistical power spectra. These tasks are addressed in the current section.

\section{A. CMB power spectra}

Theory overview

While photons are tightly coupled, their number density perturbation $d_{\gamma}$ satisfies the equation

$$
\begin{aligned}
\ddot{d}_{\gamma}+\frac{\mathcal{H} R_{b}}{1+R_{b}} \dot{d}_{\gamma}-c_{s}^{2} \nabla^{2} d_{\gamma} & = \\
& =2 \tau_{d} \nabla^{2} \dot{d}_{\gamma}+\nabla^{2}\left(\Phi+\frac{\Psi}{1+R_{b}}\right)
\end{aligned}
$$



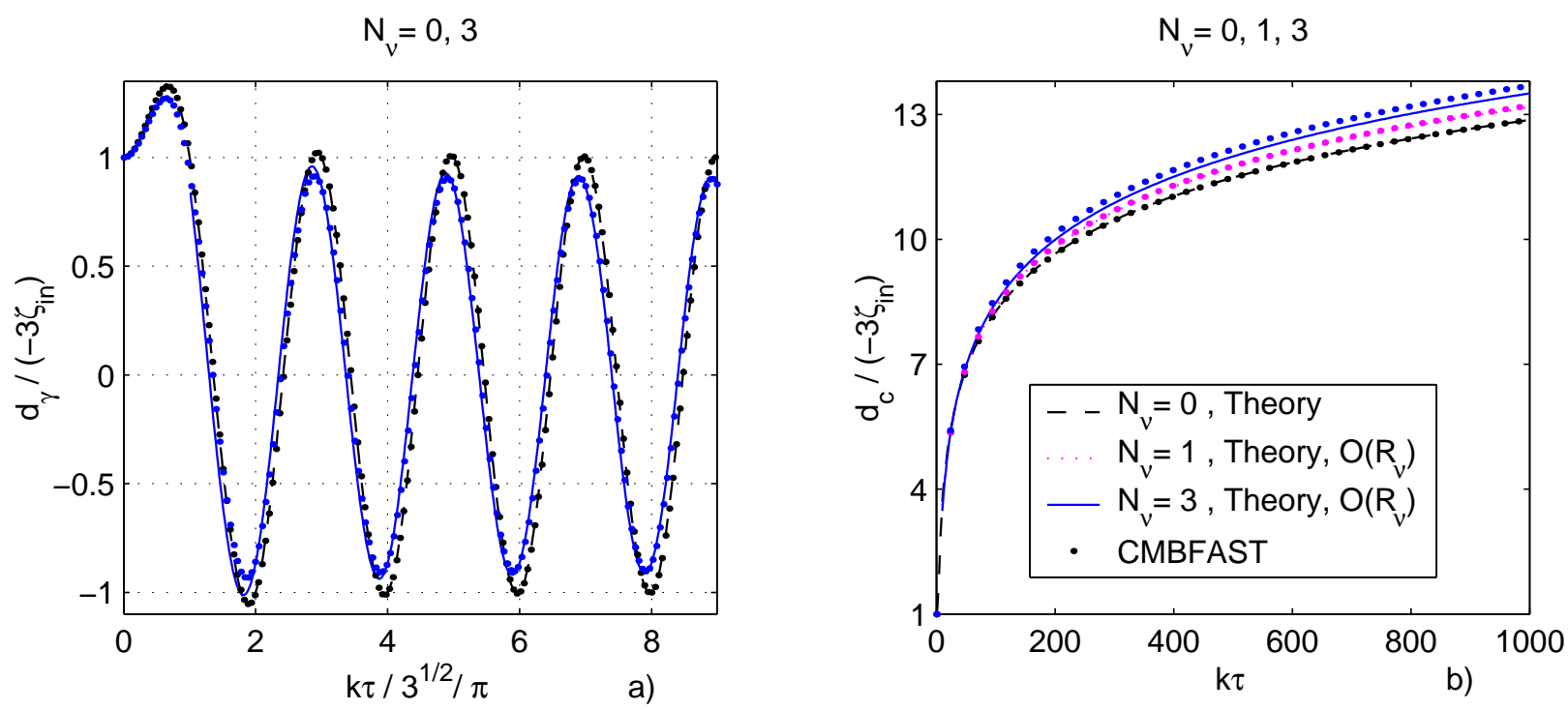

FIG. 4: a) Numerically calculated photon number density perturbation $d_{\gamma}$ in the radiation era for 0 and 3 neutrino species $N_{\nu}$ (dots) versus the theoretical prediction (117) for $N_{\nu}=0$ (dashed) and its rescaled and phase shifted asymptotic form (121, 122) for $N_{\nu}=3$ (solid). b) Similar comparison for the dark matter density perturbation $d_{c}$ and $N_{\nu}=0,1,3$. The theoretical predictions are given by eqs. (127-129). In all the cases the $O\left(R_{\nu}^{2}\right)$ terms in the analytical formulas are neglected.

It follows from eq. (7) applied to the photon-baryon fluid with

$$
\begin{aligned}
c_{s}^{2}(\tau) & =\frac{d p_{\gamma b}}{d \rho_{\gamma b}}=\frac{1}{3\left(1+R_{b}\right)}, \\
R_{b}(\tau) & \equiv \frac{3 \rho_{b}}{4 \rho_{\gamma}} \simeq 0.6 \frac{a(\tau)}{10^{-3}}\left(\frac{\omega_{b}}{0.02}\right),
\end{aligned}
$$

Ref. [45]. The Silk damping term $2 \tau_{d} \nabla^{2} \dot{d}_{\gamma}$ in eq. (130) owes its origin to both partial photon diffusion and to the lagging of baryons behind photons in imperfectly coupled photon-baryon plasma. While these effects are minor, the damping coefficient equals [81]

$$
\tau_{d}(\tau)=\frac{\tau_{c}(\tau)}{6}\left[1-\frac{14}{15\left(1+R_{b}\right)}+\frac{1}{\left(1+R_{b}\right)^{2}}\right]
$$

where

$$
\tau_{c}(\tau) \equiv{\frac{1}{a n_{e} \sigma_{\text {Thompson }}}} .
$$

The above result for $\tau_{d}$ and eq. (130) are valid while $\tau_{c} \ll \min (1 / k, \tau)$. The damping increases substantially, Ref. [58], within the CMB last scattering surface, where the imperfect fluid approximations fail.

The general solution of eq. (130) can be obtained for subhorizon Fourier modes, $k \tau \gg 1$, using WKB approximation, Refs. [45, 82]. For the monopole of the photon effective temperature perturbation

$$
\Theta_{0, \gamma}^{\mathrm{eff}} \equiv \frac{\left\langle\delta T_{\gamma}(\tau, \mathbf{r}, \hat{\mathbf{n}})\right\rangle_{\hat{\mathbf{n}}}}{T_{\gamma}}+\Psi=\frac{1}{3} d_{\gamma}+\Phi+\Psi,
$$

it gives

$$
\Theta_{0, \gamma}^{\mathrm{eff}} \simeq A \frac{e^{-k^{2} x_{S}^{2}}}{\left(1+R_{b}\right)^{1 / 4}} \cos (k S+\delta \varphi)-R_{b} \Phi
$$

where the size of the acoustic horizon $S$ and the Silk damping length $x_{S}$ equal

$$
S(\tau) \equiv \int_{0}^{\tau} c_{s} d \tau^{\prime}, \quad x_{S}^{2}(\tau) \equiv \int_{0}^{\tau} \tau_{d} d \tau^{\prime}
$$

The solution (135-136) takes into account that on the subhorizon scales photon-baryon fluid and neutrinos contribute negligibly to the gravitational potentials $\Phi$ and $\Psi$, primarily generated by CDM. Hence, these potentials do not vary substantially over a single period of acoustic oscillations. It also assumes that $\tau_{d} \ll 1 / k$, which is a necessary condition for the validity of eqs. (130) and (132).

The photon-baryon plasma velocity potential, affecting the CMB anisotropy through the Doppler effect, is easily found from eq. (4). For Fourier modes, 


$$
u_{\gamma}=-\frac{\dot{d}_{\gamma}}{k^{2}} \simeq-\frac{3 \dot{\Theta}_{0, \gamma}^{\mathrm{eff}}}{k^{2}} \simeq \frac{A \sqrt{3}}{k} \frac{e^{-k^{2} x_{S}^{2}}}{\left(1+R_{b}\right)^{3 / 4}} \sin (k S+\delta \varphi)
$$

where the last two equalities are valid within the approximations that were applied to WKB result (135).

The present CMB temperature anisotropy observed in the direction $\hat{\mathbf{n}}$,

$$
\frac{\delta T(\hat{\mathbf{n}})}{T} \equiv \Theta(\hat{\mathbf{n}}) \equiv \sum_{l=0}^{\infty} \sum_{m=-l}^{l} \Theta_{l m} Y_{l m}(\hat{\mathbf{n}})
$$

can be written as the line of sight integral [42]:

$$
\Theta(\hat{\mathbf{n}})=\int_{0}^{\tau_{0}} d \tau\left[\dot{g}\left(\Theta_{0, \gamma}^{\mathrm{eff}}-v_{\gamma}^{i} n_{i}+Q^{i j} n_{i} n_{j}\right)+g(\dot{\Phi}+\dot{\Psi})\right]_{\tau, \mathbf{r}=\left(\tau_{0}-\tau\right) \hat{\mathbf{n}}} .
$$

Here, for scalar perturbations, $\Theta_{0, \gamma}^{\text {eff }}$ is given by eq. (134), $v_{\gamma}^{i}=-\nabla^{i} u_{\gamma}$, and $Q^{i j}=\left(\nabla^{i} \nabla^{j}-\frac{1}{3} \delta^{i j} \nabla^{2}\right) q$, where $q$, negligible during the tight coupling, is a linear combination of $\pi_{\gamma}$ and multipole potentials that describe photon polarization. The perturbations $\Theta_{0, \gamma}^{\mathrm{eff}}, u_{\gamma}$, and $q$ are evaluated along the line of sight $\mathbf{r}(\tau)=\left(\tau_{0}-\tau\right) \hat{\mathbf{n}}$, assuming the observer is positioned at the origin. The "integral visibility function" $g(\tau)$ is the probability for a CMB photon to propagate unscattered from the time $\tau$ to the present time $\tau_{0}$ :

$$
g(\tau)=\exp \left[-\int_{\tau}^{\tau_{0}} \frac{d \tau^{\prime}}{\tau_{c}\left(\tau^{\prime}\right)}\right]
$$

Expanding the expression under the line of sight integral (139) over Fourier harmonics in a flat universe, we have

$$
\Theta(\hat{\mathbf{n}})=\int_{0}^{\tau_{0}} d \tau \int \frac{d^{3} \mathbf{k}}{(2 \pi)^{3}} \zeta_{\text {in }}(\mathbf{k}) T_{\Theta}(\tau, k) e^{i\left(\tau_{0}-\tau\right) \hat{\mathbf{n}} \cdot \mathbf{k}} .
$$

The transfer function $T_{\Theta}$ in the above equation is constructed from the perturbation Fourier modes, normalized by $\zeta(\tau \rightarrow 0, \mathbf{k})=1$, as

$$
T_{\Theta}(\tau, k) \equiv \dot{g}\left[\Theta_{0, \gamma}^{\mathrm{eff}}+u_{\gamma} \frac{\partial}{\partial \tau_{0}}+q\left(\frac{\partial^{2}}{\partial \tau_{0}^{2}}+\frac{1}{3} k^{2}\right)\right]+g(\dot{\Phi}+\dot{\Psi})
$$

Given that for primordial fluctuations $\left\langle\zeta_{\text {in }}(\mathbf{k}) \zeta_{\text {in }}^{*}\left(\mathbf{k}^{\prime}\right)\right\rangle=(2 \pi)^{3} \delta_{\mathrm{D}}^{(3)}\left(\mathbf{k}-\mathbf{k}^{\prime}\right) P_{\zeta}(k)$ and that a plane harmonic projects onto a spherical one as $\int d^{2} \Omega_{\hat{\mathbf{n}}} Y_{l m}^{*}(\hat{\mathbf{n}}) e^{i \hat{\mathbf{n}} \cdot \mathbf{x}}=4 \pi Y_{l m}^{*}(\hat{\mathbf{x}}) i^{l} j_{l}(x)$, the CMB temperature auto-correlation function $C_{l}^{T T} \equiv$ $\left\langle\left|\Theta_{l m}\right|^{2}\right\rangle$ becomes

$$
C_{l}^{T T}=\frac{2}{\pi} \int k^{2} d k P_{\zeta}(k)\left|\int_{0}^{\tau_{0}} d \tau T_{\Theta}(\tau, k) j_{l}\left(k\left(\tau_{0}-\tau\right)\right)\right|^{2}
$$

When CMB polarization or other cosmological anisotropies are accessible, additional two-point correlations can be considered, such as $C_{l}^{E E}$ or $C_{l}^{T E}$ for the linear polarization component $E$ generated by scalar perturbations, Ref. [83]. The observed CMB polarization can be expressed similarly to eq. (139) as a line of sight integral [50] over the perturbations that source the photon polarization. Likewise, the corresponding $C_{l}$ 's are given by a $d k$ integral of the product of the power spectrum $P_{\zeta}(k)$ and two time convolutions of perturbation variables with $j_{l}\left(k\left(\tau_{0}-\tau\right)\right)$ or its derivatives.
Any contribution to the correlation functions $C_{l}$ from the time of last scattering is characterized by $\tau \ll \tau_{0}$. The corresponding Bessel functions vanish exponentially when their argument $k\left(\tau_{0}-\tau\right) \simeq k \tau_{0}$ is less than $l$, given $l \gg 1$. Thus the related to the acoustic oscillations constituents of $C_{l}$ are essentially affected by only the modes that enter the horizon well before the radiation-matter equality if $l \gg \tau_{0} / S\left(\tau_{e}\right) \approx 230$, with $\tau_{e}$ given by eq. (49) and WMAP best fit parameters [46]. 
Neutrino signatures and degeneracies

For the modes that enter the horizon in the radiation era, both the WKB solution (135-136) and the radiation era solution (121) should be valid over a positive time interval $1 / k \ll \tau \ll \tau_{e}$. Then the comparison of eq. (135), where $R_{b}$ and $x_{S}^{2}$ are negligible in the radiation era, with formulas $(134,121)$ shows that in eq. (135):

a) The phase shift $\delta \varphi$ is given by eq. (122);

b) The integration constant $A$ equals

$$
A=\zeta_{\text {in }}\left(1+\Delta_{\gamma}\right)
$$

The Fourier modes of plasma velocity potential (137) acquire the same phase shift and same multiplicative change of the amplitude relative to the neutrinoless case.

The phase shift of the photon acoustic oscillations on subhorizon scales can not be produced from adiabatic primordial fluctuations by any dynamics involving only the photon-baryon plasma and non-relativistic species. This is seen easily in real space by noting that the acoustic oscillations correspond to small-scale, appearing singular on the Hubble scale, features in the photon Green's function. We found in Sec. IV E that in the radiationdominated universe where no perturbations can propagate faster than the acoustic speed the only such features would be the delta function spikes in eq. (116) at $x= \pm \tau / \sqrt{3}$.

The spikes would continue propagating away from the perturbation origin with the speed $c_{s}(\tau)$ of eq. (131) past the radiation era until the recombination, as, by the general equivalence principle, their local acoustic dynamics could not be altered by the gravity of perturbations of other species. By the time of recombination, the singular part of the photon density Green's function would have the form

$$
d_{\gamma, \operatorname{sing}}(\tau, x)=D(\tau) \delta_{D}(|x|-S(\tau))
$$

The amplitude $D(\tau)$ depends on the expansion rate of the photon background. The calculations of Ref. [56] in real space show that $D(\tau) \propto e^{-k^{2} x_{S}^{2}} /\left(1+R_{b}\right)^{1 / 4}$. The singularity $\delta_{D}(|x|-S)$ becomes $2 \cos (k S)$ in Fourier space, i.e., without neutrinos we would recover the oscillating part of the WKB solution (135) with $\delta \varphi=0$.

Although the observed period of $C_{l}$ oscillations depends on a number of cosmological parameters, such as $\omega_{b}=\Omega_{b} h^{2}, \omega_{m}=\Omega_{m} h^{2}$, or the total $\Omega$, the oscillation phase, ideally, can be extracted independently of the period. In practice, caution is required. Indeed, even in the neutrinoless model the location of the acoustic peaks in $C_{l}$ is not exactly proportional to the wave number $k$ of the extrema of the effective temperature transfer function (135). This is caused by a variety of effects, listed in Sec. 8.3.2 of Ref. [84]. Most of them vanish in the $l \rightarrow \infty$ limit but only as negative powers of $l$ and are still sufficiently important to modify quantitative predictions for $C_{l}$ phase even at $l \sim 3000$. Two of the effects remain finite for arbitrarily high $l$. First, the Bessel function in eq. (143), or a similar equation for polarization, is exponentially cut off for $l>k r$, where $r \equiv \tau_{0}-\tau_{\gamma \text { dec }}$ in the flat model. But $\left\langle\left|j_{l}\right|^{2}\right\rangle$ decreases slowly, by a power law, for $l<k r$. As a consequence, an extremum of the temperature perturbation transfer function (135) at a certain $k$ contributes most to the $C_{l}$ with $l$ somewhat less than $k r$. This shifts $C_{l}$ peaks toward lower l's: $l_{n^{\prime} \text { th peak }}=\left(\pi r / S\left(\tau_{\gamma \text { dec }}\right)\right)\left(n-\phi_{n}\right)$, with $\phi_{n}>0$. Fortunately, the corresponding shift $\phi_{n}$ for $C_{l}^{T T}$ approaches a model independent constant value 1/8, Ref. [84]. Second, the phase of $C_{l}$ oscillations is obscured by the rapid decrease of $C_{l}$ magnitude for $l \gtrsim 10^{3}$ due to the Silk damping and smoothing of the anisotropies by the nonzero width of the last scattering surface. Nevertheless, the numerical analysis of Sec. VI shows the robustness of the phase under the change of any standard parameter other than the neutrino density. The phase shift signature enables one to constraint $N_{\nu}$ tightly when a sufficiently large $l$ interval is accessible.

The rescaling of the photon-baryon oscillation amplitude by $1+\Delta_{\gamma}<1$ for $k \gg 1 / S\left(\tau_{e}\right)$ causes the same rescaling of all the photon phase space density and polarization multipoles, which develop during the decoupling. The non-oscillating part of the gravitational potentials, also affecting the CMB anisotropy by eq. (135) and (139), is however generated by CDM density perturbations and rescales differently, by $1+\Delta_{c}>1$, Sec. IV F. In the square of the transfer function in eq. (143) some terms oscillate in $k$ with the period $\Delta k=\pi / S$. They come from and only from the product of two oscillating perturbations and must involve the factor $\left(1+\Delta_{\gamma}\right)^{2}<1$. There are terms that oscillate with the period $\Delta k=2 \pi / S$. They are produced by the cross-product of the oscillating photon and non-oscillating CDM generated contributions and thus get multiplied by $\left(1+\Delta_{\gamma}\right)\left(1+\Delta_{c}\right)$, which by eqs. $(122,129)$ is very close to 1 . It is hard to find a similar factor for the sum of non-oscillating terms without a detailed calculation. These terms consist of both the CDM-CDM contributions, scaling as $\left(1+\Delta_{c}\right)^{2}$, and of the non-oscillating parts of $\gamma-\gamma$ terms from the squares like $(\cos \varphi)^{2}=\frac{1}{2}+\frac{1}{2} \cos 2 \varphi$, scaling as $\left(1+\Delta_{\gamma}\right)^{2}$.

Thus we expect that for $l \gg r / S\left(\tau_{e}\right) \approx 230$ the acoustic oscillations of $C_{l}$ 's are decreased by the gravity of neutrino perturbations by the factor $\left(1+\Delta_{\gamma}\right)^{2}$. But the magnitude of the $C_{l}$ oscillations depends on other physical quantities as well.

First of all, on the primordial power spectrum $P_{\zeta}(k)$. In principle, this unknown could be excluded by comparing the CMB anisotropies with the matter density perturbations. The latter scale differently under $N_{\nu}$ variation, as seen in Sec. IV F and in the next subsection. However, given the large experimental error on the matter perturbations, a better method to exclude $P_{\zeta}$ is to compare the height of the initial acoustic peaks, entering the horizon closer to the matter era and hence less affected by neutrino perturbations, to the height of the subsequent peaks. This signature is degenerate with the 
power spectral index $n_{s} \equiv d \ln P_{\zeta} /(d \ln k)+4$ and its running in $k$, especially for only a limited number of observed peaks [34].

Second, the scaling $\left(1+\Delta_{\gamma}\right)^{2}$ is directly applicable only if one compares the models in which the photon subhorizon dynamics is identical in angular and redshift coordinates $^{12}$ since the radiation era until the present. Here is the list of the dimensionless quantities that characterize the cosmological background expansion and the local photon-baryon dynamics: ${ }^{13} 1$. The ratio of the universe expansion rates at any redshift $z$ and at the present: $H(z) / H_{0}$. It shows how distances are mapped to angles: $(z, k / h) \rightarrow l$. It also affects the photon and baryon perturbation dynamics, considered in the dimensionless variables $d z=-H(z) d \tau$ and $H_{0} d x$. This ratio,

$$
H(z) / H_{0}=\left[\Omega \rho(z) / \rho(0)+(1-\Omega) / a^{2}\right]^{1 / 2},
$$

is specified by today's total density parameter $\Omega \simeq$ $\Omega_{m}+\Omega_{\mathrm{de}}, \Omega_{m}$, the redshift of the radiation-matter equality $1+z_{\text {eq }}=\rho_{m, 0} / \rho_{r, 0}$, and the dark energy "equation of state" $w_{\mathrm{de}}(z) \equiv p_{\mathrm{de}} / \rho_{\mathrm{de}}$. 2 . The ratio of baryon and photon densities, $\rho_{b} / \rho_{\gamma}$, controlling the photonbaryon plasma dynamics. 3. The ratio of the photon free-flight time $\tau_{c}(\tau)$, eq. (132), to the particle horizon size $\tau$. This ratio determines the integral visibility function $g(z)$, eq. (140), and the important Silk damping scale, eq. (136).

The present photon density $\rho_{\gamma, 0}$ is well constrained by the COBE measurement of CMB temperature $2.725 \pm$ $0.002 K$, Ref. [74]. It is expected to redshift predictably as $\rho_{\gamma}=(1+z)^{4} \rho_{\gamma, 0}$ deep into the radiation era. The total radiation density depends on $N_{\nu}$ as $\rho_{r}=\rho_{\gamma}\left(1+\alpha_{\nu}\right)$, where $\alpha_{\nu} \simeq 0.23 N_{\nu}$, eq. (44). Given this, two models with different $N_{\nu}$ will have same ratios $\rho_{b} / \rho_{\gamma}$ and $H(z) / H_{0}$, and the related $\rho_{m, 0} / \rho_{r, 0}=z_{\text {eq }}+1$, if these models have the same $\omega_{b}=\Omega_{b} h^{2}, \Omega, \Omega_{m}$, and $w_{\mathrm{de}}(z)$, but their Hubble constants scale as $h \propto \sqrt{1+\alpha_{\nu}}$.

The two top panels in Fig. 5 show the relative change $\delta C_{l} / C_{l}$ for $\mathrm{CMB}$ temperature and polarization spectra under $N_{\nu}$ variation from 2.5 to 3.5 . The solid curves correspond to preserving the parameters $\omega_{b}, \Omega=1, \Omega_{m}$, $w_{\mathrm{de}}=-1$, and $h / \sqrt{1+\alpha_{\nu}}$, as described above, as well as the primordial power spectrum and the primordial helium fraction $Y \equiv \rho_{\mathrm{He}} / \rho_{b}$. As seen from the plots, the model with a larger $N_{\nu}$ has noticeably stronger damping on small scales. Indeed, for a fixed ionization fraction $x_{e} \equiv n_{e} / n_{H} \propto n_{e} / \omega_{H}$, where $\omega_{H}=(1-Y) \omega_{b}$ specifies

12 A universe with larger neutrino density expands faster, at least, in the radiation era. The corresponding photon temperature must, therefore, decrease faster in the time coordinate.

13 We yet ignore the somewhat relevant to $\mathrm{CMB}$ ratio $\rho_{b} / \rho_{m}$. As seen in the next subsection, this ratio is very important for matter evolution. But it affects CMB anisotropies rather mildly, through the non-oscillating CDM potential term in eq. (135) or through the CMB lensing by matter structure. the primordial hydrogen density, the ratio

$$
\frac{\tau_{c}}{\tau}=\frac{1}{\tau a n_{e} \sigma_{\text {Thompson }}} \propto \frac{h}{x_{e}(1-Y) \omega_{b} \sigma_{\text {Thompson }}}
$$

increases with $N_{\nu}$ as $h \propto \sqrt{1+\alpha_{\nu}}$.

The full conformal equivalence of the photon subhorizon dynamics can be straightforwardly implemented in numerical calculations. The dotted curves on the top panels of Fig. 5 show $\delta C_{l} / C_{l}$ when the global cosmological parameters vary as before but the numerically integrated equations in both models use the same $\tau_{c} / \tau$ and the same integral visibility function $g(z)$. The remaining $C_{l}$ change for the modes $l \gg 200$, entering the horizon before the radiation-matter equality, is uniform power suppression and a constant phase shift, as predicted for the effects of neutrino perturbations. The shift $l \rightarrow l+\delta l$ in the numerical calculations is only $80 \%$ of the expected $\delta l=\Delta l_{\text {peak }} \delta \varphi / \pi \simeq 4.6$, for the period of the acoustic oscillations $\Delta l_{\text {peak }} \simeq 300$ from Ref. [85] and $\delta \varphi$ evaluated from the radiation era result eq. (122). Likewise, the power suppression is somewhat less than the radiation era prediction $\delta\left(1+\Delta_{\gamma}\right)^{2} \simeq-4.8 \%$, with $\Delta_{\gamma}$ given by the leading term in eq. (122). These discrepancies are caused by the residual effect of the large-scale relativistic correction, c.f. the first term in eq. (117), non-negligible matter density during the horizon entry, and $O\left(R_{\nu}^{2}\right)$ corrections in eq. (122).

As suggested by eq. (147), the ratio $\tau_{c} / \tau$ in the compared models could be matched by varying the helium abundance $Y$. Apparently, when all the helium has recombined but the hydrogen remains fully ionized $\left(x_{e}=\right.$ 1 ), the quantity (147) remains constant if $Y$ varies as $(1-Y) \propto h \propto \sqrt{1+\alpha_{\nu}}$. It is less obvious that the after such a rescaling, $\tau_{c} / \tau$ coincides in both models during the recombination, when $x_{e}$ violently changes with time. Despite the complicated nature of recombination, the physical mechanism that is primarily responsible for $x_{e}$ evolution around the peak of the photon visibility function $\dot{g}$ does lead to degenerate $x_{e}(z)$ and so degenerate $\tau_{c} / \tau$.

At the redshifts $1400 \gtrsim z \gtrsim 800$, which are of the most interest, the dominating process leading to $H$ recombination to the ground state $1 s$ is the suppressed $2 s \rightarrow 1 s+\gamma+\gamma$ decay. Faster transitions to $1 s$ with the emission of a single photon do not create more hydrogen in the ground state because the emitted resonance photon soon ionizes or excites another $H$ atom in the ground state. Using the approximations of Ref. [86] (see Ref. [87] for a recent review), one can find the rate of $x_{e}$ change by assuming that due to the overcooling of the universe from the delay in recombination the fraction of all the excited $H$ atoms is negligible comparatively to the concentration of $H$ in the ground state $x_{1 s} \equiv n_{1 s} / n_{H}$ and to $x_{e}$. Then $x_{1 s}+x_{e} \simeq 1$, hence $d x_{1 s} \simeq-d x_{e}$. The change in $x_{1 s}$ is mainly due to the spontaneous decay $2 s \rightarrow 1 s+\gamma+\gamma$ with the lifetime $\Lambda \simeq 8.2 s^{-1}: d x_{1 s} / d t \simeq \Lambda x_{2 s}$. (The photo-excitation $1 s \rightarrow 2 s$ is negligible when $z<1300$.) $x_{e}$ decreases in ep recombination and increases in hydrogen photo-ionization. With realistic approximations, 

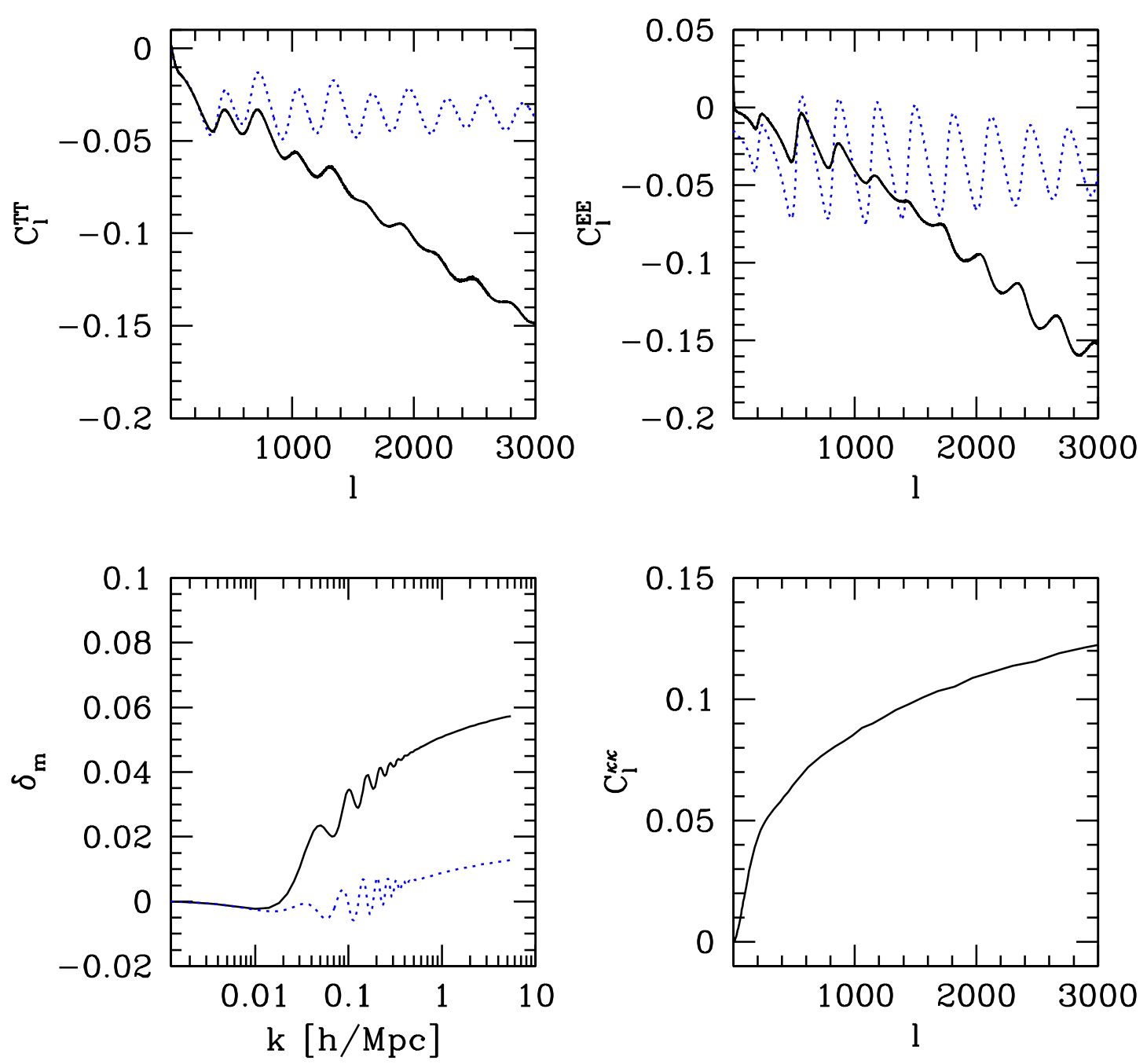

FIG. 5: The relative change in $C_{l}^{T T}$ (top left), $C_{l}^{E E}$ (top right), matter density perturbation $\delta_{m}(k / h)$ (bottom left), and $C_{l}^{\kappa \kappa}$ (bottom right) when $N_{\nu}$ varies from 2.5 to 3.5. The solid curves show the changes when all the other parameters, listed in Sec. VI, are fixed. The top two panels also show the change for fixed recombination history and equivalent Silk damping (dotted, blue). The dotted, blue curve on the bottom left panel gives the change in $\delta_{m}$ when $\omega_{b} / \omega_{m}$ is held fixed.

Ref. [86], one finds: $d x_{e} / d t=-\alpha x_{e} x_{p} n_{H}+\beta x_{2 s}$, where $x_{p} \simeq x_{e}$, and $\alpha$ and $\beta$ at a given CMB temperature, determined by the redshift, are independent of cosmological parameters. The elimination of $x_{2 s}$ from the equality of $d x_{1 s} / d t$ and $-d x_{e} / d t$ yields:

$$
\frac{d x_{e}}{d t} \simeq-\frac{\alpha \Lambda}{\beta+\Lambda} x_{e} x_{p} n_{H}
$$

When both $d / d t=-(1+z) H(z) d / d z \propto h$ and $n_{H} \propto(1-$ $Y) \omega_{b}$ vary in proportion to $\sqrt{1+\alpha_{\nu}}$, as discussed above, eq. (148) predicts that the function $x_{e}(z)$ is unchanged, and so is unchanged the last ratio in eq. (147).

Numerical calculations show that this conclusion holds very well after the end of helium recombination at $z \simeq$
1500. For a variation of $N_{\nu}$ from 2.5 to 3.5 and the corresponding adjustment of $Y$ by ${ }^{14}-0.051$, the ionization fraction $x_{e}$ changes at most by $0.5 \%$, and even twice as little within the peak of the photon visibility function $\dot{g}$. (For comparison, without the adjustment of helium abundance, the change of $x_{e}$ reaches $6 \%$ within the visibility peak.) At $2000 \lesssim z \lesssim 5000$, when helium is singly ionized, the decrease of the helium density decreases the total number of free electrons by about $2 \%$, and more

\footnotetext{
14 Astrophysical constraints on the primordial helium abundance vary among groups and span a range $Y=0.238 \pm 0.010(2 \sigma)$, Refs. [6-10], a review in Ref. [88]. Taking this prior, $Y$ needs to be considered as a free parameter in a CMB analysis only for the accuracies $\left|\Delta N_{\nu}\right| \lesssim 0.4$
} 
at higher redshifts, when the second helium electron unbounds. But the related increase of the Silk damping scale, eqs. $(132,136)$, is insignificant, and the effect is quite negligible for $d g / d z$. The change $\delta C_{l} / C_{l}$ for $T T$ and $E E$ spectra under the considered variation is seen from Fig. 6 (solid line). It is compared with the theoretical reconstruction (dashed line) obtained by shifting $C_{l}$ by $\delta l=0.8 \Delta l_{\text {peak }} \delta \varphi / \pi \simeq 3.7$ and rescaling it by $\delta\left(1+\Delta_{\gamma}\right)^{2}=-4.8 \%$. The plots show that the $Y$ adjustment removes most of the damping seen in Fig. 5.

The approximate conformal degeneracy of CMB dynamics among the models with changed dynamical time and length scales should be distinguished from the well known degeneracy of primary CMB anisotropies under any variation of $H(z)$ for $z<z_{\gamma \text { dec }}$ that preserves the angular diameter distance to the last scattering surface, Ref. [59]. The latter degeneracy is generally violated by the late ISW effect. The found one is respected by ISW but is somewhat violated by the gravity of matter perturbations, c.f. footnote 13 on p. 22, and, of course, by the gravity of neutrino perturbations.

The internal CMB dynamics is shown to be conformally invariant in an increment of $N_{\nu}$ if the helium abundance $Y$ is decreased as $\Delta Y / \Delta N_{\nu} \simeq-0.05$. In contrast, the standard Big Bang nucleosynthesis (BBN) then predicts increased ${ }^{4} \mathrm{He}$ production. ${ }^{15}$ Indeed, for $\Delta N_{\nu}>0$ the universe expands faster and less neutrons decay by the time it cools sufficiently to allow their conversion into helium. Of course, one should not presume a priori that the ratio of neutrino and photon energy densities is preserved since the nucleosynthesis until the matter era, i.e. that $N_{\nu}$ in BBN and CMB physics are equal. But if they are not, the non-degeneracy of $\mathrm{CMB}$ dynamics along the BBN-predicted curve $Y\left(N_{\nu}\right)$ makes it easier to spot the discrepancy.

Neutrino perturbations can affect the gravitational potentials even after the photon decoupling if radiation is still a significant component of the total energy density. When the gravitational potentials are time dependent, there is a contribution to the CMB anisotropy (139) that depends on the line of sight integral of $\dot{\Phi}+\dot{\Psi}$, the socalled integrated Sachs-Wolfe (ISW) effect. However, as discussed in Sec. III C, for superhorizon modes the derivative $\partial(\Phi+\Psi) / \partial R_{\nu}$ changes insignificantly after recombination in the standard models with matter radiation equality at $z \sim 3000$. Thus the modification of the ISW effect by neutrino anisotropic stress does not play a major role as a neutrino signature in CMB. Specifically, on

15 The sensitivity of the BBN yield of ${ }^{4} \mathrm{He}$ and $\mathrm{D} / \mathrm{H}$ to variation of $N_{\nu}$ and $\eta \equiv n_{B} / n_{\gamma}$ about $N_{\nu}=3$ and the WMAP [13] "best fit" value $\eta \approx 6.1 \times 10^{-10}$ is roughly

$$
\left(\begin{array}{c}
\Delta Y \\
\Delta \ln (D / H)
\end{array}\right) \approx\left(\begin{array}{cc}
0.013 & 0.01 \\
0.14 & -1.6
\end{array}\right)\left(\begin{array}{c}
\Delta N_{\nu} \\
\Delta \ln \eta
\end{array}\right)
$$

(based on the numerical results from Refs. [11, 89, 90].) large scales $\left(l<l_{1 \text { st peak }} / 3 \simeq 70\right)$ the ISW effect becomes insensitive to perturbations of relativistic neutrinos.

\section{B. Matter power spectrum}

The growth of the acoustic perturbations in the tightly coupled photon-baryon fluid is halted by photon pressure, described in eq. (7) by the term $-c_{s}^{2} \nabla^{2} d_{\gamma}$, where $c_{s} \gtrsim 1 / 2$ before recombination. Likewise, relativistic neutrinos and CMB photons after their decoupling are stabilized against gravitational collapse by the effective pressure, described by the same term with $c_{a}=1 / \sqrt{3}$, due to the velocity dispersion. In addition, as noted in the introduction, the perturbation modes of free-streaming particles on subhorizon scales decay by "directional" damping. Assuming the dark energy too does not strongly cluster on small scales, only CDM and after recombination baryons can cluster sufficiently to generate non-negligible gravitational potential inside the subhorizon. On subhorizon scales eq. (23) simplifies to $\nabla^{2} \Psi=\gamma d$. Then eqs. $(7,24)$ applied to non-relativistic species $n$ with negligible pressure $\left(c_{n}^{2} \simeq 0\right)$ give

$$
\ddot{d}_{n}+\mathcal{H} \dot{d}_{n}=\sum_{m: c_{m}^{2} \simeq 0} \gamma_{m} d_{m} \quad \text { (subhor) } .
$$

One can distinguish the following stages of the linear matter perturbation growth from their entry of the horizon in the radiation era to the present:

1. Growth of CDM perturbations in the radiationmatter universe until the recombination while baryons are tightly coupled to CMB.

2. Decoupling of the baryons from $\mathrm{CMB}$ and joining the matter gravitational collapse at $z_{d} \sim 1090$.

3. Growth of the pressureless CDM-baryon matter perturbations through the subsequent universe evolution, affected by dark energy and, possibly, global curvature.

Eq. (128) for CDM density perturbations in the radiation era on subhorizon scales can be presented as

$$
\begin{aligned}
& d_{c}^{(r, \lambda \ll \tau)}(\tau, k)= \\
& \quad=-6 \zeta_{\text {in }}\left(1+\Delta_{c}\right)\left\{A+\ln \left[(1+\delta c) \frac{k}{h}\right]\right\},
\end{aligned}
$$

where $A$ is the same function of $\tau h$ for all $k$ and $R_{\nu}$ :

$$
A(\tau h)=\ln \left(\frac{\tau h}{\sqrt{3}}\right)+\gamma-\frac{1}{2} \quad(\text { rad. era }) .
$$

At later times the matter density perturbation must be of the form

$$
d_{m}(\tau, k)=-6 \zeta_{\text {in }}\left(1+\Delta_{c}\right)\left\{A+B \ln \left[(1+\delta c) \frac{k}{h}\right]\right\},(15
$$

where $A$ and $B$ depend on $\tau h$ and on the cosmological parameters affecting the background evolution at 

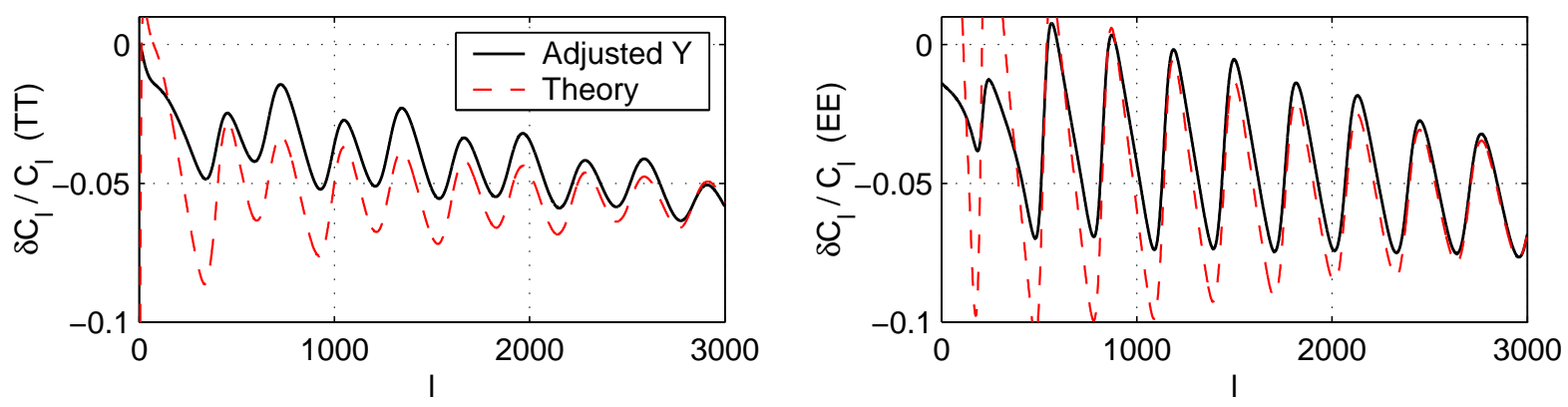

FIG. 6: The relative change in $C_{l}^{T T}$ (left) and $C_{l}^{E E}$ (right) for $N_{\nu}$ variation from 2.5 to 3.5 and the adjustment of the helium abundance $Y$ by -0.051 (solid). It is compared with the change from rescaling $C_{l}$ 's by $-4.8 \%$ and shifting them to smaller $l$ 's by $\delta l=3.7$ (dashed).

low redshift. We argue that throughout linear evolution $A$ and $B$ are independent of the mode wave-number $k$ and of $R_{\nu}$, provided that the compared models agree in $\Omega_{m}$, in $\Omega_{b}$, and in the background effective equation of state $w(z) \equiv p / \rho$. This would immediately follow from the linearity of the evolution with the initial conditions (150) in the radiation era if the equations of matter dynamics in terms of the dimensionless variable $\tau H_{0} \propto \tau h$ coincide. The latter is manifestly so by eqs. $(149,42)$ for the stages 1 and 3 of the three stages of linear matter clustering identified above. As for the stage 2 , the scale and $R_{\nu}$ invariances of the dynamics are violated by the small oscillations of the baryon density and velocity at the decoupling due to the acoustic oscillations in CMB, Ref. [2]. However, this effect becomes negligible at sufficiently small scales because of the Silk damping. Hence we assume that the baryons decouple with initially negligible density and velocity perturbations. Then all the stages of the matter evolution are scale- and $R_{\nu}$-invariant. Then, from eq. (152), the matter density perturbation modes $d_{m}\left(z, k, R_{\nu}\right)$ can be obtained from the modes in the model with $R_{\nu} \rightarrow 0^{16}$ and the identical $\Omega_{m}, \Omega_{b}$, and $w(z)$ as

$$
d_{m}\left(z, \frac{k}{h}, R_{\nu}\right)=\left(1+\Delta_{c}\right) d_{m}^{\left(R_{\nu} \rightarrow 0\right)}\left(z,(1+\delta c) \frac{k}{h}\right)
$$

where for the considered small scales, entering the horizon in the radiation era, $\Delta_{c}$ and $\delta c$ are given by eq. (129).

This effect of neutrino perturbations on the matter density (dotted curve on the left bottom panel of Fig. 5) is too small to provide by its own a useful information about $N_{\nu}$ from the available cosmological probes.

16 In the models with dark matter, cosmological constant, global curvature, but negligible baryon density $\left(\rho_{b} / \rho_{c} \ll 1\right)$, the linear matter density perturbation well after the radiation era on the small scales is

$d_{m}^{\left(R_{\nu} \rightarrow 0\right)}=d_{\text {in }}\left[\ln \left(\frac{4 k \tau_{e}}{\sqrt{3}}\right)+\gamma-\frac{7}{2}\right] \frac{15 H(a)}{2 a_{\mathrm{eq}} H_{0}} \int_{0}^{a} d a^{\prime}\left(a^{\prime} \frac{H\left(a^{\prime}\right)}{H_{0}}\right)^{-3}$,

where $d_{\text {in }}=-3 \zeta_{\text {in }}$. Analytical, albeit more complicated, expressions also exist for non-negligible baryon density [2, 82].
A realistic data analysis constraining the abundance of ultra-relativistic neutrinos should also include CMB data. However, the variations of CMB spectra with $N_{\nu}$, Sec. VA, are less contaminated by neutrino unrelated physics if $\omega_{b}=\Omega_{b} h^{2}$, rather than $\Omega_{b}$, is fixed. Likewise, the Big Bang Nucleosynthesis (BBN) constraints $\omega_{b}$ and not $\Omega_{b}$. Thus it is more practical to consider the variation of matter density modes with $N_{\nu}$ in the direction of the maximal $\mathrm{CMB}$ and $\mathrm{BBN}$ degeneracies - under a fixed $\omega_{b}$.

The magnitude of matter perturbations after the radiation era is sensitive to the parameter

$$
\beta \equiv \frac{\rho_{b}}{\rho_{c}+\rho_{b}}=\frac{\omega_{b}}{\omega_{m}}
$$

e.g. Ref. [2], which is currently estimated as $0.17 \pm$ 0.01 [13]. One reason for this sensitivity is the slower growth of CDM perturbations prior to the recombination in the model in which a larger fraction $\beta$ of the nonrelativistic matter is withheld from gravitational collapse by the photon pressure. The other reason is a greater dilution of the growing CDM perturbations by almost unperturbed baryons when the latter finally decouple from photons.

It is apparently impossible to change the neutrino density preserving all of the parameters $\omega_{m} / \omega_{r}, \omega_{b}$, and $\beta$. If one varies $R_{\nu}$ while keeping $1+z_{\mathrm{eq}}=\omega_{m} / \omega_{r}$ and $\omega_{b}$ fixed, to minimize the changes in CMB spectra, then the parameter $\beta$ will vary. For example, $\beta$ decreases by approximately $41 \%$ for $N_{\nu}$ change from 0 to 3 , and by $14 \%$ for $N_{\nu}$ variation by 1 around its standard value 3.04 . This is yet another source of breaking the degeneracies between $N_{\nu}$ and the other cosmological parameters. The significance of $\beta$ variation for the growth of matter perturbations is evident from comparing the dotted (fixed $\Omega_{b}$ and $\Omega_{m}$ ) and solid (fixed $\omega_{b}$ and $\Omega_{m}$ ) curves in Fig. 5 .

\section{FORECASTS FOR FUTURE EXPERIMENTS}

In this section we use numerical solutions from CMBFAST and apply them to predict the precision to which 
$N_{\nu}$ can be constrained from future experiments. We follow the basic approach of Ref. [91]: we evaluate the standard error in a cosmological parameter $s_{i}$ as $\Delta s_{i}=$ $\left(\boldsymbol{\alpha}^{-1}\right)_{i i}^{1 / 2}$, where $\boldsymbol{\alpha}$ is the Fisher matrix:

$$
\alpha_{i j}=\sum_{l} \sum_{X, Y} \frac{\partial C_{l}^{X}}{\partial s_{i}} \operatorname{Cov}^{-1}\left(\hat{C}_{l}^{X}, \hat{C}_{l}^{Y}\right) \frac{\partial C_{l}^{Y}}{\partial s_{j}} .
$$

Here, $\mathrm{Cov}^{-1}$ is the inverse of the covariance matrix, $s_{i}$ are the unconstrained cosmological parameters, and $X, Y$ stand for the observable power spectra. We limit the analysis to CMB spectra $T T, E E, T E$, and lensing convergence power spectrum $\kappa \kappa$ as measured from CMB itself. For each $l$, one has to invert the covariance matrix and sum over $X$ and $Y$. The derivatives were calculated by finite differences. The step was usually taken to be about $5 \%$ of the value of each parameter and symmetric around the pivot point at the best fitted WMAP model, Ref. [13].

An experiment is fully characterized by its sky coverage $f_{\text {sky }}$, temperature, polarization, and convergence instrument (or reconstruction) noise $w_{T}^{-1}, w_{P}^{-1}$, and $w_{\kappa}^{-1}$ and by the beam smearing window function $B_{l}^{-2}=$ $e^{l(l+1) \theta_{b}^{2} /(8 \ln 2)}$, with $\theta_{b}$ measuring the width of the beam. For example, the covariance element for $T T$ is given by

$\operatorname{Cov}\left(\hat{C}_{l}^{T T}, \hat{C}_{l}^{T T}\right)=\frac{2}{(2 l+1) f_{\mathrm{sky}}}\left(C_{l}^{T T}+w_{T}^{-1} B_{l}^{-2}\right)^{2}$.

The full covariance matrix for the CMB power spectra is given in Ref. [92]. For the lensing convergence noise spectrum we take the values from the maximum likelihood method developed in Ref. [93], which gives the lowest reconstruction noise. In addition, we impose a maximum $l_{\max }=3000$ cutoff on all the spectra and we do not include the information from higher $l$. The justification for this is that scattering off moving electrons during and after reionization leads to an additional component in the CMB that cannot be separated from the $\mathrm{CMB}$ on the basis of frequency information. This component is rather uncertain since it receives contributions from perturbative and nonlinear structures, as well as from the patchiness in ionizing fraction. It is expected to be significantly less important for polarization, so our approach may be conservative for polarization sensitive experiments.

In our analysis we use parameters for several future experiments. While WMAP constraints on $N_{\nu}$ are not yet competitive with the nucleosynthesis limits, Planck satellite will improve the sensitivity of WMAP by an order of magnitude. In parallel, there will be ground based experiments, such as $\mathrm{SPT}^{17}$ or the proposed $\mathrm{ACT}^{18}$ that will extend Planck to smaller angular scales. Whether or not

17 http://astro.uchicago.edu/spt/

18 http://www.hep.upenn.edu/ angelica/act/act.html they will be polarization sensitive remains to be determined, so we explore both possibilities. Finally, we also explore the prospects of an ambitious high resolution, low noise satellite dedicated to polarization (CMBPOL), which will be able to measure with high accuracy not only CMB temperature and polarization but also matter power spectrum using the weak lensing induced signal in higher order correlations. We also explore if adding information from more local probes, such as weak lensing, can further reduce the uncertainties.

We explore 11 parameters in our analysis. These are $\left\{\omega_{m} / \omega_{r}, \omega_{b}, \omega_{\nu \text { massive }}, N_{\nu}, \Omega_{\mathrm{de}}, w_{\mathrm{de}}, \tau_{\text {reion }}, P, n_{s}, n_{s}^{\prime}, Y\right\}$. The first one is proportional to the ratio of matter density to radiation density (photons plus neutrinos), the second to proper baryon density, and the third to the proper density of massive neutrinos. $\Omega_{\mathrm{de}}$ is the dark energy density relative to the critical density, $w_{\text {de }}$ is its effective constant equation of state $p_{\text {de }} / \rho_{\text {de }}, \tau_{\text {reion }}$ is the reionization optical depth, $P$ is the amplitude of curvature power spectrum at $k=0.05 / \mathrm{Mpc}$, and $n_{s}$ and $n_{s}^{\prime}=d n_{s} / d \ln k$ are the scalar spectral index and its running at $k=0.05 / \mathrm{Mpc}$. We do the analysis for both fixed and unconstrained helium abundance $Y$.

We ignore tensor perturbations since their contribution is limited to large scales, where neutrinos do not play a major role. We also ignore $B B$ power spectrum, which is useful primarily as a tracer of tensors. (On small scales it can be a useful tracer of matter power spectrum and can provide additional constraints on the convergence power spectrum as extracted from the nongaussianities in CMB; we ignore this additional information here.) We use the basis functions $\partial C_{l}^{X} / \partial s_{i}$ as defined by these parameters, but we also explore any possible numerical instabilities due to the variation in the angular scale of the acoustic horizon, which is affected by variations in several of the parameters.

Fig. 5 shows the $N_{\nu}$ derivatives of $C_{l}^{X}$ for $X=T T$, $E E, \kappa \kappa$, and of the matter fluctuation $\delta_{m}$. The derivatives of CMB spectra with lensing are plotted, but the unlensed ones are qualitatively similar and the wiggles are not due to lensing, but due to the phase shift (the lensed derivatives were used in the Fisher matrix analysis). The solid curves on the $C_{l}$ plots show the $N_{\nu}$ derivatives with the other parameters kept fixed. As discussed in previous sections, changing the effective number of neutrinos changes not only the initial phase and amplitude of the acoustic oscillations but also the angular scales of the acoustic horizon and oscillation damping. To separate these effects, we also show with the dotted, blue line the change in the spectra while keeping the visibility function and the photon free flight length in the units of $H_{0}^{-1}$ unchanged at every redshift.

The modification of CMB spectra due to the change in the angular scale of acoustic oscillations is described by multiplicative rescaling $l \rightarrow\left(1-0.002 \Delta N_{\nu}\right) l$. By itself, it is degenerate with other effects that change the angular acoustic scale, such as variation of $w_{\mathrm{de}}$. The combined effect of the additive and multiplicative phase shifts is such 

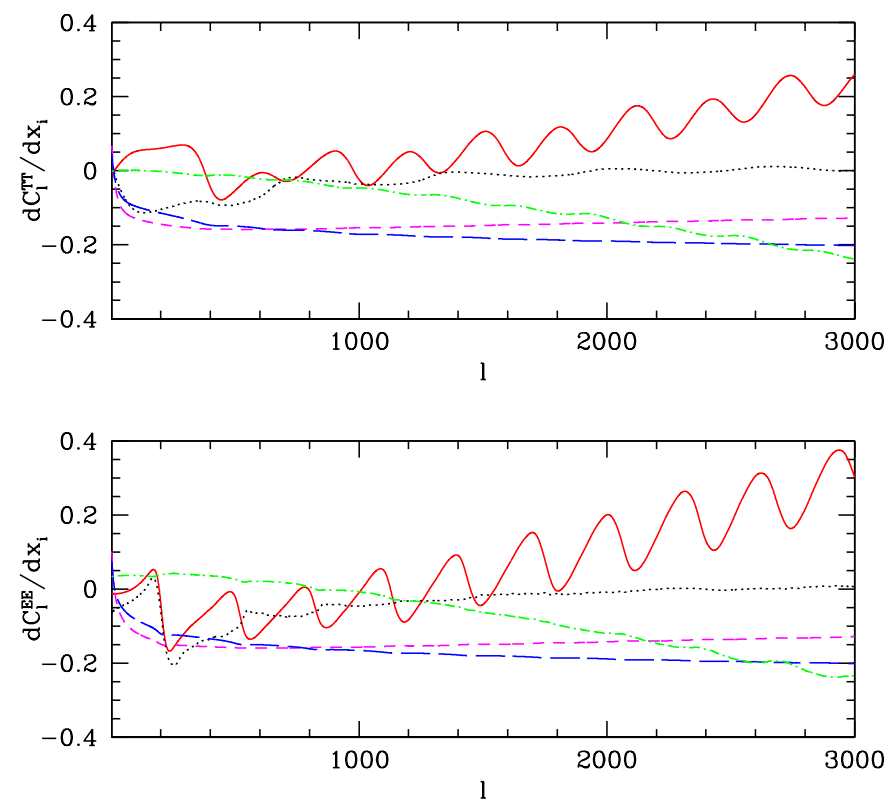

FIG. 7: The derivatives of $C_{l}^{T T}$ (top) and $C_{l}^{E E}$ (bottom) with respect to $\omega_{b}$ (solid, red), $\omega_{m} / \omega_{r}$ (dotted, black), $d n_{s} / d \ln k$ (short-dashed, cyan) and $n_{s}$ (long dashed, blue) and $Y$ (dotted-dashed, green). All were shifted to match the angular scale of acoustic horizon. This explains lack of oscillations when varying $\omega_{m} / \omega_{r}$ or $Y$. Varying $\omega_{b}$ changes the amplitude of the oscillations, so adjusting the angular scale does not make their change vanish. Note that the variation in $Y$ appears as a change in damping length.

that the phase shifts cancel exactly at $l \sim 1500$, but not at other values of $l$. For the temperature anisotropies, the phase shift is barely visible since temperature oscillations are weaker due to the competing effects from density and velocity terms. They are further suppressed by lensing. For polarization, which has more prominent acoustic oscillations, the phase shift remains visible and can be clearly distinguished from the change in angular size of the acoustic horizon. This suggests that polarization information is crucial in extracting neutrino signature. Quantitative analysis in Table III confirms that. Note that $\partial C_{l} / \partial N_{\nu}$ approach zero at low $l$. There is no significant neutrino dependent contribution coming from the integrated Sachs-Wolfe term at the low $l$, as discussed in Secs. III C and V A.

In Fig. 7 we show the derivatives of $C_{l}^{T T}$ and $C_{l}^{E E}$ with respect to some of the other parameters. The displayed derivative are for $\omega_{m} / \omega_{r}, \omega_{b}, n_{s}, d n_{s} / d \ln k$, and $Y$ and they are taken by keeping the angular scale of the acoustic horizon constant. Other derivatives are trivial and we do not show them here: the amplitude and reionization optical depth only rescale the amplitude, while dark energy density and its equation of state only change the angular scale of the acoustic horizon and make no effect after this change is taken out. Massive neutrinos have a minor effect on $\mathrm{CMB}$ over the range of interest.
It is evident that none of these can mimic the additive phase shift generated by neutrinos. This signature thus uniquely identifies the presence of neutrinos in the context of adiabatic initial conditions.

If matter power spectrum information is available, one can exploit the fact that while neutrino perturbations suppress $\mathrm{CMB}$ anisotropies, they enhance the matter power spectrum, Figs. 4 and 5. For $\Delta N_{\nu}=1$ at a fixed $\omega_{b}, k / h=1 \mathrm{Mpc}^{-1}$, and $z=0$ this gives $\Delta P_{m} / P_{m} \approx$ 0.12 , of which 0.02 is due to neutrinos alone and the rest to the variation of $\omega_{b} / \omega_{m}$, as discussed in Sec. VB. At the same time, the CMB power spectra are suppressed by $15 \%$ at $l=3000$ if $Y$ is fixed. Whether or not this is a useful method to break the degeneracies depends on the accuracy with which matter power spectrum can be extracted. We performed the analysis for the experiments in Table III assuming weak lensing information as can be extracted from the CMB itself. A Fisher matrix analysis with lensing reconstructed convergence power spectrum using minimum variance maximum likelihood errors [93] fails to improve significantly the accuracy on $N_{\nu}$, even with the polarization information, which allows for a better reconstruction of the convergence spectrum. This is in contrast to other parameters such as massive neutrinos, for which lensing information significantly improves the bounds [94].

Unless the astrophysical constraints on the primordial helium abundance $Y$ will improve by an order of magnitude, $Y$ should be included in the list of unknown cosmological parameters for the accuracies of the order of $\Delta N_{\nu} \sim 10^{-1}$. On the other hand, if we assume that the neutrino fraction of the radiation is unchanged between $\mathrm{BBN}$ and recombination and there are no significant $\nu / \bar{\nu}$ asymmetries then BBN tightly limits the helium fraction, to $\Delta Y \sim 10^{-3}$ or better $[11,12]$. For this reason, we performed the parameter prognosis with and without $Y$ parameter. As found in Sec. V A, an adjustment of $Y$ can compensate the changes in the acoustic and damping scales due to neutrino density but it preserves the phase shift signature due to the gravity of neutrino perturbations. Correspondingly, the CMB limits on $N_{\nu}$ somewhat broaden but remain tight even with the unknown value of $Y$. Interestingly, the CMB data itself can be used to constraint the primordial helium abundance independently of the astrophysical measurements and the related systematic uncertainties. For the considered CMBPOL experiment with the polarization data included we find $\sigma(Y) \simeq 0.005$ from the CMB alone.

\section{CONCLUSIONS}

In this paper we study analytically the evolution of cosmological perturbations in the presence of ultrarelativistic neutrinos. While dynamical equations for cosmological perturbations have been known for a while [3, 57, 95-97], their analytical solutions exist only in a handful of cases and are restricted to the fluid description. 
ERror ForecASTS

\begin{tabular}{|c|c|c|c|c|c|c|c|}
\hline Experiment & $f_{\text {sky }}$ & $\theta_{b}$ & $\begin{array}{c}w_{T}^{-1 / 2} \\
{\left[\mu \mathrm{K}^{\prime}\right]}\end{array}$ & $\begin{array}{c}w_{P}^{-1 / 2} \\
{\left[\mu \mathrm{K}^{\prime}\right]}\end{array}$ & $\begin{array}{c}\Delta N_{\nu} \\
\mathrm{TT}\end{array}$ & $\begin{array}{c}\Delta N_{\nu} \\
\mathrm{TT}+\mathrm{TE}+\mathrm{EE}\end{array}$ & $\begin{array}{c}\Delta N_{\nu}(\text { free } Y) \\
\mathrm{TT}+\mathrm{TE}+\mathrm{EE}\end{array}$ \\
\hline Planck & 0.8 & $7^{\prime}$ & 40 & 56 & 0.6 & 0.20 & 0.24 \\
ACT & 0.01 & $1.7^{\prime}$ & 3 & 4 & 1 & 0.47 & 0.9 \\
ACT + Planck & & & & & 0.4 & 0.18 & 0.24 \\
CMBPOL & 0.8 & $4^{\prime}$ & 1 & 1.4 & 0.12 & 0.05 & 0.09 \\
\hline
\end{tabular}

TABLE III: Standard deviations on $N_{\nu}$ as expected from Planck, ACT, Planck+ACT and CMBPOL using temperature data only $(T T)$ and added polarization $(T T+T E+E E)$. The primordial helium abundance $Y$ is considered a priori unconstrained for the last column results and fixed by independent measurements in the preceding two columns. Adding weak lensing convergence as reconstructed from CMB $(T T+T E+E E+\kappa \kappa)$ does not significantly improve the bounds, even assuming polarization information is available.

The best known examples, e.g. Ref. [60], are the solutions for CDM and photon-baryon plasma in the matter and radiation eras or in the subhorizon limit, and for superhorizon metric perturbations. In contrast, neutrinos cannot be modeled by a fluid and their phase space distribution should be considered.

Most of the recent publications abandoned the analytic approaches and relied on numerical results from Boltzmann integrator codes. While, in principle, there is nothing wrong with this, analytic solutions often lead to deeper understanding of the problem that can reveal the new directions of exploration. They sharpen the focus on the features that are unique and cannot be mimicked by the variation of other parameters. Care must be exercised when performing numerical analysis and parameter forecasting for future experiments. The computational errors must be well controlled, otherwise they can lead to artificial breaking of degeneracies. Besides, the parameter space of forecasting is often small and with the addition of new parameters new degeneracies may open up. For example, while so far only simple parameterization of the primordial power spectrum have been explored, one could consider its more general parameterization, including the running of the slope, its running, etc. as free parameters. In this case analytic solutions can provide a better understanding of whether the limits on a given parameter are robust against adding new parameters.

We obtain analytic perturbative in $\rho_{\nu} / \rho_{r}$ solutions for cosmological perturbations in the presence of ultrarelativistic neutrinos. Much of the success is due the following result, equally useful for fluid models. We find that a simple redefinition of the independent dynamical variables that is consistent with their classical interpretation and preserves them on small scales, eliminates all the time derivatives of the non-dynamical metric perturbations from the evolution equations in the Newtonian gauge. The resulting description of cosmological perturbations acquires the advantages of an initial value Cauchy's problem while remains formulated in the Newtonian gauge, which is fully fixed and is especially suitable for describing the physics of CMB. Moreover, it turns out that even in the solvable fluid models the solutions for matter or radiation density perturbations, c.f. eqs. $(117,127)$, appear far simpler in the redefined variables. In addition, these variables are generally constant on superhorizon scales.

While most of the previous literature has focused on Fourier space analysis, we also consider perturbation Green's functions in real space $[55,56]$. The latter become indispensable for the analytical study of neutrino perturbations. They also allow one to prove quickly that without neutrinos the cosine form of the acoustic density oscillations in the radiation era cannot be modified by the gravitational feedback processes.

We use the zero, in the powers of $\rho_{\nu} / \rho_{r}$, order solutions for neutrino perturbations to derive the analytic expressions for the $\mathrm{CMB}$ and matter density fluctuations in the linear order. We show that these first order solutions are for the most part sufficient for a quantitative interpretation of numerical solutions. Finally, we use the full numerical solutions from CMBFAST to derive parameter forecasts for various planned experiments. The presented methods can be straightforwardly extended to other applications such as tensor modes and massive neutrinos. We plan to address some of these in future work.

The distinctive cosmological features of ultrarelativistic neutrinos are due to their free streaming at the speed of light. The free streaming creates neutrino anisotropic stress, perturbing Newtonian metric even for superhorizon modes. In real space, it also leads to perturbing the photon-baryon plasma beyond the acoustic horizon of a primordial perturbation. In Fourier space this manifests itself as the phase shift in the acoustic oscillations that is generated at horizon crossing. This phase shift is unique in the sense that for adiabatic perturbations no non-relativistic or fluid matter can generate it. The effect changes the phase additively and corresponds to $\Delta l \sim-4$ for $\Delta N_{\nu}=1$. In contrast, any change in the angular scale of acoustic horizon acts as multiplicative rescaling $l \rightarrow \alpha l$. The two shifts are only degenerate at a single $l$ and can be distinguished in general, Fig. 5. The effect is more visible in polarization, which has sharper acoustic peaks relative to temperature anisotropy, where the density and velocity contributions to the $C_{l}$ oscillations partially cancel. As a result, the precision of determining the effective number of neutrino 
species can be improved dramatically if polarization information is included.

Phase shift is not the only signature of neutrinos in CMB. The free streaming neutrinos also suppress the oscillation amplitude of the CMB modes entering the horizon in the radiation era. A change $\Delta N_{\nu}=1$ leads to $\Delta C_{l} / C_{l} \approx-0.04$. Since the CMB modes entering in the matter era and not experiencing the suppression are limited to large scales, where sampling variance is large, this effect by itself cannot be extracted with high precision. However, neutrino perturbations amplify the CDM modes entering the horizon in the radiation era. The effect is further enhanced by the fact that while CMB physics is more sensitive to the ratios $\omega_{b} / \omega_{\gamma}$ and $\omega_{m} / \omega_{r}$, specifying the acoustic dynamics and the background evolution, matter fluctuations are also sensitive to the ratio $\omega_{b} / \omega_{m}$, which, if fixing the natural CMB variables, cannot be held fixed under varying $N_{\nu}$. This changes the present matter fluctuation spectrum on small scales by $\Delta P_{m} / P_{m} \approx 0.12$ for $\Delta N_{\nu}=1$, of which 0.04 is due to neutrinos and the rest to $\omega_{b} / \omega_{\gamma}$ variation.

It is unclear how accurately can this effect be extracted from local probes of large scale structure, such as galaxy clustering and weak lensing, since nonlinear evolution will complicate or, in the case of galaxy clustering, prevent its determination on small scales. Weak lensing of CMB traces matter fluctuations on larger scales and higher redshifts than any other method. It may be the optimal tool to use here since nonlinear reconstruction methods using the nongaussian information, especially from polarization data [93, 98], can achieve high signal to noise on the projected convergence power spectrum. However, from the Fisher matrix analysis we find that lensing of CMB cannot improve the limits from primary $\mathrm{CMB}$ and its polarization significantly.

Finally, $N_{\nu}$ variation changes the relativistic energy density and thus changes the relation between the expansion factor and time. This leads to a change in the proper size of the acoustic horizon and so in its angular size, which determines the positions of acoustic peaks. The angular size of the horizon, however, is degenerate with other parameters, such as those changing the angular diameter distance to recombination. The change of the expansion time scales also modifies the recombination process, the visibility function, and the angular damping scale. The effect on the CMB power spectrum can be significant, reaching $15 \%$ power suppression at $l=3000$ for $\Delta N_{\nu}=1$, Fig. 5. However, this can be mimicked by different primordial helium abundance: a change of $\Delta N_{\nu}=0.1$ is compensated by $\Delta Y \simeq-5 \times 10^{-3}$. If CMB data is used to constrain $n_{B} / n_{\gamma}$ at BBN then the standard BBN limits on $Y$ are already at the level of $\Delta Y \lesssim 10^{-3}[11,12]$, suggesting that $Y$ can be assumed fixed. These limits are not applicable in the models where the photon entropy changes between BBN and CMB decoupling or in non-standard BBN models with $\nu / \bar{\nu}$ asymmetries or particle decays.
In summary, the effects of ultra-relativistic neutrinos on CMB and matter power spectrum are generally small. This is why only weak limits on the neutrino background density have been placed from the available observations. On the other hand, neutrinos give rise to unique effects which exist on small scales and are thus less limited by sampling variance. As a consequence, future CMB experiments should be able to improve the limits significantly. While Planck will be able to determine $N_{\nu}$ with a standard deviation 0.24 , or 0.20 if $Y$ is constrained, a dedicated CMB polarization experiment should improve this bound even further, reaching accuracy levels of 0.09 without $Y$ constraint, or 0.05 if $Y$ is constrained. This will allow one to test the details of neutrino decoupling and the scenarios giving rise to a nonstandard number of neutrino species.

\section{Acknowledgments}

SB is grateful to E. Bertschinger, J. R. Bond, and A. Loeb for their encouragement in studying neutrino perturbations, and to C. Lunardini for a discussion of BBN physics. We both thank E. Bertschinger and V. Mukhanov for useful discussions, A. Makarov and P. McDonald for valuable help with numerical calculations, and S. Weinberg for suggestions allowing to extend the superhorizon conservation laws of Sec. II B to the inflationary epoch. SB has been supported by Princeton University Dicke Fellowship; US by Packard Foundation, Sloan Foundation, NASA NAG5-1993 and NSF CAREER-0132953.

\section{APPENDIX A: COSMOLOGICAL DYNAMICS}

Given mutually non-interacting, except than gravitationally, groups of cosmological species $\{a\}$, one can define $^{19}$ for each group an energy-momentum tensor $T_{a}^{\mu \nu}$ that satisfies the local conservation law $T_{a}^{\mu \nu}{ }_{; \nu}=0$. The total energy-momentum tensor

$$
T^{\mu \nu}=\sum_{a} T_{a}^{\mu \nu}
$$

sources space-time curvature perturbations, as described by the Einstein equations $R_{\mu \nu}-\frac{1}{2} g_{\mu \nu} R=8 \pi G T_{\mu \nu}$.

\section{Background}

We study cosmological perturbations relative to a spatially homogeneous and isotropic model with the metric

$$
d s^{2}=a^{2}(\tau)\left(-d \tau^{2}+\gamma_{i j} d x^{i} d x^{j}\right) .
$$

\footnotetext{
19 The definition is $T_{a}^{\mu \nu}(x) \equiv(2 / \sqrt{-g}) \delta S_{a} / \delta g_{\mu \nu}(x)$, where $S_{a}$ are
} the terms of the action that describe the species $a$. 
The spatial part of the background metric may be written as

$$
\gamma_{i j} d x^{i} d x^{j}=\frac{d r^{2}}{1-K r^{2}}+r^{2} d \Omega^{2}
$$

For most applications of this paper, except for the late time evolution of the matter perturbations, the background curvature $K$ can be neglected. In this case, we take $\gamma_{i j}=\delta_{i j}$.

The background expansion rate with respect to the conformal time $\tau$ is denoted by $\mathcal{H} \equiv \dot{a} / a=a H$. By the Friedmann equation, it equals

$$
\mathcal{H}^{2}=\frac{8 \pi G a^{2}}{3} \rho-K,
$$

where $\rho=-T^{0}{ }_{0}$.

In the unperturbed universe,

$$
T_{a 0}^{0}=-\rho_{a}, \quad T_{a i}^{0}=0, \quad T_{a j}^{i}=\delta_{j}^{i} p_{a} .
$$

Assuming that the species pressure $p_{a}$ is uniquely specified locally by the species energy density $\rho_{a}$, we introduce

$$
\begin{aligned}
w_{a} & \equiv \frac{p_{a}}{\rho_{a}}, \\
c_{a}^{2} & \equiv \frac{d p_{a}}{d \rho_{a}}=w_{a}+\frac{d w_{a}}{d \ln \rho_{a}} .
\end{aligned}
$$

For the similar quantities applied to all the cosmological species together, with $\rho \equiv \sum_{a} \rho_{a}$ and $p \equiv \sum_{a} p_{a}$, we have

$$
\begin{aligned}
w & \equiv \frac{p}{\rho}, \quad \frac{1}{1+w}=\sum_{a} \frac{x_{a}}{1+w_{a}}, \\
c_{w}^{2} & \equiv \frac{\dot{p}}{\dot{\rho}}=\left(\frac{\delta p}{\delta \rho}\right)_{\text {adiab }}=\sum_{a} x_{a} c_{a}^{2} .
\end{aligned}
$$

Here

$$
x_{a} \equiv \frac{\rho_{a}+p_{a}}{\rho+p},
$$

are species enthalpy abundances, satisfying $\sum_{a} x_{a}=1$.

It will prove useful to introduce "reduced" enthalpy background densities

$$
\begin{aligned}
\gamma_{a} & \equiv 4 \pi G a^{2}\left(\rho_{a}+p_{a}\right) \\
\gamma & \equiv 4 \pi G a^{2}(\rho+p)=\sum_{a} \gamma_{a} .
\end{aligned}
$$

From definition (A10) and Friedman equation (A4),

$$
\gamma_{a}=x_{a} \gamma, \quad \gamma=3(1+w)\left(\mathcal{H}^{2}+K\right) / 2 .
$$

Finally, we give the rate of change of some of the above quantities with respect to the conformal time $\tau$. Energy conservation requires

$$
\dot{\rho}_{a}=-3 \mathcal{H}\left(\rho_{a}+p_{a}\right),
$$

This and the Friedmann equations give

$$
\begin{aligned}
\dot{\mathcal{H}}=\frac{1}{a} \frac{d^{2} a}{d t^{2}} & =-\frac{4 \pi G a^{2}}{3}(\rho+3 p)= \\
& =-\frac{1+3 w}{2}\left(\mathcal{H}^{2}+K\right) .
\end{aligned}
$$

By eqs. (A9, A14),

$$
\dot{w}=3 \mathcal{H}(1+w)\left(w-c_{w}^{2}\right) .
$$

By eqs. (A7, A9) and (A14),

$$
\dot{\gamma}_{a}=-\mathcal{H}\left(1+3 c_{a}^{2}\right) \gamma_{a}, \quad \dot{\gamma}=-\mathcal{H}\left(1+3 c_{w}^{2}\right) \gamma .
$$

From the last two equations we also see that

$$
\dot{x}_{a}=\left(\frac{\gamma_{a}}{\gamma}\right)^{\cdot}=-3 \mathcal{H}\left(c_{a}^{2}-c_{w}^{2}\right) x_{a} .
$$

\section{Matter perturbations}

We parameterize $T_{a \nu}^{\mu}$ perturbations by the particle number overdensity ${ }^{20} \delta_{a}=\delta n_{a} / n_{a}$, the peculiar velocity vector $v_{a}^{i}$, and the anisotropic stress perturbation $\Pi_{a}^{i j}$ :

$$
\begin{aligned}
& T_{a 0}^{0}=-\left(\rho_{a}+\delta \rho_{a}\right), \quad \delta \rho_{a} \equiv\left(\rho_{a}+p_{a}\right) \delta_{a}, \\
& T_{a i}^{0}=\left(\rho_{a}+p_{a}\right) v_{i a}, \\
& T_{a j}^{i}=\delta_{j}^{i}\left(p_{a}+\delta p_{a}\right)+\left(\rho_{a}+p_{a}\right) \Pi_{a j}^{i}, \quad \Pi_{a i}^{i} \equiv 0 .
\end{aligned}
$$

In scalar modes the 3 -vectors $v_{i a}$ and 3 -tensors $\Pi_{a j}^{i}$ are derivatives of scalar velocity potentials $u_{a}$,

$$
v_{i a}=-\nabla_{i} u_{a}
$$

and anisotropic stress potentials $\pi_{a}$,

$$
\Pi_{a j}^{i}=\frac{3}{2}\left(\nabla^{i} \nabla_{j}-\frac{1}{3} \delta_{j}^{i} \nabla^{2}\right) \pi_{a} .
$$

With the normalization (A21), $\nabla_{i} \nabla_{j} \Pi_{a}^{i j}=\nabla^{4} \pi_{a}$. The potentials $\pi_{a}$ are related to some of the alternative variables used to describe anisotropic stress as $\sigma=-\nabla^{2} \pi$ for the variable of Ref. [57] and $\Pi=-(3 / 2)(1+\rho / p) \nabla^{2} \pi$ for Ref. [60].

For the perturbations in the total energy-momentum tensor $T^{\mu}{ }_{\nu}$, parameterized analogously to eqs. (A19),

$$
\delta=\sum_{a} x_{a} \delta_{a}, \quad u=\sum_{a} x_{a} u_{a}, \quad \pi=\sum_{a} x_{a} \pi_{a},
$$

where $x_{a}$ were defined in eq. (A10).

\footnotetext{
20 Many authors use $\delta_{a}$ for the energy overdensity $\delta \rho_{a} / \rho_{a}=(1+$ $\left.w_{a}\right) \delta_{a}$, in our notations.
} 


\section{Metric and Gauges}

Under a reparameterization of spacetime coordinates (gauge transformation)

$$
\tilde{\tau}=\tau+\delta \tau(\tau, \mathbf{r}), \quad \tilde{x}^{i}=x^{i}+\delta x^{i}(\tau, \mathbf{r}),
$$

the perturbation variables transform as

$$
\tilde{\delta}_{a}=\delta_{a}+3 \mathcal{H} \delta \tau, \quad \tilde{u}_{a}=u_{a}-\delta \tau, \quad \tilde{\pi}_{a}=\pi_{a} .
$$

Note that the following quantities are gauge invariant: $\delta_{a}+3 \mathcal{H} u_{a}$, or $\delta_{a}-\delta_{b}$, or $u_{a}-u_{b}$.

The perturbed metric of space-time can be parameterized as

$$
\begin{aligned}
d s^{2}=a^{2}(\tau)[ & (-1-2 A) d \tau^{2}-2 B_{i} d \tau d x^{i}+ \\
& \left.+\left(1-2 H_{L}\right) d \mathbf{r}^{2}-2 \bar{H}_{i j} d x^{i} d x^{j}\right],
\end{aligned}
$$

where $\bar{H}_{i}^{i} \equiv 0$. For scalar modes, the 3 -vector $B_{i}$ and the 3 -tensor $\bar{H}_{i j}$ can be written as spatial derivatives of scalar functions:

$$
B_{i}=\nabla_{i} b, \quad \bar{H}_{j}^{i}=\left(\nabla^{i} \nabla_{j}-\frac{1}{3} \delta_{j}^{i} \nabla^{2}\right) \chi
$$

The metric perturbations transform under the gauge transformation (A23) as

$$
\begin{aligned}
\tilde{A} & =A-\delta \dot{\tau}-\mathcal{H} \delta \tau \\
\tilde{B}_{i} & =B_{i}-\nabla_{i} \delta \tau+\delta \dot{x}_{i} \\
\tilde{H}_{L} & =H_{L}+\mathcal{H} \delta \tau+\frac{1}{3} \nabla_{i} \delta x^{i}, \\
\tilde{\bar{H}}_{j}^{i} & =\bar{H}_{j}^{i}+\frac{1}{2}\left(\nabla^{i} \delta x_{j}+\nabla_{j} \delta x^{i}\right)-\frac{1}{3} \delta_{j}^{i} \nabla_{k} \delta x^{k}
\end{aligned}
$$

For scalar perturbations with

$$
\delta x_{i} \equiv \nabla_{i} \delta \lambda
$$

the potentials $b$ and $\chi$ in eq. (A26) transform as

$$
\tilde{b}=b-\delta \tau+\delta \dot{\lambda}, \quad \tilde{\chi}=\chi+\delta \lambda
$$

In the conformal Newtonian (longitudinal) gauge the gauge conditions on scalar perturbations are $b^{(N)} \equiv 0$ and $\chi^{(N)} \equiv 0$. For brevity, we refer to this gauge as the "Newtonian". Defining $\Phi \equiv A^{(N)}$ and $\Psi \equiv H_{L}^{(N)}$ we arrive at the metric of eq. (1).

In the synchronous gauge one sets $A^{(s)} \equiv 0$ and $B_{i}^{(s)} \equiv 0$. The observers who are at rest in the synchronous gauge are free falling in the gravitational field and their locally measured proper time sets the coordinate time. By eqs. (A31), the gauge transformation from the synchronous to the Newtonian gauge is $\delta \tau=-\dot{\chi}^{(s)}$, $\delta \lambda=-\chi^{(s)}$. Hence, from eqs. (A27, A29), the Newtonian potentials are related to the scalar metric perturbations in the synchronous gauge as

$$
\Phi=\ddot{\chi}^{(s)}+\mathcal{H} \dot{\chi}^{(s)}, \quad \Psi=H_{L}^{(s)}-\mathcal{H} \dot{\chi}^{(s)}-\frac{1}{3} \nabla^{2} \chi^{(s)} .
$$

For the reverse transformation from the Newtonian to the synchronous gauge, by eqs. (A27, A31), $\delta \tau(x)$ and $\delta \lambda(x)$ are any functions satisfying

$$
\delta \dot{\tau}+\mathcal{H} \delta \tau=\Phi, \quad \delta \dot{\lambda}=\delta \tau
$$

The initial values $\delta \tau\left(\tau_{\text {in }}, \mathbf{r}\right)$ and $\delta \lambda\left(\tau_{\text {in }}, \mathbf{r}\right)$ can be chosen arbitrarily, corresponding to the residual gauge freedom of the synchronous gauge. The metric perturbations in the synchronous gauge are obtained as

$$
H_{L}^{(s)}=\Psi+\mathcal{H} \delta \tau+\frac{1}{3} \nabla^{2} \delta \lambda, \quad \chi^{(s)}=\delta \lambda
$$

In the spatially flat gauge the scalar perturbations of the spatial part of the metric are absent: $H_{L}^{(f)} \equiv 0$ and $\chi^{(f)} \equiv 0$. By eqs. (A29, A31), this gauge has no residual gauge freedom. It is obtained from the Newtonian gauge with $\delta \tau=-\Psi / \mathcal{H}, \delta \lambda=0$. In terms of the Newtonian potentials, the scalar metric perturbations in the spatially flat gauge are

$$
A^{(f)}=\Phi+\Psi+\left(\frac{\Psi}{\mathcal{H}}\right)^{\cdot}, \quad b^{(f)}=\frac{\Psi}{\mathcal{H}} .
$$

The comoving gauge is defined as the gauge in which the scalar components of the total $T_{i}^{0}$ vanishes: $u^{(c)} \equiv$ 0. By eq (A24), the transformation from Newtonian to the comoving gauge is achieved with $\delta \tau=u^{(N)}$. For the second gauge condition, fixing $\delta x^{i}$ in eq. (A23), it is convenient to choose $\chi^{(c)} \equiv 0$. Then $\delta x^{i}=0$. By eq. (A29), the spatial curvature potential in the comoving gauge is related to the Newtonian gauge variables as

$$
\mathcal{R} \equiv H_{L}^{(c)}=\Psi+\mathcal{H} u^{(N)}
$$

For the remaining scalar metric perturbations eqs. (A27, A31) give

$$
A^{(c)}=\Phi-\dot{u}^{(N)}-\mathcal{H} u^{(N)}, \quad b^{(c)}=-u^{(N)} .
$$

By the following eq. (A43) and eq. (A13), the "comoving curvature" $\mathcal{R}$ can be easily transformed into its conventional form

$$
\mathcal{R}=\Psi+\frac{2}{3(1+w)}\left(\Phi+\frac{\dot{\Psi}}{\mathcal{H}}\right)
$$

The uniform density gauge corresponds to the condition $\delta^{(u)} \equiv 0$. Hence, eq (A24), it is obtained from the Newtonian gauge with $\delta \tau=-\delta^{(N)} /(3 \mathcal{H})$. Taking $\chi^{(u)} \equiv 0$ to be the second gauge condition, one finds 
that the 3 -curvature potential $H_{L}$ in the uniform density gauge is

$$
\zeta \equiv H_{L}^{(u)}=\Psi-\frac{1}{3} \delta^{(N)} .
$$

By Einstein equations (A42-A43), the curvature perturbations in the comoving, uniform density, and Newtonian gauges are related as

$$
\mathcal{R}=\zeta+\frac{\nabla^{2} \Psi}{3 \gamma} .
$$

\section{Conservation and Einstein Equations}

\section{Newtonian gauge:}

The dynamics of the species density and velocity perturbations follows from the conservation law $T_{a}^{\mu \nu}{ }_{; \nu}=0$ as

$$
\begin{aligned}
& \dot{\delta}_{a}=\nabla^{2} u_{a}+3 \dot{\Psi}, \\
& \dot{u}_{a}=c_{a}^{2} \delta_{a}-\chi_{a} u_{a}+\nabla^{2} \pi_{a}+\Phi
\end{aligned}
$$

(for the scalar mode), where $\chi_{a} \equiv \mathcal{H}\left(1-3 c_{a}^{2}\right)$ is the Hubble drag rate for the species $a$. The evolution of the anisotropic stress potential $\pi_{a}$ is determined by the internal dynamics of the species.

The linearized Einstein equations in Newtonian gauge, Ref. [57, 99], are easily reduced to

$$
\begin{aligned}
\nabla^{2} \Psi-3 \mathcal{H}(\dot{\Psi}+\mathcal{H} \Phi) & =\gamma \delta, \\
\dot{\Psi}+\mathcal{H} \Phi & =\gamma u, \\
\ddot{\Psi}+\mathcal{H}(2 \dot{\Psi}+\dot{\Phi})-3 w \mathcal{H}^{2} \Phi & =\gamma \frac{\delta p}{\delta \rho} \delta+\gamma \nabla^{2} \pi, \\
\Psi-\Phi & =3 \gamma \pi,
\end{aligned}
$$

where $\gamma$ is introduced by eqs. (A12, A13) and the background is assumed spatially flat. If for all the species $\delta p_{a}=c_{a}^{2} \delta \rho_{a}$ then $(\delta p / \delta \rho) \delta=\sum_{a} x_{a} c_{a}^{2} \delta_{a}$. By equations (A42-A43),

$$
\nabla^{2} \Psi=\gamma \delta^{(c)}
$$

where $\delta^{(c)}=\sum_{a} x_{a} \delta_{a}^{(c)}$ is the averaged particle number density perturbation in the comoving gauge,

$$
\delta_{a}^{(c)}=\delta_{a}+3 \mathcal{H} u
$$

Synchronous gauge:

In Appendix B we refer to the evolution equations in the synchronous gauge. In this gauge the energy and momentum conservation equations give

$$
\begin{aligned}
& \dot{\delta}_{a}^{(s)}=\nabla^{2} u_{a}^{(s)}+3 \dot{H}_{L}^{(s)}, \\
& \dot{u}_{a}^{(s)}=c_{a}^{2} \delta_{a}^{(s)}-\chi_{a} u_{a}^{(s)}+\nabla^{2} \pi_{a} .
\end{aligned}
$$

The corresponding linearized Einstein equations, e.g. Ref. [57], in spatially flat background are

$$
\begin{array}{r}
\nabla^{2}\left(H_{L}^{(s)}-\frac{1}{3} \nabla^{2} \chi^{(s)}\right)-3 \mathcal{H} \dot{H}_{L}^{(s)}=\gamma \delta^{(s)} \\
\dot{H}_{L}^{(s)}-\frac{1}{3} \nabla^{2} \dot{\chi}^{(s)}=\gamma u^{(s)}, \\
\ddot{H}_{L}^{(s)}+\mathcal{H} \dot{H}_{L}^{(s)}=\gamma\left(\frac{\delta p}{\delta \rho}+\frac{1}{3}\right) \delta^{(s)} \\
\ddot{\chi}^{(s)}+2 \mathcal{H} \dot{\chi}^{(s)}-\left(H_{L}^{(s)}-\frac{1}{3} \nabla^{2} \chi^{(s)}\right)=-3 \gamma \pi .
\end{array}
$$

\section{Dynamics in phase space}

Six variables specify the coordinates of a particle in phase space at a given time. For them, following Refs. [3, 57], we take the comoving coordinates of the particle $r^{i}$ and the comoving momenta

$$
q_{i} \equiv a p_{i}
$$

where $p_{i}$ are the proper momenta measured by a comoving observer, who is at rest with respect to the coordinate frame. For a particle with a mass $m_{a}$ the momenta that are canonically conjugate to the variables $r^{i}$ are $P_{i}=m_{a} d x_{i} / \sqrt{-d s^{2}}=(1-\psi) q_{i}$.

The particle density in phase space is specified by the canonical phase space distribution $f_{a}\left(r^{i}, P_{j}, \tau\right)$ :

$$
d N_{a}=f_{a}\left(r^{i}, P_{j}, \tau\right) d^{3} r^{i} d^{3} P_{j}
$$

for every species of particles and their states of polarization $a$. The energy-momentum tensor of the species $a$ is given in the Newtonian gauge by the following simple expression up to the first order of cosmological perturbation theory $[3,57]$ :

$$
T_{a \nu}^{\mu}=\int d^{3} p_{i} \frac{p^{\mu} p_{\nu}}{p^{0}} f_{a}
$$

with $p^{0} \equiv-p_{0} \equiv \sqrt{(q / a)^{2}+m_{a}^{2}}$ and $p^{i} \equiv p_{i}=q_{i} / a$. Below we drop the species label $a$ when referring to any sort of particles in general.

The evolution of the phase space distributions obeys the Boltzmann equation:

$$
\dot{f}+\dot{r}^{i} \frac{\partial f}{\partial r^{i}}+\dot{q} \frac{\partial f}{\partial q}+\dot{n}_{i} \frac{\partial f}{\partial n_{i}}=\left(\frac{\partial f}{\partial \tau}\right)_{C},
$$

where $f$ is considered as a function of the coordinates $r^{i}, q \equiv\left|q_{i}\right|, n_{i} \equiv q_{i} / q$, and $\tau$. The right hand side of 
eq. (A56) describes the change of the phase space density due to particle collisions. Linearizing the Boltzmann equation relative to an unperturbed background phase space distribution, $f=\bar{f}+\delta f$, in the Newtonian gauge one finds, Ref. [57]:

$$
\begin{aligned}
(\delta f)^{\cdot}+\frac{q_{i}}{\epsilon} \nabla_{i}(\delta f)+q \frac{\partial \bar{f}}{\partial q}(\dot{\Psi}- & \left.\frac{\epsilon}{q} n_{i} \nabla_{i} \Phi\right)= \\
& =(\dot{f})_{C}-(\dot{\bar{f}})_{C}
\end{aligned}
$$

where $\epsilon \equiv \sqrt{q^{2}+a^{2} m^{2}}$.

\section{APPENDIX B: LOCALITY OF ADIABATIC GREEN'S FUNCTIONS}

In this Appendix for any "reasonable" cosmological system we construct the perturbation Green's functions in that all the scalar gravitational and matter distribution potentials are identically zero in the Newtonian or synchronous gauges beyond the Green's function particle horizon $|x|>\tau$. We argue that the perturbations formed with these Green's functions by their convolution with any smooth kernel are adiabatic, as defined in footnote 8 on p. 6. A Green's function Fourier component with any wave number $k$ presents a non-zero non-decaying adiabatic mode.

We assume that all the scalar perturbations of matter distributions, classical fields, or the metric tensor in our system can be parameterized by a sum of 3-scalar functions (matter potentials) acted by polynomials of $\nabla_{i}$. This is easily achieved for classical fields that are scalars, vectors, or tensors of a higher integer rank. Complications would arise for spinor and other non-integer spin fields. However, being fermions, such fields can not support coherent semiclassical excitations. Without sacrifice of generality, we require that the polynomials of $\nabla_{i}$ are homogeneous $l=0,1$, etc. degree polynomials that transform under spatial rotations as irreducible tensors of the rank $l$. We treat all the dynamical degrees of freedom, whether they are dynamical "coordinates" or "velocities", on equal footing, implying the Hamiltonian formulation of the system classical dynamics in the coordinate time of the chosen gauge.

We set the Green's function initial conditions at a spatial slice of an infinitesimal thickness at a coordinate time $\tau_{\text {in }}>0$, which is eventually sent to zero. Within this slice we impose the Newtonian gauge conditions $b \equiv 0$ and $\chi \equiv 0$ on the scalar metric potentials (A26). We require that, in the Newtonian gauge, all the matter distribution or field perturbation potentials initially vanish for $|x|>\tau_{\text {in }}$. (The Green's functions considered in this paper are homogeneous in the normal to $x$ spatial directions $y$ and $z$, Sec. IV.) Inside the interval $|x| \leq \tau_{\text {in }}$, to be specific, we set all the $l \geq 1$ matter distribution potentials at $\tau_{\text {in }}$ to zero and adjust the initial conditions for classical fields, if any are present, so that $u\left(\tau_{\text {in }}\right)=0$.
(Then the metric on the initial spatial slice satisfies the comoving gauge condition as well. The spatial slices in the Newtonian and comoving gauges will, in general, differ for $\tau>\tau_{\text {in. }}$ ) At the time $\tau_{\text {in }}$ by eq. (23)

$$
\nabla^{2} \Psi-3 \gamma \Psi=\gamma d
$$

Let us demand that $\Psi\left(\tau_{\text {in }}, x\right)=\left(1 / \tau_{\text {in }}\right) \bar{\Psi}_{0}\left(x / \tau_{\text {in }}\right)$ where $\bar{\Psi}_{0}(\chi)$ is an arbitrarily chosen even function that vanishes for $|\chi|>1$ and that has a non-zero integral over $d \chi$ from -1 to 1 . Then the coordinate "particle number" density perturbation $d\left(\tau_{\text {in }}, x\right)$ should be given by the right hand side of eq. (B1). The corresponding proper number density perturbation $\delta$ at $\tau_{\text {in }}$ is

$$
\delta=d+3 \Psi=\frac{1}{\gamma} \nabla^{2} \Psi
$$

We set all the species initial densities and the initial conditions for classical fields so that $\delta_{a}\left(\tau_{\text {in }}, x\right)=(1 / \gamma) \nabla^{2} \Psi$ and all the other matter potentials are even and vanish for $|x|>\tau_{\text {in }}$. For interacting species, the separation of the total energy-momentum tensor $T^{\mu \nu}$ into $T_{a}^{\mu \nu}$ and so the definition of the species density perturbations $\delta_{a}$ may be ambiguous. In this case, we take any of the possible definitions. In the limit $\tau_{\text {in }} \rightarrow 0$ all of them lead to the physically identical adiabatic Green's functions. If any of the matter potentials or fields remain unfixed, we take them unperturbed at $\tau_{\text {in }}$ for all $x$.

The motivation for these initial conditions is to construct an initially localized almost "pure" curvature perturbation. For any $\tau_{\text {in }}>0$, the curvature perturbation must be generated by some non-zero matter disturbance. The above procedure attempts to restrict the required matter inhomogeneity so that in the limit $\tau_{\text {in }} / \lambda \rightarrow 0$ only curvature appears to be perturbed. Indeed, after the convolution of the constructed Green's function with a primordial fluctuation field $A(x)$ that is smooth on the comoving scales below some $\lambda>0$, the number density perturbation of any species at $\tau_{\text {in }}$ by eq. (B1) tends to

$$
\begin{aligned}
\lim _{\tau_{\mathrm{in}} \rightarrow 0}\langle\delta(x)\rangle & =\lim _{\tau_{\mathrm{in}} \rightarrow 0} \int d x^{\prime} A\left(x^{\prime}\right) \frac{\nabla^{2} \Psi\left(x-x^{\prime}\right)}{\gamma}= \\
& =\left(\int_{-1}^{1} d \chi \bar{\Psi}_{0}(\chi)\right) \lim _{\tau_{\mathrm{in}} \rightarrow 0} \frac{\nabla^{2} A(x)}{\gamma} .
\end{aligned}
$$

The last limit vanishes as $\tau_{\text {in }}^{2} / \lambda^{2}$. Since the other initial matter distributions are chosen unperturbed, they remain unperturbed after the smoothing. However, the $\tau_{\text {in }} \rightarrow 0$ limits of the smoothed comoving curvature perturbation $\mathcal{R}=\Psi+\mathcal{H} u$ as well as the variable $\zeta=-d / 3$ at $\tau_{\text {in }}$ are non-zero:

$$
\lim _{\tau_{\mathrm{in}} \rightarrow 0}\langle\mathcal{R}(x)\rangle=\lim _{\tau_{\mathrm{in}} \rightarrow 0}\langle\zeta(x)\rangle=\left(\int_{-1}^{1} d \chi \bar{\Psi}_{0}(\chi)\right) A(x) .
$$

Given the locality of the Green's function, which is proved next, and the initially vanishing smoothed matter perturbations, we argue at the end of Sec. II B that the 
smoothed perturbations $\zeta$ and $\mathcal{R}$ remain constant while $\tau \ll \lambda$. The Fourier transformation is a convolution with the kernel $A(x)=\exp (-i k x)$. Therefore, it gives nondecaying non-zero curvature perturbation modes (B4), as long as $\int_{-1}^{1} d \chi \bar{\Psi}_{0}$ is chosen different from zero.

The smoothed density perturbation $\langle\delta(x)\rangle$ will remain as small as $O\left(\tau^{2} / \lambda^{2}\right)$ for $\tau_{\text {in }}<\tau \ll \lambda$ in the comoving gauge, where it is always related to $\Psi$ by eq. (A46). In other gauges, including the Newtonian, the proper density perturbation $\langle\delta(x)\rangle$ and other proper matter perturbations do not need to vanish for $\tau_{\text {in }}<\tau \ll \lambda$. For example, the species proper density and velocity perturbations are given by eq. (A24), where $\delta \tau$ is the time lapse between the comoving and the considered gauge.

Now we prove that in a synchronous gauge and the set initial conditions at $\tau_{\text {in }}$ all the matter and gravitational Green's functions remain zero for $\tau>\tau_{\text {in }}$ beyond the particle horizon $|x|>\tau$. The coordinate transformations $\delta \tau$ and $\delta x_{i}=\nabla_{i} \delta \lambda$ from the Newtonian to the synchronous gauge can be chosen so that $\delta \tau\left(\tau_{\text {in }}\right)=0$ and $\delta \lambda\left(\tau_{\text {in }}\right)=0$ for all $x$. Then, from eqs. $(\mathrm{A} 33), \delta \dot{\tau}\left(\tau_{\text {in }}\right)=\Phi\left(\tau_{\text {in }}\right)$ and $\delta \dot{\lambda}\left(\tau_{\text {in }}\right)=0$. Eq. (A34) gives that the synchronous gauge potentials $H_{L}^{(s)}$ and $\chi^{(s)}$ and their first time derivatives vanish at $\tau_{\text {in }}$ for $|x|>\tau_{\text {in }}$. Same is true for the transformed initial perturbations of all the matter distributions and their rates of change.

Causality requires that in the synchronous gauge the matter and the metric remain unperturbed beyond the particle horizon of the original perturbation. This means that $H_{L}^{(s)}, \nabla^{2} \chi^{(s)}$, c.f. eq. (A26), $\nabla u^{(s)}, \nabla^{2} \pi$, etc. are zero for $|x|>\tau$. We argue that the potentials $\chi^{(s)}, u^{(s)}$, $\pi$, etc. themselves are also zero for $|x|>\tau$. As an illustration, let us consider the Euler's equation for $\dot{v}_{x} \equiv-\nabla_{x} \dot{u}$ that in the synchronous gauge reads

$$
\nabla_{x} \dot{u}^{(s)}=\nabla_{x}\left[c_{w}^{2} \delta^{(s)}-\mathcal{H}\left(1-3 c_{w}^{2}\right) u^{(s)}+\nabla^{2} \pi\right] .
$$

The gradient in this and the similar equations can be dropped after we define the expressions under the gradients to be equal at a certain $x_{0}$, which may depend on $\tau$. Consistent definition of the potentials is obtained if $x_{0}$ is chosen outside of the perturbation horizon; suppose, $x_{0}(\tau)<-\tau$. Since a sufficiently high spatial derivative of every synchronous gauge potential gives a perturbation of some matter distribution or a metric tensor component and thus, by above, vanishes for $x<-\tau$, the potentials themselves can be chosen to vanish in that interval for all $\tau$. We prove that the potentials then also vanish for $x>\tau$.

Let $\left\{f_{l}\right\}$ be multipole potentials for some matter distribution perturbation $F(\mathbf{r}, \hat{\mathbf{n}})$, which may depend on other, not displayed, phase space or internal coordinates,

$$
F(\mathbf{r}, \hat{\mathbf{n}}) \equiv \sum_{l=0}^{\infty}(-1)^{l}(2 l+1) P_{l}\left(\frac{n_{i} \nabla_{i}}{\nabla}\right) \nabla^{l} f_{l},
$$

where $i$ runs over the spatial coordinates $x, y$, and $z$. We suppose that the distribution perturbation dynamics can be described as

$$
\dot{F}(\mathbf{r}, \hat{\mathbf{n}})=\left(\begin{array}{c}
\text { local in space functional } \\
\text { of perturbation fields }
\end{array}\right) .
$$

The functional on the right hand side is linear in the linear order of perturbation theory. Multiplying both sides of eq. (B7) by a spherical function $Y_{l m}(\hat{\mathbf{n}})$, integrating over the solid angle $d^{2} \Omega_{\hat{\mathbf{n}}}$, and applying the identity

$$
\int d^{2} \Omega_{\hat{\mathbf{n}}} Y_{l m}(\hat{\mathbf{n}}) P_{l}\left(\frac{n_{i} \nabla_{i}}{\nabla}\right)=\frac{4 \pi}{2 l+1} Y_{l m}\left(\frac{\nabla}{\nabla}\right),
$$

on the left hand side of eq. (B7) we find $\dot{f}_{l}$ times a homogeneous $n$-degree polynomial $Q_{l m}\left(\nabla_{x}, \nabla_{y}, \nabla_{z}\right)$. The polynomials $\left\{Q_{l m}\right\}_{m=-l}^{l}$ transform under spatial rotations as $Y_{l m}$. Since all the perturbation potentials are defined to be 3 -scalars, $\nabla_{i}$ are the only quantities that may appear on the right hand side and that transform non-trivially under spatial rotations. Therefore, after the convolution with $Y_{l m}(\hat{\mathbf{n}})$, every term on the right hand side of the linear equation (B7) must contain the same polynomial $Q_{l m}\left(\nabla_{x}, \nabla_{y}, \nabla_{z}\right)$ as that appears on the left hand side. Putting $m$ to 0 and applying the resulting evolution equation to the $y$ and $z$ independent Green's functions, we find $\left(\nabla_{x}\right)^{l} \dot{f}_{l}$ on the left and at least $l$ derivatives $\nabla_{x}$ in every term on the right hand side. After $l$ integrations over $d x$ with zero initial values at $x<-\tau$ we find an equation of the form

$$
\dot{f}_{l}(\mathbf{r})=\left(\begin{array}{c}
\text { local in space functional } \\
\text { of scalar potentials }
\end{array}\right) \text {. }
$$

The corresponding equations for the studied in the main text system of photon-baryon and CDM fluids and ultra-relativistic neutrinos are eqs. $(4-5,20)$. The scalar potential evolution can be generalized to describe photon-baryon Thompson scattering and photon polarization as shown in the Appendix of Ref. [56]. The evolution of the synchronous metric potentials $H_{L}^{(s)}, \chi^{(s)}$, and $\dot{\chi}^{(s)}$ can as well be presented in the form (B9) using eqs. (A49, A50, A52).

Thus the dynamics of all the potentials in the synchronous gauge can be reduced to an initial value Cauchy's problem. The initial conditions at $\tau=\tau_{\text {in }}$ were chosen even. Assuming the dynamical equations are invariant under $x \rightarrow-x$ inversion, the resulting solution of the Cauchy's problem will remain even for all $\tau$. Hence, the potential values for $x>\tau$ are equal to those at $x<-\tau$, i.e., vanish in the synchronous gauge.

The reverse transformation to the Newtonian gauge is achieved with $\delta \tau=-\dot{\chi}^{(s)}$ and $\delta \lambda=-\chi^{(s)}$, see Sec. A 3 . These functions have been proved to vanish outside of the horizon. Therefore, all the matter multipole potentials in the Newtonian gauge and the gravitational potentials $\Phi$ and $\Psi$, related to the synchronous metric perturbations by eqs. (A32), as well vanish for $|x|>\tau$. 


\section{APPENDIX C: $O\left(R_{\nu}\right)$ ORDER CALCULATIONS IN THE RADIATION ERA}

\section{Gravitational potentials}

In Secs. IV D-IV E we derived integro-differential equations satisfied by the potentials $\Phi_{ \pm} \equiv(\Psi \pm \Phi) / 2$ during radiation domination. We can rewrite these equations, eqs. $(101,100)$ and $(106,105,114-115)$ as

$$
\bar{\Phi}_{-}^{\prime}(\chi)=R_{\nu}\left[\frac{3}{2} \zeta_{\text {in }} \chi\left(1-\chi^{2}\right) \theta(1-|\chi|)-6 \int_{-1}^{\chi} d \chi^{\prime} \int_{-1}^{1} d \chi^{\prime \prime} \frac{\bar{\Phi}_{+}\left(\chi^{\prime \prime}\right) \chi^{\prime} P_{2}\left(\chi^{\prime}\right)+\bar{\Phi}_{+}\left(\chi^{\prime}\right) \chi^{\prime \prime} P_{2}\left(\chi^{\prime \prime}\right)}{\chi^{\prime \prime}-\chi^{\prime}}\right]
$$

and

$$
\bar{\Phi}_{+}(\chi)=\left(\chi^{2}-\frac{1}{3}\right)\left[p_{\Phi} \theta\left(\frac{1}{\sqrt{3}}-|\chi|\right)-F_{-}(\chi)\right]
$$

with

$$
\begin{aligned}
F_{-}(\chi) & =\int_{-1}^{\chi} d \chi^{\prime} \frac{\chi^{\prime 2}-1}{\left(\chi^{\prime 2}-\frac{1}{3}\right)^{2}} \bar{\Phi}_{-}^{\prime}\left(\chi^{\prime}\right) \\
p_{\Phi} & =-\frac{\sqrt{3}\left(1-R_{\nu}\right)}{1-2 R_{\nu}}\left[\frac{3}{2} \zeta_{\text {in }}-\int_{-1}^{1} d \chi F_{-}(\chi)\right]
\end{aligned}
$$

In this Appendix we use them to calculate in $O\left(R_{\nu}\right)$ order the prefactors at the singular terms in the photon and CDM density Green's functions. Up to small $O\left(R_{\nu}^{2}\right)$ corrections, this yields the density modes on subhorizon scales.

The quantity $\bar{\Phi}_{-}^{\prime}$ in $O\left(R_{\nu}\right)$ order is obtained by substituting the zeroth order solution for $\bar{\Phi}_{+}$, eq. (109), into the right hand side of eq. (C1). The substitution gives

$$
\bar{\Phi}_{-}^{\prime}=R_{\nu} \zeta_{\text {in }}\left\{\left[I_{1}(\chi)-I_{0} \operatorname{sign} \chi\right] \theta(1-|\chi|)-\left[I_{2}(\chi)-I_{0} \operatorname{sign} \chi\right] \theta\left(\frac{1}{\sqrt{3}}-|\chi|\right)\right\}+O\left(R_{\nu}^{2}\right)
$$

where

$$
\begin{aligned}
I_{1}(\chi) & \equiv 9 \chi\left(\frac{1}{2} \chi^{4}-\frac{7}{18} \chi^{2}+\frac{1}{9}\right)-\frac{\left(3 \chi^{2}-1\right)^{3}}{4 \sqrt{3}} \ln \left|\frac{\chi+\frac{1}{\sqrt{3}}}{\chi-\frac{1}{\sqrt{3}}}\right| \\
I_{2}(\chi) & \equiv \frac{3 \sqrt{3}}{4}\left(1-\chi^{2}\right)\left[2 \chi\left(3 \chi^{2}+1\right)-\left(3 \chi^{4}+1\right) \ln \left|\frac{1+\chi}{1-\chi}\right|\right] \\
I_{0} & \equiv I_{1}(1)=I_{2}\left(\frac{1}{\sqrt{3}}\right)=2-\frac{2}{\sqrt{3}} \ln (2+\sqrt{3})
\end{aligned}
$$

\section{Photon singularities}

The magnitude of the photon density acoustic spike $p_{\gamma}$, eq. (115), can be calculated analytically using $F_{-}$definition (C3) and the above expression for $\bar{\Phi}_{-}^{\prime}$. For this we employed the symbolic calculation program "Mathematica" (http://www.wolfram.com). The resulting expression turns out rather lengthy but is easily evaluated to

$$
p_{\gamma} \simeq \frac{3}{2} \zeta_{\text {in }}\left[1-0.2683 R_{\nu}+O\left(R_{\nu}^{2}\right)\right] .
$$

The residue $r_{\gamma}$, as defined by eq. (118), follows from eqs. $(112,106)$ as

$$
r_{\gamma}=\bar{\Phi}_{+}\left(\frac{1}{\sqrt{3}}\right)=\zeta_{\text {in }} R_{\nu}\left(\frac{I_{0}}{\sqrt{3}}-\frac{1}{9}\right) \simeq 0.1656 \zeta_{\text {in }} R_{\nu}
$$

Substitution of the found values in eq. (120) leads to the results $(121-122)$.

\section{CDM singularities}

Now we proceed to the calculation of the neutrino corrections $\Delta_{c}$ and $\delta c$ in the CDM density perturbation (128). The equation for the radiation era CDM Green's function (125) gives

$$
\left(\chi \bar{d}_{c}\right)^{\prime}=\frac{1}{\chi} \bar{\Phi}^{\prime}+p_{c 1} \delta_{\mathrm{D}}(\chi) .
$$

Integrating this expression, fixing $p_{c 1}$ by the requirement that $\chi \bar{d}_{c}$ is an odd function that vanishes at $|\chi|>1$, and 
dividing the result by $\chi$, we obtain

$$
\bar{d}_{c}=\left[\frac{G(\chi)}{\chi}-\frac{G(1)}{|\chi|}\right] \theta(1-|\chi|)+p_{c} \delta_{\mathrm{D}}(\chi),
$$

where

$$
G(\chi) \equiv \int_{0}^{\chi} \frac{d \tilde{\chi} \bar{\Phi}^{\prime}(\tilde{\chi})}{\tilde{\chi}}
$$

The initial condition $\int_{-1}^{1} d \chi \bar{d}_{c}=-3 \zeta_{\text {in }}$ fixes the new constant $p_{c}$ as

$$
p_{c}=-3 \zeta_{\text {in }}-\int_{-1}^{1} d \chi\left[\frac{G(\chi)}{\chi}-\frac{G(1)}{|\chi|}\right] .
$$

The $d \chi$ integral of $G(1) /|\chi|$, which would be infinite in Riemann sense, is equal to 0 in the sense of generalized function integration, see Table I on p. 15. Hence,

$$
p_{c}=-3 \zeta_{\text {in }}-\int_{-1}^{1} \frac{d \chi G(\chi)}{\chi} .
$$

The CDM density perturbation modes in the radiation era are given by the Fourier components of the Green's function (C9). In the radiation era for adiabatic initial conditions the potential $\bar{\Phi}(\chi)$, generated by the photon and neutrino perturbations, is regular at $\chi=0$ and even. Hence, the function $G(\chi)$ in eq. (C10) is regular at $\chi=0$ and odd, and so is $G(\chi) / \chi$ regular at the origin. The subhorizon $(k \tau \gg 1)$ values of the CDM Fourier modes are fully specified by the singular terms in eq. (C9), which are proportional to $1 /|\chi|$ and $\delta_{\mathrm{D}}(\chi)$. From Table II on p. 16 we find that

$$
d_{c}(\tau, k)=A_{c}\left(\ln \varphi_{s}+c\right)+O\left(\varphi_{s}^{-1}\right),
$$

with $\varphi_{s}=k \tau / \sqrt{3}$ and

$$
A_{c}=2 G(1), \quad c=\gamma+\frac{\ln 3}{2}+\frac{p_{c}}{2 G(1)} .
$$

In the $R_{\nu} \rightarrow 0$ limit, with the potentials (108-109),

$$
G^{\left(R_{\nu} \rightarrow 0\right)}(\chi)=-3 \zeta_{\text {in }}\left\{\begin{array}{cc}
\chi \sqrt{3}, & |\chi| \leq \frac{1}{\sqrt{3}} \\
\operatorname{sign} \chi, & |\chi| \geq \frac{1}{\sqrt{3}}
\end{array}\right.
$$

Then $G^{\left(R_{\nu} \rightarrow 0\right)}(1)=-3 \zeta_{\text {in }}$ and

$$
\begin{aligned}
& \bar{d}_{c}^{\left(R_{\nu} \rightarrow 0\right)}= \\
& =-3 \zeta_{\text {in }}\left(\sqrt{3}-\frac{1}{|\chi|}\right) \theta\left(\frac{1}{\sqrt{3}}-|\chi|\right)+p_{c} \delta_{\mathrm{D}}(\chi),
\end{aligned}
$$

where by eq. (C11)

$$
p_{c}^{\left(R_{\nu} \rightarrow 0\right)}=3 \zeta_{\text {in }}(1+\ln 3) .
$$

Fourier transforming eq. (C15-C16), where the singular function $1 /|\chi|$ can be transformed using Table II, we obtain the CDM density perturbation mode (127). Of course, the radiation era, $R_{\nu} \rightarrow 0$ Fourier mode could be obtained directly in $k$-space by integrating the evolution equation (124). Now we consider how the CDM density perturbation changes when neutrinos are added.

Although the analytical calculation of the integrals in eqs. $(\mathrm{C} 10, \mathrm{C} 11)$ in the $O\left(R_{\nu}\right)$ order may be possible, it does not appear easy. On the other hand the numerical evaluation of the absolutely convergent integrals in eqs. $(\mathrm{C} 10, \mathrm{C} 11)$, given the potentials $(\mathrm{C} 5,106)$, is straightforward and yields

$$
\begin{aligned}
G(1) & \simeq-3 \zeta_{\text {in }}\left[1+0.2297 R_{\nu}+O\left(R_{\nu}^{2}\right)\right] \\
p_{c} & \simeq 3 \zeta_{\text {in }}\left[1+\ln 3+1.746 R_{\nu}+O\left(R_{\nu}^{2}\right)\right] .
\end{aligned}
$$

Then for $A_{c}$ and $c$, eqs. (C12-C13),

$$
\begin{aligned}
A_{c} & \simeq-6 \zeta_{\text {in }}\left[1+0.2297 R_{\nu}+O\left(R_{\nu}^{2}\right)\right], \\
c & \simeq \gamma-\frac{1}{2}-0.6323 R_{\nu}+O\left(R_{\nu}^{2}\right) .
\end{aligned}
$$

The corresponding values for $\Delta_{c}=A_{c} / A_{c}^{\left(R_{\nu} \rightarrow 0\right)}-1$ and $\delta c=c-c^{\left(R_{\nu} \rightarrow 0\right)}$ are used in Sec. IV F, eq. (129).
[1] E. W. Kolb and M. S. Turner, The Early Universe (Frontiers in Physics, Reading, MA: Addison-Wesley, 1990).

[2] W. Hu and N. Sugiyama, Astrophys. J. 471, 542 (1996).

[3] J. R. Bond and A. S. Szalay, Astrophys. J. 274, 443 (1983).

[4] J. M. Stewart, Astrophys. J. 176, 323 (1972).

[5] P. J. E. Peebles, Astrophys. J. 180, 1 (1973).

[6] Y. I. Izotov and T. X. Thuan, Astrophys. J. 500, 188 (1998).
[7] Y. I. Izotov, T. X. Thuan, and V. A. Lipovetsky, Astrophys. J. Suppl. 108, 1 (1997).

[8] K. A. Olive and G. Steigman, Astrophys. J. Suppl. 97, 49 (1995).

[9] K. A. Olive, G. Steigman, and E. D. Skillman, Astrophys. J. 483, 788 (1997).

[10] A. Peimbert, M. Peimbert, and V. Luridiana, Astrophys. J. 565, 668 (2002).

[11] V. Barger, J. P. Kneller, H. Lee, D. Marfatia, and G. Steigman, Physics Letters B 566, 8 (2003). 
[12] S. Hannestad, Journal of Cosmology and Astro-Particle Physics 5, 4 (2003).

[13] D. N. Spergel et al., Astrophys. J. Suppl. 148, 175 (2003).

[14] A. D. Dolgov et al., Nuclear Physics B 632, 363 (2002).

[15] K. N. Abazajian, J. F. Beacom, and N. F. Bell, Phys. Rev. D 66, 13008 (2002).

[16] C. Lunardini and A. Y. Smirnov, Phys. Rev. D 64, 73006 (2001).

[17] Y. Y. Y. Wong, Phys. Rev. D66, 025015 (2002).

[18] V. Barger, J. P. Kneller, P. Langacker, D. Marfatia, and G. Steigman, Physics Letters B 569, 123 (2003).

[19] M. Tegmark et al., astro-ph/0310723.

[20] L. Danese and G. De Zotti, Astron. Astrophys. 17, 226 (1982).

[21] E. L. Wright et al., Astrophys. J. 420, 450 (1994).

[22] W. Hu and J. Silk, Phys. Rev. D 48, 485 (1993).

[23] J. C. Mather et al., Astrophys. J. 420, 439 (1994).

[24] A. D. Dolgov, Phys. Reports 370, 333 (2002).

[25] J. Ellis, G. B. Gelmini, J. L. Lopez, D. V. Nanopoulos, and S. Sarkar, Nuclear Physics B 373, 399 (1992).

[26] D. A. Dicus, E. W. Kolb, and V. L. Teplitz, Astrophys. J. 221, 327 (1978).

[27] P. Adhya, D. R. Chaudhuri, and S. Hannestad, Phys. Rev. D 68, 83519 (2003).

[28] M. Kaplinghat and M. S. Turner, Physical Review Letters 86, 385 (2001).

[29] M. Kawasaki, K. Kohri, and N. Sugiyama, Physical Review Letters 82, 4168 (1999).

[30] M. Kawasaki, K. Kohri, and N. Sugiyama, Phys. Rev. D62, 023506 (2000).

[31] D. H. Lyth and E. D. Stewart, Phys. Rev. Lett. 75, 201 (1995).

[32] L. Randall and S. Thomas, Nucl. Phys. B449, 229 (1995).

[33] J. G. Bartlett and L. J. Hall, Physical Review Letters 66, 541 (1991).

[34] R. Bowen, S. H. Hansen, A. Melchiorri, J. Silk, and R. Trotta, MNRAS 334, 760 (2002).

[35] P. Crotty, J. Lesgourgues, and S. Pastor, Phys. Rev. D 67, 123005 (2003).

[36] R. E. Lopez, S. Dodelson, A. Heckler, and M. S. Turner, Physical Review Letters 82, 3952 (1999).

[37] M. Orito, T. Kajino, G. J. Mathews, and Y. Wang, Phys. Rev. D 65, 123504 (2002).

[38] E. Pierpaoli, MNRAS 342, L63 (2003).

[39] S. H. Hansen et al., Phys. Rev. D 65, 23511 (2002).

[40] A. Lewis and S. Bridle, Phys. Rev. D66, 103511 (2002).

[41] A. Lewis, A. Challinor, and A. Lasenby, Astrophys. J. 538, 473 (2000).

[42] U. Seljak and M. Zaldarriaga, Astrophys. J. 469, 437 (1996).

[43] W. Hu, Astrophys. J. 506, 485 (1998).

[44] W. Hu, D. J. Eisenstein, M. Tegmark, and M. White, Phys. Rev. D 59, 23512 (1999).

[45] W. Hu and N. Sugiyama, Astrophys. J. 444, 489 (1995).

[46] C. L. Bennett et al., Astrophys. J. Suppl. 148, 1 (2003).

[47] S. Weinberg, astro-ph/0306304.

[48] L. F. Abbott and R. K. Schaefer, Astrophys. J. 308, 546 (1986).

[49] W. Hu, U. Seljak, M. White, and M. Zaldarriaga, Phys. Rev. D 57, 3290 (1998).

[50] M. Zaldarriaga and U. Seljak, Phys. Rev. D 55, 1830 (1997).

[51] J. C. R. Magueijo, Phys. Rev. D46, 3360 (1992).
[52] C. Baccigalupi, Phys. Rev. D 59, 123004 (1999).

[53] C. Baccigalupi and F. Perrotta, MNRAS 314, 1 (2000).

[54] S. Bashinsky, Ph.D. thesis, MIT (2001).

[55] S. Bashinsky and E. Bertschinger, Physical Review Letters 87, 81301 (2001).

[56] S. Bashinsky and E. Bertschinger, Phys. Rev. D 65, 123008 (2002).

[57] C. Ma and E. Bertschinger, Astrophys. J. 455, 7 (1995).

[58] V. F. Mukhanov, H. A. Feldman, and R. H. Brandenberger, Phys. Rept. 215, 203 (1992).

[59] A. R. Liddle and D. H. Lyth, Cosmological inflation and large-scale structure (Cambridge, UK: Univ. Pr., 2000).

[60] H. Kodama and M. Sasaki, Prog. Theor. Phys. Suppl. 78, 1 (1984).

[61] D. Wands, K. A. Malik, D. H. Lyth, and A. R. Liddle, Phys. Rev. D 62, 43527 (2000).

[62] R. W. Lindquist, Annals Phys. (NY) 37, 487 (1966).

[63] J. M. Bardeen, P. J. Steinhardt, and M. S. Turner, Phys. Rev. D 28, 679 (1983).

[64] Thanks to S. Weinberg for pointing this out.

[65] S. Weinberg, Phys. Rev. D 67, 123504 (2003).

[66] J. M. Bardeen, Phys. Rev. D 22, 1882 (1980).

[67] D. H. Lyth, Phys. Rev. D 31, 1792 (1985).

[68] S. Mollerach, Phys. Rev. D 42, 313 (1990).

[69] D. H. Lyth and D. Wands, Physics Letters B 524, 5 (2002).

[70] C. Gordon and K. A. Malik (2003), astro-ph/0311102.

[71] R. R. Caldwell et al., Astrophys. J. 591, L75 (2003).

[72] G. Dvali and M. S. Turner, astro-ph/0301510.

[73] K. Freese and M. Lewis, Physics Letters B 540, 1 (2002).

[74] J. C. Mather et al., Astrophys. J. 512, 511 (1999).

[75] D. A. Dicus et al., Phys. Rev. D 26, 2694 (1982).

[76] A. F. Heckler, Phys. Rev. D 49, 611 (1994).

[77] R. E. Lopez and M. S. Turner, Phys. Rev. D 59, 103502 (1999).

[78] G. Steigman, astro-ph/0108148.

[79] R. K. Sachs and A. M. Wolfe, Astrophys. J. 147, 73 (1967).

[80] D. S. Jones, The Theory of Generalized Functions (Cambridge University Press, 1982), 2nd ed.

[81] N. Kaiser, MNRAS 202, 1169 (1983).

[82] S. Weinberg, Astrophys. J. 581, 810 (2002).

[83] U. Seljak and M. Zaldarriaga, Physical Review Letters 78, 2054 (1997).

[84] S. Bashinsky, Lecture 8 at http://feynman.princeton.edu/ uros/phy564.html.

[85] L. Page et al., Astrophys. J. Suppl. 148, 233 (2003).

[86] P. J. E. Peebles, Astrophys. J. 153, 1 (1968).

[87] C. Hirata, Lecture 9 at http://feynman.princeton.edu/ uros/phy564.html.

[88] G. Steigman, astro-ph/0308511.

[89] S. Sarkar, Reports of Progress in Physics 59, 1493 (1996).

[90] T. P. Walker, G. Steigman, H. Kang, D. M. Schramm, and K. A. Olive, Astrophys. J. 376, 51 (1991).

[91] M. Zaldarriaga, R. Scoccimarro, and L. Hui, Astrophys. J. 590, 1 (2003).

[92] U. Seljak, Astrophys. J. 482, 6 (1997).

[93] C. M. Hirata and U. Seljak, Phys. Rev. D 68, 83002 (2003).

[94] M. Kaplinghat, L. Knox, and Y.-S. Song, Phys. Rev. Lett. 91, 241301 (2003).

[95] E. Lifshitz, J. Phys. USSR 10, 116 (1946).

[96] P. J. E. Peebles and J. T. Yu, Astrophys. J. 162, 815 (1970). 
[97] S. Xiang and T. Kiang, MNRAS 259, 761 (1992).

[98] W. Hu and T. Okamoto, Astrophys. J. 574, 566 (2002).

[99] E. Bertschinger, in Proc. Les Houches School, Session
LX, ed. R. Schaeffer et al. (Netherlands: Elsevier 1996), astro-ph/9503125. 